\title{
SINGULAR LAGRANGIAN SYSTEMS AND VARIATIONAL CONSTRAINED MECHANICS ON LIE ALGEBROIDS
}

\author{
D. IGLESIAS, J. C. MARRERO, D. MARTÍN DE DIEGO, AND D. SOSA
}

\begin{abstract}
The purpose of this paper is describe Lagrangian Mechanics for constrained systems on Lie algebroids, a natural framework which covers a wide range of situations (systems on Lie groups, quotients by the action of a Lie group, standard tangent bundles...). In particular, we are interested in two cases: singular Lagrangian systems and vakonomic mechanics (variational constrained mechanics). Several examples illustrate the interest of these developments.
\end{abstract}

\section{Contents}

1. Introduction

2. Lie algebroids

2.1. The prolongation of a Lie algebroid over a fibration

2.2. Lagrangian and Hamiltonian mechanics on Lie algebroids

3. Constraint algorithm and reduction for presymplectic Lie algebroids

3.1. Constraint algorithm for presymplectic Lie algebroids

3.2. Reduction of presymplectic Lie algebroids

4. Singular Lagrangian systems on Lie algebroids

5. Vakonomic mechanics on Lie algebroids

5.1. Vakonomic equations and vakonomic bracket

5.2. The variational point of view

5.3. Examples

6. Conclusions and future work

Acknowledgments

References

\section{INTRODUCTION}

There is a vast literature around the Lagrangian formalism in mechanics justified by the central role played by these systems in the foundations of modern mathematics and physics. In some interesting systems some problems often arise due to their singular nature that give rise to the presence of constraints manifesting that the evolution problem is not well posed (internal constraints). Constraints can also

\footnotetext{
2000 Mathematics Subject Classification. 17B66, 37J60, 70F25, 70H30, 70H33, 70G45.

Key words and phrases. Lie algebroids, Lagrangian Mechanics, Hamiltonian Mechanics, constraint algorithm, reduction, singular Lagrangian systems, vakonomic mechanics, variational
} calculus. 
manifest a priori restrictions on the states of the system which are often imposed either by physical arguments or by external conditions (external constraints). Both cases are of considerable importance.

Systems with internal constraints are quite interesting since many dynamical systems are given in terms of non-symplectic forms instead of the more habitual symplectic ones. The more frequent case appears in the Lagrangian formalism of singular mechanical systems which are a commonplace in many physical theories (as in Yang-Mills theories, gravitation, etc). Also, in systems that appears as a limit (as in Chern-Simons lagrangians); for instance consider the following lagrangian

$$
L=\frac{1}{2} m_{i}\left(\dot{q}^{i}\right)^{2}+e A_{i}(q) \dot{q}^{i}-V(q)
$$

in the limit $m_{i} \rightarrow 0$ for some $i$. In other cases, it is necessary work with a new singular lagrangian in a extended space since the original lagrangian is "ill defined" (only locally defined on the original space), as it happens for the electron monopole system (see [16]).

Another motivation for the present work is the study of lagrangian systems subjected to external constraints (holonomic and nonholonomic) 3]. These systems have a wide application in many different areas: engineering, optimal control theory, mathematical economics (growth economic theory), subriemannian geometry, motion of microorganisms, etc.

Constrained variational calculus have a rich geometric structure. Many of these systems usually exhibit invariance under the action of a Lie group of symmetries and they can be notably simplified using their symmetric properties by reducing the degrees of freedom of the original system. In previous studies it is imposed a separate study for each class of systems since the lack of a unified framework for dealing simultaneously with all the systems.

Recent investigations have lead to a unifying geometric framework to covering these plethora of particular situations. It is precisely the underlying structure of a Lie algebroid on the phase space which allows a unified treatment. This idea was first introduced by Weinstein 34] in order to define a Lagrangian formalism which is general enough to account for the various types of systems. The geometry and dynamics on Lie algebroids have been extensively studied during the past years. In particular, in 27, E. Martínez developed a geometric formalism of mechanics on Lie algebroids similar to Klein's formalism of the ordinary Lagrangian mechanics and, more recently, a description of the Hamiltonian dynamics on a Lie algebroid was given in 23 , 28. The key concept in this theory is the prolongation, $\mathcal{T}^{E} E$, of the Lie algebroid over the fibred projection $\tau$ (for the Lagrangian formalism) and the prolongation, $\mathcal{T}^{E} E^{*}$, over the dual fibred projection $\tau^{*}: E^{*} \rightarrow Q$ (for the Hamiltonian formalism). See [23] for more details. Of course, when the Lie algebroid is $E=T Q$ we obtain that $\mathcal{T}^{E} E=T(T Q)$ and $\mathcal{T}^{E} E^{*}=T\left(T^{*} Q\right)$, recovering the classical case. Another approach to the theory was discussed in [14. The existence of symmetries in these systems makes interesting to generalize the Gotay-Nester-Hinds algorithm 13. to the case of Lie algebroids with a presymplectic section. These results are easily extended to the case of implicit differential equations on Lie algebroids.

The second author and collaborators analyzed the case of nonholonomic mechanics on Lie algebroids [8. Now, we also pretend to study singular Lagrangian systems and vakonomic mechanics on Lie algebroids (obtained through the application of a constrained variational principle).

The paper is organized as follows. In Section 2, we recall the notion of a Lie algebroid and several aspects related with it. In particular, we describe the prolongation $\mathcal{T}^{E} E$ of a Lie algebroid $E$ over the projection $\tau: E \rightarrow Q$ and how to 
use this construction to develop Lagrangian mechanics on a Lie algebroid $E$ with a Lagrangian function $L: E \rightarrow \mathbb{R}$, introducing several important objects, such as the Lagrangian energy $E_{L}$ and the Cartan 2-section $\omega_{L}$. When the Lagrangian function is regular (that is, $\omega_{L}$ is nondegenerate), we have existence and uniqueness of solutions for the Euler-Lagrange equations. However, if the Lagrangian is singular (or degenerate) we cannot guarantee these results. Motivated by this fact, in Section 3 we introduce a constraint algorithm for presymplectic Lie algebroids which generalizes the well-known Gotay-Nester-Hinds algorithm. In addition, we show that a Lie algebroid morphism which relates two presymplectic Lie algebroids induces a relation between the two associated constraint algorithms.

In Section 4, we apply the results of Section 3 to singular Lagrangian systems on Lie algebroids. More precisely, given a Lie algebroid $\tau: E \rightarrow Q$ and a singular Lagrangian function $L: E \rightarrow \mathbb{R}$, we look for a solution $X$ of the presymplectic system $\left(\mathcal{T}^{E} E, \omega_{L}, d^{\mathcal{T}^{E}} E E_{L}\right)$ which is a SODE along the final constraint submanifold. An example for an Atiyah algebroid illustrates our theory.

In Section 5, we develop a geometric description of vakonomic mechanics on Lie algebroids. In this setting, given a Lie algebroid $\tau: E \rightarrow Q$, we have a pair $(L, M)$ where $L$ is a Lagrangian function on $E$ and $M \subseteq E$ is a constraint submanifold. In Section 5.1, we deduce the vakonomic equations using our constraint algorithm and study the particular case when it stops in the first step. In this situation, if the restriction of the presymplectic 2-section to the final constraint algebroid is symplectic, one can introduce the vakonomic bracket, which allows us to give the evolution of the observables. On the other hand, it is well know that classical vakonomic systems can be obtained from a constrained variational principle. This can also be done for vakonomic systems on Lie algebroids, as it is shown in Section 5.2. In the particular case when we do not have constraints, our approach can be seen as the Skinner-Rusk formulation of Lagrangian Mechanics on Lie algebroids. This is explained in Section 5.3. where we also illustrate our results with several interesting examples: If $E$ is the standard Lie algebroid $\tau_{T Q}: T Q \rightarrow Q$, then we recover some well-known results (see [9]) for vakonomic systems; in the case when $E=\mathfrak{g}$, a real Lie algebra of finite dimension, we are able to model a certain class of Optimal Control problems on Lie groups (see [21, 22]); for the Atiyah algebroid, we analyze some problems related with reduction in subriemannian geometry by means of the non-holonomic connection 4. Finally, we study Optimal Control on Lie algebroids as vakonomic systems and, as an illustration of our techniques, we find the equations of motion of the Plate-Ball system.

\section{LIE ALGEBROIDS}

Let $E$ be a vector bundle of rank $n$ over a manifold $Q$ of dimension $m$ and $\tau: E \rightarrow Q$ be the vector bundle projection. Denote by $\Gamma(E)$ the $C^{\infty}(Q)$-module of sections of $\tau: E \rightarrow Q$. A Lie algebroid structure $(\llbracket \cdot, \cdot \rrbracket, \rho)$ on $E$ is a Lie bracket $\llbracket \cdot, \cdot \rrbracket$ on the space $\Gamma(E)$ and a bundle map $\rho: E \rightarrow T Q$, called the anchor map, such that if we also denote by $\rho: \Gamma(E) \rightarrow \mathfrak{X}(Q)$ the homomorphism of $C^{\infty}(Q)$-modules induced by the anchor map, then

$$
\llbracket X, f Y \rrbracket=f \llbracket X, Y \rrbracket+\rho(X)(f) Y,
$$

for $X, Y \in \Gamma(E)$ and $f \in C^{\infty}(Q)$. The triple $(E, \llbracket \cdot, \cdot \rrbracket, \rho)$ is called a Lie algebroid over $Q$ (see [25]).

If $(E, \llbracket \cdot, \cdot \rrbracket, \rho)$ is a Lie algebroid over $Q$, then the anchor map $\rho: \Gamma(E) \rightarrow \mathfrak{X}(Q)$ is a homomorphism between the Lie algebras $(\Gamma(E), \llbracket \cdot, \cdot \rrbracket)$ and $(\mathfrak{X}(Q),[\cdot, \cdot])$. 
Standard examples of Lie algebroids are real Lie algebras of finite dimension and the tangent bundle $T Q$ of an arbitrary manifold $Q$.

Another example of a Lie algebroid may be constructed as follows. Let $\pi: P \rightarrow Q$ be a principal bundle with structural group $G$. Denote by $\Phi: G \times P \rightarrow P$ the free action of $G$ on $P$ and by $T \Phi: G \times T P \rightarrow T P$ the tangent action of $G$ on $T P$. Then, one may consider the quotient vector bundle $\tau_{P} \mid G: T P / G \rightarrow Q=P / G$ and the sections of this vector bundle may be identified with the vector fields on $P$ which are invariant under the action $\Phi$. Using that every $G$-invariant vector field on $P$ is $\pi$-projectable and the fact that the standard Lie bracket on vector fields is closed with respect to $G$-invariant vector fields, we can induce a Lie algebroid structure on $T P / G$. The resultant Lie algebroid is called the Atiyah (gauge) algebroid associated with the principal bundle $\pi: P \rightarrow Q$ (see [23, 25]).

If $(E, \llbracket \cdot, \cdot \rrbracket, \rho)$ is a Lie algebroid, one may define the differential of $E, d^{E}$ : $\Gamma\left(\wedge^{k} E^{*}\right) \rightarrow \Gamma\left(\wedge^{k+1} E^{*}\right)$, as follows

$$
\begin{aligned}
d^{E} \mu\left(X_{0}, \ldots, X_{k}\right) & =\sum_{i=0}^{k}(-1)^{i} \rho\left(X_{i}\right)\left(\mu\left(X_{0}, \ldots, \widehat{X}_{i}, \ldots, X_{k}\right)\right) \\
& +\sum_{i<j}(-1)^{i+j} \mu\left(\llbracket X_{i}, X_{j} \rrbracket, X_{0}, \ldots, \widehat{X}_{i}, \ldots, \widehat{X}_{j}, \ldots, X_{k}\right),
\end{aligned}
$$

for $\mu \in \Gamma\left(\wedge^{k} E^{*}\right)$ and $X_{0}, \ldots, X_{k} \in \Gamma(E)$. It follows that $\left(d^{E}\right)^{2}=0$. Moreover, if $X \in \Gamma(E)$, one may introduce, in a natural way, the Lie derivative with respect to $X$, as the operator $\mathcal{L}_{X}^{E}: \Gamma\left(\wedge^{k} E^{*}\right) \rightarrow \Gamma\left(\wedge^{k} E^{*}\right)$ given by $\mathcal{L}_{X}^{E}=i_{X} \circ d^{E}+d^{E} \circ i_{X}$.

Note that if $E=T Q$ and $X \in \Gamma(E)=\mathfrak{X}(Q)$ then $d^{T Q}$ and $\mathcal{L}_{X}^{T Q}$ are the usual differential and the usual Lie derivative with respect to $X$, respectively.

If we take local coordinates $\left(x^{i}\right)$ on $Q$ and a local basis $\left\{e_{A}\right\}$ of sections of $E$, then we have the corresponding local coordinates $\left(x^{i}, y^{A}\right)$ on $E$, where $y^{A}(e)$ is the $A$-th coordinate of $e \in E$ in the given basis. Such coordinates determine local functions $\rho_{A}^{i}, \mathcal{C}_{A B}^{C}$ on $Q$ which contain the local information of the Lie algebroid structure and, accordingly, they are called the structure functions of the Lie algebroid. They are given by

$$
\rho\left(e_{A}\right)=\rho_{A}^{i} \frac{\partial}{\partial x^{i}} \quad \text { and } \quad \llbracket e_{A}, e_{B} \rrbracket=\mathcal{C}_{A B}^{C} e_{C} .
$$

These functions should satisfy the relations

$$
\begin{gathered}
\rho_{A}^{j} \frac{\partial \rho_{B}^{i}}{\partial x^{j}}-\rho_{B}^{j} \frac{\partial \rho_{A}^{i}}{\partial x^{j}}=\rho_{C}^{i} \mathcal{C}_{A B}^{C}, \\
\sum_{\operatorname{cyclic}(A, B, C)}\left(\rho_{A}^{i} \frac{\partial \mathcal{C}_{B C}^{D}}{\partial x^{i}}+\mathcal{C}_{A F}^{D} \mathcal{C}_{B C}^{F}\right)=0,
\end{gathered}
$$

which are usually called the structure equations.

If $f \in C^{\infty}(Q)$, we have that

$$
d^{E} f=\frac{\partial f}{\partial x^{i}} \rho_{A}^{i} e^{A}
$$

where $\left\{e^{A}\right\}$ is the dual basis of $\left\{e_{A}\right\}$. On the other hand, if $\theta \in \Gamma\left(E^{*}\right)$ and $\theta=\theta_{C} e^{C}$ it follows that

In particular,

$$
d^{E} \theta=\left(\frac{\partial \theta_{C}}{\partial x^{i}} \rho_{B}^{i}-\frac{1}{2} \theta_{A} \mathcal{C}_{B C}^{A}\right) e^{B} \wedge e^{C} .
$$

$$
d^{E} x^{i}=\rho_{A}^{i} e^{A}, \quad d^{E} e^{A}=-\frac{1}{2} \varrho_{B C}^{A} e^{B} \wedge e^{C} .
$$


On the other hand, if $(E, \llbracket \cdot, \cdot \rrbracket, \rho)$ and $\left(E^{\prime}, \llbracket \cdot, \cdot \rrbracket^{\prime}, \rho^{\prime}\right)$ are Lie algebroids over $Q$ and $Q^{\prime}$, respectively, then a morphism of vector bundles $(F, f)$ from $E$ to $E^{\prime}$

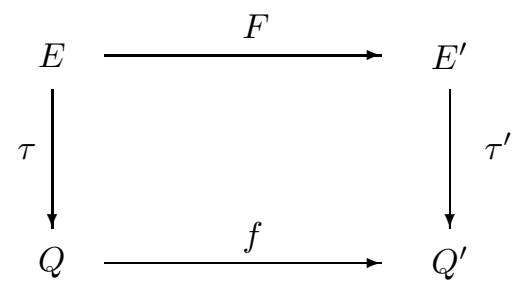

is a Lie algebroid morphism if

$$
d^{E}\left((F, f)^{*} \phi^{\prime}\right)=(F, f)^{*}\left(d^{E^{\prime}} \phi^{\prime}\right), \quad \text { for } \phi^{\prime} \in \Gamma\left(\wedge^{k}\left(E^{\prime}\right)^{*}\right) .
$$

Note that $(F, f)^{*} \phi^{\prime}$ is the section of the vector bundle $\wedge^{k} E^{*} \rightarrow Q$ defined by

$$
\left((F, f)^{*} \phi^{\prime}\right)_{x}\left(a_{1}, \ldots, a_{k}\right)=\phi_{f(x)}^{\prime}\left(F\left(a_{1}\right), \ldots, F\left(a_{k}\right)\right),
$$

for $x \in Q$ and $a_{1}, \ldots, a_{k} \in E_{x}$. We remark that (2.1) holds if and only if

$$
\begin{aligned}
& d^{E}\left(g^{\prime} \circ f\right)=(F, f)^{*}\left(d^{E^{\prime}} g^{\prime}\right), \quad \text { for } g^{\prime} \in C^{\infty}\left(Q^{\prime}\right), \\
& d^{E}\left((F, f)^{*} \alpha^{\prime}\right)=(F, f)^{*}\left(d^{E^{\prime}} \alpha^{\prime}\right), \quad \text { for } \alpha^{\prime} \in \Gamma\left(\left(E^{\prime}\right)^{*}\right) .
\end{aligned}
$$

If $(F, f)$ is a Lie algebroid morphism, $f$ is an injective immersion and $F_{\mid E_{x}}$ : $E_{x} \rightarrow E_{f(x)}^{\prime}$ is injective, for all $x \in Q$, then $\left(E, \llbracket \cdot, \cdot \rrbracket_{E}, \rho_{E}\right)$ is said to be a Lie subalgebroid of $\left(E^{\prime}, \llbracket \cdot, \cdot \rrbracket_{E^{\prime}}, \rho_{E^{\prime}}\right)$.

If $Q=Q^{\prime}$ and $f=i d: Q \rightarrow Q$ then, it is easy prove that the pair $(F, i d)$ is a Lie algebroid morphism if and only if

$$
F \llbracket X, Y \rrbracket=\llbracket F X, F Y \rrbracket^{\prime}, \quad \rho^{\prime}(F X)=\rho(X),
$$

for $X, Y \in \Gamma(E)$.

Let $(E, \llbracket \cdot, \cdot \rrbracket, \rho)$ be a Lie algebroid over a manifold $Q$ and $E^{*}$ be the dual bundle to $E$. Then, $E^{*}$ admits a linear Poisson structure $\{\cdot, \cdot\}_{E^{*}}$, that is,

$$
\{\cdot, \cdot\}_{E^{*}}: C^{\infty}\left(E^{*}\right) \times C^{\infty}\left(E^{*}\right) \rightarrow C^{\infty}\left(E^{*}\right)
$$

is a $\mathbb{R}$-bilinear map,

$$
\begin{array}{ll}
\{F, G\}_{E^{*}}=-\{G, F\}_{E^{*}}, & \text { (skew-symmetry), } \\
\left\{F F^{\prime}, G\right\}_{E^{*}}=F\left\{F^{\prime}, G\right\}_{E^{*}}+F^{\prime}\{F, G\}_{E^{*}}, & \text { (the Leibniz rule), } \\
\left\{F,\{G, H\}_{E^{*}}\right\}_{E^{*}}+\left\{G,\{H, F\}_{E^{*}}\right\}_{E^{*}} & \\
+\left\{H,\{F, G\}_{E^{*}}\right\}_{E^{*}}=0, & \text { (the Jacobi identity), }
\end{array}
$$

for $F, F^{\prime}, G, H \in C^{\infty}\left(E^{*}\right)$ and, in addition,

$$
P, P^{\prime} \text { linear functions on } E^{*} \Rightarrow\left\{P, P^{\prime}\right\}_{E^{*}} \text { is a linear function on } E^{*} \text {. }
$$

If $\left(x^{i}\right)$ are local coordinates on an open subset $U$ of $Q,\left\{e_{A}\right\}$ is a local basis of $\Gamma(E)$ on $U$ and $F, G \in C^{\infty}\left(E^{*}\right)$ then the local expression of the Poisson bracket of $F$ and $G$ is

$$
\{F, G\}_{E^{*}}=\rho_{A}^{i}\left(\frac{\partial F}{\partial x^{i}} \frac{\partial G}{\partial p_{A}}-\frac{\partial F}{\partial p_{A}} \frac{\partial G}{\partial x^{i}}\right)-\mathcal{C}_{A B}^{C} p_{C} \frac{\partial F}{\partial p_{A}} \frac{\partial G}{\partial p_{B}},
$$

where $\left(x^{i}, p_{A}\right)$ are the corresponding coordinates on $E^{*}$ (for more details, see [23]). 
2.1. The prolongation of a Lie algebroid over a fibration. Let $(E, \llbracket \cdot, \cdot \rrbracket, \rho)$ be a Lie algebroid of rank $n$ over a manifold $Q$ of dimension $m$ and $\pi: P \rightarrow Q$ be a fibration, that is, a surjective submersion.

We consider the subset $\mathcal{T}^{E} P$ of $E \times T P$ defined by $\mathcal{T}^{E} P=\bigcup_{p \in P} \mathcal{T}_{p}^{E} P$, where

$$
\mathcal{T}_{p}^{E} P=\left\{(b, v) \in E_{\pi(p)} \times T_{p} P \mid \rho(b)=\left(T_{p} \pi\right)(v)\right\}
$$

and $T \pi: T P \rightarrow T Q$ is the tangent map to $\pi$.

Denote by $\tau^{\pi}: \mathcal{T}^{E} P \rightarrow P$ the map given by

$$
\tau^{\pi}(b, v)=\tau_{P}(v)
$$

for $(b, v) \in \mathcal{T}^{E} P, \tau_{P}: T P \rightarrow P$ being the canonical projection. Then, if $m^{\prime}$ is the dimension of $P$, one may prove that

$$
\operatorname{dim} \mathcal{T}_{p}^{E} P=n+m^{\prime}-m
$$

Thus, we conclude that $\mathcal{T}^{E} P$ is a vector bundle over $P$ of rank $n+m^{\prime}-m$ with vector bundle projection $\tau^{\pi}: \mathcal{T}^{E} P \rightarrow P$.

A section $\tilde{X}$ of $\tau^{\pi}: \mathcal{T}^{E} P \rightarrow P$ is said to be projectable if there exists a section $X$ of $\tau: E \rightarrow Q$ and a vector field $U$ on $P$ which is $\pi$-projectable to the vector field $\rho(X)$ and such that $\tilde{X}(p)=(X(\pi(p)), U(p))$, for all $p \in P$. For such a projectable section $\tilde{X}$, we will use the following notation $\tilde{X} \equiv(X, U)$. It is easy to prove that one may choose a local basis of projectable sections of the space $\Gamma\left(\mathcal{T}^{E} P\right)$.

The vector bundle $\tau^{\pi}: \mathcal{T}^{E} P \rightarrow P$ admits a Lie algebroid structure $\left(\llbracket \cdot, \cdot \rrbracket^{\pi}, \rho^{\pi}\right)$. In fact,

$$
\llbracket\left(X_{1}, U_{1}\right),\left(X_{2}, U_{2}\right) \rrbracket^{\pi}=\left(\llbracket X_{1}, X_{2} \rrbracket,\left[U_{1}, U_{2}\right]\right), \quad \rho^{\pi}\left(X_{1}, U_{1}\right)=U_{1} .
$$

The Lie algebroid $\left(\mathcal{T}^{E} P, \llbracket \cdot, \cdot \rrbracket^{\pi}, \rho^{\pi}\right)$ is called the prolongation of $E$ over $\pi$ or the E-tangent bundle to $P$. Note that if $p r_{1}: \mathcal{T}^{E} P \rightarrow E$ is the canonical projection on the first factor, then the pair $\left(p r_{1}, \pi\right)$ is a morphism between the Lie algebroids $\left(\mathcal{T}^{E} P, \llbracket \cdot, \cdot \rrbracket^{\pi}, \rho^{\pi}\right)$ and $(E, \llbracket \cdot, \cdot \rrbracket, \rho)$ (for more details, see $[23]$ ).

Example 2.1. Let $(E, \llbracket \cdot, \cdot \rrbracket, \rho)$ be a Lie algebroid of rank $n$ over a manifold $Q$ of dimension $m$ and $\tau: E \rightarrow Q$ be the vector bundle projection. Consider the prolongation $\mathcal{T}^{E} E$ of $E$ over $\tau$,

$$
\mathcal{T}^{E} E=\{(e, v) \in E \times T E \mid \rho(e)=(T \tau)(v)\} .
$$

$\mathcal{T}^{E} E$ is a Lie algebroid over $E$ of rank $2 n$ with Lie algebroid structure $\left(\llbracket \cdot, \cdot \rrbracket^{\tau}, \rho^{\tau}\right)$.

If $\left(x^{i}\right)$ are local coordinates on an open subset $U$ of $Q$ and $\left\{e_{A}\right\}$ is a basis of sections of the vector bundle $\tau^{-1}(U) \rightarrow U$, then $\left\{X_{A}, \mathcal{V}_{A}\right\}$ is a basis of sections of the vector bundle $\left(\tau^{\tau}\right)^{-1}\left(\tau^{-1}(U)\right) \rightarrow \tau^{-1}(U)$, where $\tau^{\tau}: \mathcal{T}^{E} E \rightarrow E$ is the vector bundle projection and

$$
\begin{aligned}
& x_{A}(e)=\left(e_{A}(\tau(e)), \rho_{A}^{i} \frac{\partial}{\partial x^{i} \mid e}\right), \\
& \nu_{A}(e)=\left(0, \frac{\partial}{\partial y^{A}}\right),
\end{aligned}
$$

for $e \in \tau^{-1}(U)$. Here, $\rho_{A}^{i}$ are the components of the anchor map with respect to the basis $\left\{e_{A}\right\}$ and $\left(x^{i}, y^{A}\right)$ are the local coordinates on $E$ induced by the local coordinates $\left(x^{i}\right)$ and the basis $\left\{e_{A}\right\}$. Using the local basis $\left\{X_{A}, V_{A}\right\}$, one may introduce, in a natural way, local coordinates $\left(x^{i}, y^{A} ; z^{A}, v^{A}\right)$ on $\mathcal{T}^{E} E$. On the 
other hand, we have that

$$
\begin{aligned}
\rho^{\tau}\left(X_{A}\right) & =\rho_{A}^{i} \frac{\partial}{\partial x^{i}}, \quad \rho^{\tau}\left(\mathcal{V}_{A}\right)=\frac{\partial}{\partial y^{A}}, \\
\llbracket X_{A}, X_{B} \rrbracket^{\tau} & =e_{A B}^{C} X_{C}, \\
\llbracket X_{A}, \mathcal{V}_{B} \rrbracket^{\tau} & =\llbracket \mathcal{V}_{A}, \mathcal{V}_{B} \rrbracket^{\tau}=0,
\end{aligned}
$$

for all $A$ and $B, \mathcal{C}_{A B}^{C}$ being the structure functions of the Lie bracket $\llbracket \cdot, \cdot \rrbracket$ with respect to the basis $\left\{e_{A}\right\}$.

The vector subbundle $\left(\mathcal{T}^{E} E\right)^{V}$ of $\mathcal{T}^{E} E$ whose fibre at the point $e \in E$ is

$$
\left(\mathcal{T}_{e}^{E} E\right)^{V}=\left\{(0, v) \in E \times T_{e} E /\left(T_{e} \tau\right)(v)=0\right\}
$$

is called the vertical subbundle. Note that $\left(\mathcal{T}^{E} E\right)^{V}$ is locally generated by the sections $\left\{\mathcal{V}_{A}\right\}$.

Two canonical objects on $\mathcal{T}^{E} E$ are the Euler section $\Delta$ and the vertical endomorphism $S . \Delta$ is the section of $\mathcal{T}^{E} E \rightarrow E$ locally defined by

$$
\Delta=y^{A} \mathcal{V}_{A},
$$

and $S$ is the section of the vector bundle $\left(\mathcal{T}^{E} E\right) \otimes\left(\mathcal{T}^{E} E\right)^{*} \rightarrow E$ locally characterized by the following conditions

$$
S X_{A}=\mathcal{V}_{A}, \quad S \mathcal{V}_{A}=0, \quad \text { for all } A .
$$

Finally, a section $\xi$ of $\mathcal{T}^{E} E \rightarrow E$ is said to be a second order differential equation (SODE) on $E$ if $S(\xi)=\Delta$ or, alternatively, $p r_{1}(\xi(e))=e$, for all $e \in E$ (for more details, see [23]).

Example 2.2. Let $(E, \llbracket \cdot, \cdot \rrbracket, \rho)$ be a Lie algebroid of rank $n$ over a manifold $Q$ of dimension $m$ and $\tau^{*}: E^{*} \rightarrow Q$ be the vector bundle projection of the dual bundle $E^{*}$ to $E$.

We consider the prolongation $\mathcal{T}^{E} E^{*}$ of $E$ over $\tau^{*}$,

$$
\mathcal{T}^{E} E^{*}=\left\{\left(e^{\prime}, v\right) \in E \times T E^{*} \mid \rho\left(e^{\prime}\right)=\left(T \tau^{*}\right)(v)\right\} .
$$

$\mathcal{T}^{E} E^{*}$ is a Lie algebroid over $E^{*}$ of rank $2 n$ with Lie algebroid structure $\left(\llbracket \cdot, \cdot \rrbracket^{\tau^{*}}, \rho^{\tau^{*}}\right)$.

If $\left(x^{i}\right)$ are local coordinates on an open subset $U$ of $Q,\left\{e_{A}\right\}$ is a basis of sections of the vector bundle $\tau^{-1}(U) \rightarrow U$ and $\left\{e^{A}\right\}$ is the dual basis of $\left\{e_{A}\right\}$, then $\left\{y_{A}, \mathcal{P}^{A}\right\}$ is a basis of sections of the vector bundle $\left(\tau^{\tau^{*}}\right)^{-1}\left(\left(\tau^{*}\right)^{-1}(U)\right) \rightarrow\left(\tau^{*}\right)^{-1}(U)$, where $\tau^{\tau^{*}}: \mathcal{T}^{E} E^{*} \rightarrow E^{*}$ is the vector bundle projection and

$$
\begin{aligned}
& y_{A}\left(e^{*}\right)=\left(e_{A}\left(\tau^{*}\left(e^{*}\right)\right), \rho_{A}^{i} \frac{\partial}{\partial x^{i} \mid e^{*}}\right), \\
& \mathcal{P}^{A}\left(e^{*}\right)=\left(0,\left.\frac{\partial}{\partial p_{A}}\right|_{e^{*}}\right),
\end{aligned}
$$

for $e^{*} \in\left(\tau^{*}\right)^{-1}(U)$. Here, $\left(x^{i}, p_{A}\right)$ are the local coordinates on $E^{*}$ induced by the local coordinates $\left(x^{i}\right)$ and the basis $\left\{e^{A}\right\}$ of $\Gamma\left(E^{*}\right)$. Using the local basis $\left\{y_{A}, \mathcal{P}^{A}\right\}$, one may introduce, in a natural way, local coordinates $\left(x^{i}, p_{A} ; u^{A}, q_{A}\right)$ on $\mathcal{T}^{E} E^{*}$. If $\omega^{*}$ is a point of $\left(\tau^{\tau^{*}}\right)^{-1}\left(\left(\tau^{*}\right)^{-1}(U)\right)$, then $\left(x^{i}, p_{A}\right)$ are the coordinates of the point $\tau^{\tau^{*}}\left(\omega^{*}\right) \in\left(\tau^{*}\right)^{-1}(U)$ and

$$
\omega^{*}=u^{A} y_{A}\left(\tau^{\tau^{*}}\left(\omega^{*}\right)\right)+q_{A} \mathcal{P}^{A}\left(\tau^{\tau^{*}}\left(\omega^{*}\right)\right) .
$$


On the other hand, we have that

$$
\begin{aligned}
\rho^{\tau^{*}}\left(y_{A}\right) & =\rho_{A}^{i} \frac{\partial}{\partial x^{i}}, \quad \rho^{\tau^{*}}\left(\mathcal{P}^{A}\right)=\frac{\partial}{\partial p_{A}}, \\
\llbracket y_{A}, y_{B} \rrbracket^{\tau^{*}} & =\mathcal{C}_{A B}^{C} y_{C}, \\
\llbracket y_{A}, \mathcal{P}^{B} \rrbracket \tau^{*} & =\llbracket \mathcal{P}^{A}, \mathcal{P}^{B} \rrbracket^{\tau^{*}}=0,
\end{aligned}
$$

for all $A$ and $B$. Thus, if $\left\{y^{A}, \mathcal{P}_{A}\right\}$ is the dual basis of $\left\{y_{A}, \mathcal{P} A\right\}$, then

$$
\begin{aligned}
d^{\mathfrak{T}^{E}} E^{*} f & =\rho_{A}^{i} \frac{\partial f}{\partial x^{i}} y^{A}+\frac{\partial f}{\partial p_{A}} \mathcal{P}_{A}, \\
d^{\mathfrak{T}^{E}} E^{*} y C & =-\frac{1}{2} \bigodot_{A B}^{C} y^{A} \wedge y^{B}, \quad d^{\mathfrak{T}^{E} E^{*}} \mathcal{P}_{C}=0,
\end{aligned}
$$

for $f \in C^{\infty}\left(E^{*}\right)$.

We may introduce a canonical section $\lambda_{E}$ of the vector bundle $\left(\mathcal{T}^{E} E^{*}\right)^{*} \rightarrow E^{*}$ as follows. If $e^{*} \in E^{*}$ and $(\tilde{e}, v)$ is a point of the fiber of $\mathcal{T}^{E} E^{*}$ over $e^{*}$, then

$$
\lambda_{E}\left(e^{*}\right)(\tilde{e}, v)=e^{*}(\tilde{e}) .
$$

$\lambda_{E}$ is called the Liouville section of $\left(\mathcal{T}^{E} E^{*}\right)^{*}$.

Now, the canonical symplectic section $\Omega_{E}$ is the nondegenerate closed 2section defined by

$$
\Omega_{E}=-d^{\mathcal{T}^{E}} E^{*} \lambda_{E}
$$

In local coordinates,

$$
\begin{gathered}
\lambda_{E}\left(x^{i}, p_{A}\right)=p_{A} y^{A}, \\
\Omega_{E}=y^{A} \wedge \mathcal{P}_{A}+\frac{1}{2} \mathcal{C}_{A B}^{C} p_{C} y^{A} \wedge y^{B},
\end{gathered}
$$

(for more details, see [23]).

2.2. Lagrangian and Hamiltonian mechanics on Lie algebroids. Given a Lagrangian function $L \in C^{\infty}(E)$ we define the Cartan 1-section $\theta_{L} \in \Gamma\left(\left(\mathcal{T}^{E} E\right)^{*}\right)$, the Cartan 2-section $\omega_{L} \in \Gamma\left(\wedge^{2}\left(\mathcal{T}^{E} E\right)^{*}\right)$ and the Lagrangian energy $E_{L} \in$ $C^{\infty}(E)$ as

$$
\theta_{L}=S^{*}\left(d^{\mathcal{T}^{E} E} L\right), \quad \omega_{L}=-d^{\mathcal{T}^{E} E} \theta_{L} \quad \text { and } \quad E_{L}=\mathcal{L}_{\Delta}^{\mathcal{T}^{E} E} L-L .
$$

If $\left(x^{i}, y^{A}\right)$ are local fibred coordinates on $E,\left(\rho_{A}^{i}, \mathcal{C}_{A B}^{C}\right)$ are the corresponding local structure functions on $E$ and $\left\{X_{A}, \mathcal{V}_{A}\right\}$ is the corresponding local basis of sections of $\mathcal{T}^{E} E$ then

$$
\begin{gathered}
\omega_{L}=\frac{\partial^{2} L}{\partial y^{A} \partial y^{B}} X^{A} \wedge \mathcal{V}^{B}+\frac{1}{2}\left(\frac{\partial^{2} L}{\partial x^{i} \partial y^{A}} \rho_{B}^{i}-\frac{\partial^{2} L}{\partial x^{i} \partial y^{B}} \rho_{A}^{i}+\frac{\partial L}{\partial y^{C}} \varrho_{A B}^{C}\right) X^{A} \wedge X^{B} \\
E_{L}=\frac{\partial L}{\partial y^{A}} y^{A}-L .
\end{gathered}
$$

From (2.3), (2.4), (2.6) and (2.7), it follows that

$$
i_{S X} \omega_{L}=-S^{*}\left(i_{X} \omega_{L}\right), \quad i_{\Delta} \omega_{L}=-S^{*}\left(d^{\mathcal{T}^{E}} E E_{L}\right),
$$

for $X \in \Gamma\left(\mathcal{T}^{E} E\right)$.

Now, a curve $t \rightarrow c(t)$ on $E$ is a solution of the Euler-Lagrange equations for $L$ if

- $c$ is admissible (that is, $\rho(c(t))=\dot{m}(t)$, where $m=\tau \circ c$ ) and

- $i_{(c(t), \dot{c}(t))} \omega_{L}(c(t))-d^{\mathcal{T}^{E}} E^{E} E_{L}(c(t))=0$, for all $t$. 
If $c(t)=\left(x^{i}(t), y^{A}(t)\right)$ then $c$ is a solution of the Euler-Lagrange equations for $L$ if and only if

$$
\dot{x}^{i}=\rho_{A}^{i} y^{A}, \quad \frac{d}{d t}\left(\frac{\partial L}{\partial y^{A}}\right)+\frac{\partial L}{\partial y^{C}} \varrho_{A B}^{C} y^{B}-\rho_{A}^{i} \frac{\partial L}{\partial x^{i}}=0 .
$$

Note that if $E$ is the standard Lie algebroid $T Q$ then the above equations are the classical Euler-Lagrange equations for $L: T Q \rightarrow \mathbb{R}$.

On the other hand, the Lagrangian function $L$ is said to be regular if $\omega_{L}$ is a symplectic section, that is, if $\omega_{L}$ is regular at every point as a bilinear form. In such a case, there exists a unique solution $\xi_{L}$ verifying

$$
i_{\xi_{L}} \omega_{L}-d^{\mathcal{T}^{E}} E_{L}=0
$$

In addition, using (2.8), it follows that $i_{S \xi_{L}} \omega_{L}=i_{\Delta} \omega_{L}$ which implies that $\xi_{L}$ is a SODE section. Thus, the integral curves of $\xi_{L}$ (that is, the integral curves of the vector field $\rho^{\tau}\left(\xi_{L}\right)$ ) are solutions of the Euler-Lagrange equations for $L$. $\xi_{L}$ is called the Euler-Lagrange section associated with $L$.

¿From (2.6), we deduce that the Lagrangian $L$ is regular if and only if the matrix $\left(W_{A B}\right)=\left(\frac{\partial^{2} L}{\partial y^{A} \partial y^{B}}\right)$ is regular. Moreover, the local expression of $\xi_{L}$ is

$$
\xi_{L}=y^{A} x_{A}+f^{A} \nu_{A},
$$

where the functions $f^{A}$ satisfy the linear equations

$$
\frac{\partial^{2} L}{\partial y^{B} \partial y^{A}} f^{B}+\frac{\partial^{2} L}{\partial x^{i} \partial y^{A}} \rho_{B}^{i} y^{B}+\frac{\partial L}{\partial y^{C}} e_{A B}^{C} y^{B}-\rho_{A}^{i} \frac{\partial L}{\partial x^{i}}=0, \text { for all } A
$$

Another possibility is when the matrix $\left(W_{A B}\right)=\left(\frac{\partial^{2} L}{\partial y^{A} \partial y^{B}}\right)$ is non regular. This type of lagrangians are called singular or degenerate lagrangians. In such a case, $\omega_{L}$ is not symplectic and Equation (2.10) has no solution, in general, and even if it exists it will not be unique. In the next section, we will extend the classical Gotay-Nester-Hinds algorithm [13] for presymplectic systems on Lie algebroids, which in particular will be applied to the case of singular lagrangians in Section 4 ,

For an arbitrary Lagrangian function $L: E \rightarrow \mathbb{R}$, we introduce the Legendre transformation associated with $L$ as the smooth map $\operatorname{leg}_{L}: E \rightarrow E^{*}$ defined by

$$
\operatorname{leg}_{L}(e)\left(e^{\prime}\right)=\theta_{L}(e)\left(z^{\prime}\right),
$$

for $e, e^{\prime} \in E_{x}$, where $z^{\prime} \in \mathcal{T}_{e}^{E} E \subseteq E_{x} \times T_{e} E$ satisfies

$$
\operatorname{pr}_{1}\left(z^{\prime}\right)=e^{\prime},
$$

$p r_{1}: \mathcal{T}^{E} E \rightarrow E$ being the restriction to $\mathcal{T}^{E} E$ of the canonical projection $p r_{1}$ : $E \times T E \rightarrow E$. The map $\operatorname{leg}_{L}: E \rightarrow E^{*}$ is well defined and its local expression is

$$
\operatorname{leg}_{L}\left(x^{i}, y^{A}\right)=\left(x^{i}, \frac{\partial L}{\partial y^{A}}\right)
$$

The Legendre transformation induces a Lie algebroid morphism

$$
\mathcal{T} \operatorname{leg}_{L}: \mathcal{T}^{E} E \rightarrow \mathcal{T}^{E} E^{*}
$$

over $\operatorname{leg}_{L}: E \rightarrow E^{*}$ given by

$$
\left(\mathcal{T} \operatorname{leg}_{L}\right)(e, v)=\left(e,\left(\operatorname{Tleg}_{L}\right)(v)\right),
$$

where $\operatorname{Tleg}_{L}: T E \rightarrow T E^{*}$ is the tangent map to $\operatorname{leg}_{L}: E \rightarrow E^{*}$.

We have that (see [23])

$$
\left(\mathcal{T} \operatorname{leg}_{L}, \operatorname{leg}_{L}\right)^{*}\left(\lambda_{E}\right)=\Theta_{L}, \quad\left(\mathcal{T} \operatorname{leg}_{L}, \operatorname{leg}_{L}\right)^{*}\left(\Omega_{E}\right)=\omega_{L} .
$$


On the other hand, from (2.11), it follows that the Lagrangian function $L$ is regular if and only if $\operatorname{leg}_{L}: E \rightarrow E^{*}$ is a local diffeomorphism.

Next, we will assume that $L$ is hyperregular, that is, $\operatorname{leg}_{L}: E \rightarrow E^{*}$ is a global diffeomorphism. Then, the pair $\left(\mathcal{T} l e g_{L}, \operatorname{leg}_{L}\right)$ is a Lie algebroid isomorphism. Moreover, we may consider the Hamiltonian function $H: E^{*} \rightarrow \mathbb{R}$ defined by

$$
H=E_{L} \circ \operatorname{leg}_{L}^{-1}
$$

and the Hamiltonian section $\xi_{H} \in \Gamma\left(\mathcal{T}^{E} E^{*}\right)$ which is characterized by the condition

$$
i_{\xi_{H}} \Omega_{E}=d^{\mathcal{T}^{E} E^{*}} H
$$

The integral curves of the vector field $\rho^{\tau^{*}}\left(\xi_{H}\right)$ on $E^{*}$ satisfy the Hamilton equations for $H$

$$
\frac{d x^{i}}{d t}=\rho_{A}^{i} \frac{\partial H}{\partial p_{A}}, \quad \frac{d p_{A}}{d t}=-\left(\rho_{A}^{i} \frac{\partial H}{\partial x^{i}}+\bigodot_{A B}^{C} p_{C} \frac{\partial H}{\partial p_{B}}\right)
$$

for $i \in\{1, \ldots, m\}$ and $A \in\{1, \ldots, n\}$ (see [23]).

In addition, the Euler-Lagrange section $\xi_{L}$ associated with $L$ and the Hamiltonian section $\xi_{H}$ are $\left(\mathcal{T} \operatorname{leg}_{L}, \operatorname{leg}_{L}\right)$-related, that is,

$$
\xi_{H} \circ \operatorname{leg}_{L}=\mathcal{T} \operatorname{leg}_{L} \circ \xi_{L} .
$$

Thus, if $\gamma: I \rightarrow E$ is a solution of the Euler-Lagrange equations associated with $L$, then $\mu=\operatorname{leg}_{L} \circ \gamma: I \rightarrow E^{*}$ is a solution of the Hamilton equations for $H$ and, conversely, if $\mu: I \rightarrow E^{*}$ is a solution of the Hamilton equations for $H$ then $\gamma=\operatorname{leg}_{L}^{-1} \circ \mu$ is a solution of the Euler-Lagrange equations for $L$ (for more details, see [23]).

\section{Constraint Algorithm And Reduction for PResymplectic Lie ALGEBROIDS}

3.1. Constraint algorithm for presymplectic Lie algebroids. Let $\tau: E \rightarrow Q$ be a Lie algebroid and suppose that $\Omega \in \Gamma\left(\wedge^{2} E^{*}\right)$. Then, we can define the vector bundle morphism $b_{\Omega}: E \rightarrow E^{*}$ (over the identity of $Q$ ) as follows

$$
b_{\Omega}(e)=i(e) \Omega(x), \text { for } e \in E_{x} .
$$

Now, if $x \in Q$ and $F_{x}$ is a subspace of $E_{x}$, we may introduce the vector subspace $F_{x}^{\perp}$ of $E_{x}$ given by

$$
F_{x}^{\perp}=\left\{e \in E_{x} \mid \Omega(x)(e, f)=0, \forall f \in F_{x}\right\} .
$$

Then, using a well-known result (see, for instance, [24]), we have that

$$
\operatorname{dim} F_{x}^{\perp}=\operatorname{dim} E_{x}-\operatorname{dim} F_{x}+\operatorname{dim}\left(E_{x}^{\perp} \cap F_{x}\right) .
$$

On the other hand, if $b_{\Omega_{x}}=b_{\Omega \mid E_{x}}$ it is easy to prove that

$$
b_{\Omega_{x}}\left(F_{x}\right) \subseteq\left(F_{x}^{\perp}\right)^{0},
$$

where $\left(F_{x}^{\perp}\right)^{0}$ is the annihilator of the subspace $F_{x}^{\perp}$. Moreover, using (3.1), we obtain that

$$
\operatorname{dim}\left(F_{x}^{\perp}\right)^{0}=\operatorname{dim} F_{x}-\operatorname{dim}\left(E_{x}^{\perp} \cap F_{x}\right)=\operatorname{dim}\left(b_{\Omega_{x}}\left(F_{x}\right)\right) .
$$

Thus, from (3.2), we deduce that

$$
b_{\Omega_{x}}\left(F_{x}\right)=\left(F_{x}^{\perp}\right)^{\circ} .
$$


Next, we will assume that $\Omega$ is a presymplectic 2 -section $\left(d^{E} \Omega=0\right)$ and that $\alpha \in \Gamma\left(E^{*}\right)$ is a closed 1-section $\left(d^{E} \alpha=0\right)$. Furthermore, we will assume that the kernel of $\Omega$ is a vector subbundle of $E$.

The dynamics of the presymplectic system defined by $(\Omega, \alpha)$ is given by a section $X \in \Gamma(E)$ satisfying the dynamical equation

$$
i_{X} \Omega=\alpha \text {. }
$$

In general, a section $X$ satisfying (3.4) cannot be found in all points of $E$. First, we look for the points where (3.4) has sense. We define

$$
Q_{1}=\left\{x \in Q \mid \exists e \in E_{x}: i(e) \Omega(x)=\alpha(x)\right\}=\left\{x \in Q \mid \alpha(x) \in b_{\Omega_{x}}\left(E_{x}\right)\right\} .
$$

¿From (3.3), it follows that

$$
Q_{1}=\left\{x \in Q \mid \alpha(x)(e)=0, \text { for all } e \in \operatorname{Ker} \Omega(x)=E_{x}^{\perp}\right\} .
$$

If $Q_{1}$ is an embedded submanifold of $Q$, then we deduce that there exists $X$ : $Q_{1} \rightarrow E$ a section of $\tau: E \rightarrow Q$ along $Q_{1}$ such that (3.4) holds. But $\rho(X)$ is not, in general, tangent to $Q_{1}$. Thus, we have that to restrict to $E_{1}=\rho^{-1}\left(T Q_{1}\right)$. We remark that, provided that $E_{1}$ is a manifold and $\tau_{1}=\tau_{\mid E_{1}}: E_{1} \rightarrow Q_{1}$ is a vector bundle, $\tau_{1}: E_{1} \rightarrow Q_{1}$ is a Lie subalgebroid of $E \rightarrow Q$.

Now, we must consider the subset $Q_{2}$ of $Q_{1}$ defined by

$$
\begin{aligned}
Q_{2} & =\left\{x \in Q_{1} \mid \alpha(x) \in b_{\Omega_{x}}\left(\left(E_{1}\right)_{x}\right)=b_{\Omega_{x}}\left(\rho^{-1}\left(T_{x} Q_{1}\right)\right)\right\} \\
& =\left\{x \in Q_{1} \mid \alpha(x)(e)=0, \text { for all } e \in\left(E_{1}\right)_{x}^{\perp}=\left(\rho^{-1}\left(T_{x} Q_{1}\right)\right)^{\perp}\right\} .
\end{aligned}
$$

If $Q_{2}$ is an embedded submanifold of $Q_{1}$, then we deduce that there exists $X$ : $Q_{2} \rightarrow E_{1}$ a section of $\tau_{1}: E_{1} \rightarrow Q_{1}$ along $Q_{2}$ such that (3.4) holds. However, $\rho(X)$ is not, in general, tangent to $Q_{2}$. Therefore, we have that to restrict to $E_{2}=\rho^{-1}\left(T Q_{2}\right)$. As above, if $\tau_{2}=\tau_{\mid E_{2}}: E_{2} \rightarrow Q_{2}$ is a vector bundle, it follows that $\tau_{2}: E_{2} \rightarrow Q_{2}$ is a Lie subalgebroid of $\tau_{1}: E_{1} \rightarrow Q_{1}$.

Consequently, if we repeat the process, we obtain a sequence of Lie subalgebroids (by assumption):

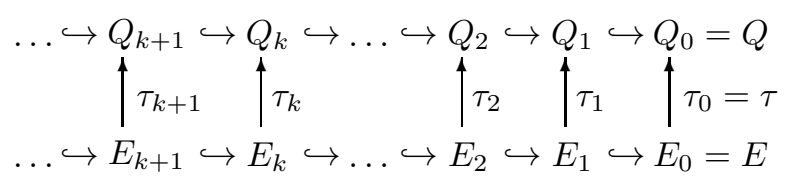

where

$$
Q_{k+1}=\left\{x \in Q_{k} \mid \alpha(x)(e)=0, \text { for all } e \in\left(\rho^{-1}\left(T_{x} Q_{k}\right)\right)^{\perp}\right\}
$$

and

$$
E_{k+1}=\rho^{-1}\left(T Q_{k+1}\right) .
$$

If there exists $k \in \mathbb{N}$ such that $Q_{k}=Q_{k+1}$, then we say that the sequence stabilizes. In such a case, there exists a well-defined (but non necessarily unique) dynamics on the final constraint submanifold $Q_{f}=Q_{k}$. We write

$$
Q_{f}=Q_{k+1}=Q_{k}, \quad E_{f}=E_{k+1}=E_{k}=\rho^{-1}\left(T Q_{k}\right) .
$$

Then, $\tau_{f}=\tau_{k}: E_{f}=E_{k} \rightarrow Q_{f}=Q_{k}$ is a Lie subalgebroid of $\tau: E \longrightarrow Q$ (the Lie algebroid restriction of $E$ to $E_{f}$ ). From the construction of the constraint algorithm, we deduce that there exists a section $X \in \Gamma\left(E_{f}\right)$, verifying (3.4). Moreover, if $X \in \Gamma\left(E_{f}\right)$ is a solution of the equation (3.4), then every arbitrary solution is of the form $X^{\prime}=X+Y$, where $Y \in \Gamma\left(E_{f}\right)$ and $Y(x) \in \operatorname{ker} \Omega(x)$, for all $x \in Q_{f}$. In addition, if we denote by $\Omega_{f}$ and $\alpha_{f}$ the restriction of $\Omega$ and $\alpha$, respectively, to the 
Lie algebroid $E_{f} \longrightarrow Q_{f}$, we have that $\Omega_{f}$ is a presymplectic 2-section and then any $X \in \Gamma\left(E_{f}\right)$ verifying Equation (3.4) also satisfies

$$
i_{X} \Omega_{f}=\alpha_{f}
$$

but, in principle, there are solutions of (3.7) which are not solutions of (3.4) since $\operatorname{ker} \Omega \cap E_{f} \subset \operatorname{ker} \Omega_{f}$.

Remark 3.1. Note that one can generalize the previous procedure to the general setting of implicit differential equations on a Lie algebroid. More precisely, let $\tau: E \rightarrow Q$ be a Lie algebroid and $S \subset E$ be a submanifold of $E$ (not necessarily a vector subbundle). Then, the corresponding sequence of submanifolds of $E$ is

$$
\begin{array}{cl}
S_{0} & =S \\
S_{1} & =S_{0} \cap \rho^{-1}\left(T \tau\left(S_{0}\right)\right) \\
\vdots & \\
S_{k+1} & =S_{k} \cap \rho^{-1}\left(T \tau\left(S_{k}\right)\right)
\end{array}
$$

In our case, $S_{k}=\rho^{-1}\left(T Q_{k}\right)$ (equivalently, $Q_{k}=\tau\left(S_{k}\right)$ ).

3.2. Reduction of presymplectic Lie algebroids. Let $(E, \llbracket \cdot, \cdot \rrbracket, \rho)$ and $\left(E^{\prime}, \llbracket \cdot, \cdot \rrbracket^{\prime}\right.$, $\left.\rho^{\prime}\right)$ be two Lie algebroids over $Q$ and $Q^{\prime}$, respectively. Suppose that $\Omega \in \Gamma\left(\wedge^{2} E^{*}\right)$ (respectively, $\Omega^{\prime} \in \Gamma\left(\wedge^{2}\left(E^{\prime}\right)^{*}\right)$ ) is a presymplectic 2-section on $\tau: E \rightarrow Q$ (respectively, $\tau^{\prime}: E^{\prime} \rightarrow Q^{\prime}$ ) and that $\alpha \in \Gamma\left(E^{*}\right)$ (respectively, $\alpha^{\prime} \in \Gamma\left(\left(E^{\prime}\right)^{*}\right)$ ) is a closed 1-section on $\tau: E \rightarrow Q$ (respectively, $\tau^{\prime}: E^{\prime} \rightarrow Q^{\prime}$ ). Then, we may consider the corresponding dynamical equations

$$
\begin{gathered}
i_{X} \Omega=\alpha, \quad X \in \Gamma(E), \\
i_{X^{\prime}} \Omega^{\prime}=\alpha^{\prime}, \quad X^{\prime} \in \Gamma\left(E^{\prime}\right) .
\end{gathered}
$$

If we apply our constraint algorithm to the first problem, we will obtain a sequence of Lie subalgebroids of $\tau: E \rightarrow Q$

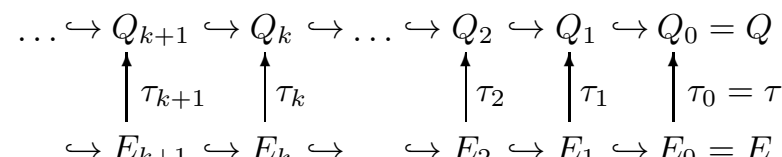

In a similar way, if we apply our constraint algorithm to the second problem, we will obtain a sequence of Lie subalgebroids of $\tau^{\prime}: E^{\prime} \rightarrow Q^{\prime}$

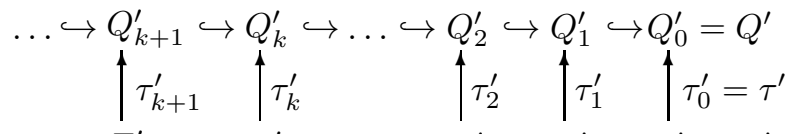

$$
\begin{aligned}
& \ldots \hookrightarrow E_{k+1}^{\prime} \hookrightarrow E_{k}^{\prime} \hookrightarrow \ldots \hookrightarrow E_{2}^{\prime} \hookrightarrow E_{1}^{\prime} \hookrightarrow E_{0}^{\prime}=E^{\prime}
\end{aligned}
$$

On the other hand, it is clear that the restriction $\Omega_{k}$ (respectively, $\Omega_{k}^{\prime}$ ) of $\Omega$ (respectively, $\Omega^{\prime}$ ) to the Lie subalgebroid $\tau_{k}: E_{k} \rightarrow Q_{k}$ (respectively, $\tau_{k}^{\prime}: E_{k}^{\prime} \rightarrow Q_{k}^{\prime}$ ) is a presymplectic section of $\tau_{k}: E_{k} \rightarrow Q_{k}$ (respectively, $\tau_{k}^{\prime}: E_{k}^{\prime} \rightarrow Q_{k}^{\prime}$ ). Moreover, if $\alpha_{k}$ (respectively, $\alpha_{k}^{\prime}$ ) is the restriction of $\alpha$ (respectively, $\alpha^{\prime}$ ) to $\tau_{k}: E_{k} \rightarrow Q_{k}$ (respectively, $\tau_{k}^{\prime}: E_{k}^{\prime} \rightarrow Q_{k}^{\prime}$ ), we may consider the dynamical problem

$$
i_{X_{k}} \Omega_{k}=\alpha_{k}, \quad X_{k} \in \Gamma\left(E_{k}\right),
$$

(respectively, $i_{X_{k}^{\prime}} \Omega_{k}^{\prime}=\alpha_{k}^{\prime}, \quad X_{k}^{\prime} \in \Gamma\left(E_{k}^{\prime}\right)$ ) on $\tau_{k}: E_{k} \rightarrow Q_{k}$ (respectively, $\tau_{k}^{\prime}: E_{k}^{\prime} \rightarrow$ $\left.Q_{k}^{\prime}\right)$. 
Now, suppose that the pair $(\Pi, \pi)$

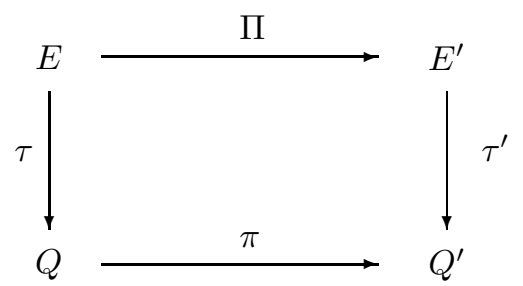

is a dynamical Lie algebroid epimorphism between $E$ and $E^{\prime}$. This means that:

(i) The pair $(\Pi, \pi)$ is a Lie algebroid morphism,

(ii) $\pi: Q \rightarrow Q^{\prime}$ is a surjective submersion and $\Pi_{\mid E_{x}}: E_{x} \rightarrow E_{\pi(x)}^{\prime}$ is a linear epimorphism, for all $x \in Q$, and

(iii) $(\Pi, \pi)^{*} \Omega^{\prime}=\Omega$ and $(\Pi, \pi)^{*} \alpha^{\prime}=\alpha$.

Then, we will see that the Lie subalgebroids in the two above sequences are related by dynamical Lie algebroid epimorphisms. First, we will prove the result for $k=1$.

Lemma 3.2. If $(\Pi, \pi)$ is a dynamical Lie algebroid epimorphism, we have that:

a) $\pi\left(Q_{1}\right)=Q_{1}^{\prime}$ and $\Pi\left(E_{1}\right)=E_{1}^{\prime}$.

b) If $x_{1} \in Q_{1}$, then $\pi^{-1}\left(\pi\left(x_{1}\right)\right) \subseteq Q_{1}$ and $\operatorname{Ker}\left(\Pi_{\mid E_{x_{1}}}\right) \subseteq\left(E_{1}\right)_{x_{1}}$.

c) If $\pi_{1}: Q_{1} \rightarrow Q_{1}^{\prime}$ and $\Pi_{1}: E_{1} \rightarrow E_{1}^{\prime}$ are the restrictions to $Q_{1}$ and $E_{1}$ of $\pi: Q \rightarrow Q^{\prime}$ and $\Pi: E \rightarrow E^{\prime}$, respectively, then the pair $\left(\Pi_{1}, \pi_{1}\right)$ is a dynamical Lie algebroid epimorphism.

Proof. If $x \in Q$ then, using that $(\Pi, \pi)^{*} \Omega^{\prime}=\Omega$ and the fact that $\Pi_{\mid E_{x}}: E_{x} \rightarrow E_{\pi(x)}^{\prime}$ is a linear epimorphism, we deduce that

$$
\Pi(\operatorname{Ker} \Omega(x))=\operatorname{Ker} \Omega^{\prime}(\pi(x)) .
$$

Thus, from (3.5), (3.8) and since $(\Pi, \pi)^{*} \alpha^{\prime}=\alpha$, it follows that

$$
\pi\left(Q_{1}\right) \subseteq Q_{1}^{\prime} .
$$

Conversely, if $x_{1}^{\prime} \in Q_{1}^{\prime}$ and $x \in \pi^{-1}\left(x_{1}^{\prime}\right)$ then, using again (3.5), (3.8) and the fact that $(\Pi, \pi)^{*} \alpha^{\prime}=\alpha$, we obtain that $x \in Q_{1}$. This proves that

$$
Q_{1}^{\prime} \subseteq \pi\left(Q_{1}\right)
$$

and the following result

$$
x_{1} \in Q_{1} \Rightarrow \pi^{-1}\left(\pi\left(x_{1}\right)\right) \subseteq Q_{1} .
$$

Now, we will see that $\pi_{1}: Q_{1} \rightarrow Q_{1}^{\prime}$ is a submersion.

In fact, if $x_{1} \in Q_{1}$ and $x_{1}^{\prime}=\pi_{1}\left(x_{1}\right)$ then there exist an open subset $U^{\prime}$ of $Q^{\prime}$, $x_{1}^{\prime} \in U^{\prime}$, and an smooth local section $s^{\prime}: U^{\prime} \rightarrow Q$ of the submersion $\pi: Q \rightarrow Q^{\prime}$ such that $s^{\prime}\left(x_{1}^{\prime}\right)=x_{1}$. Note that, using (3.9), we conclude that the restriction $s_{1}^{\prime}$ of $s^{\prime}$ to the open subset $U_{1}^{\prime}=U^{\prime} \cap Q_{1}^{\prime}$ of $Q_{1}^{\prime}$ takes values in $Q_{1}$. Therefore, $s_{1}^{\prime}: U_{1}^{\prime} \subseteq Q_{1}^{\prime} \rightarrow Q_{1}$ is a smooth map, $s_{1}^{\prime}\left(x_{1}^{\prime}\right)=x_{1}$ and $s_{1}^{\prime} \circ \pi_{1}=I d$. Consequently, $\pi_{1}: Q_{1} \rightarrow Q_{1}^{\prime}$ is a submersion.

Next, we will prove that

$$
\Pi\left(\left(E_{1}\right)_{x_{1}}\right)=\Pi\left(\rho^{-1}\left(T_{x_{1}} Q_{1}\right)\right)=\left(\rho^{\prime}\right)^{-1}\left(T_{\pi\left(x_{1}\right)} Q_{1}^{\prime}\right)=\left(E_{1}^{\prime}\right)_{\pi\left(x_{1}\right)} \text {, for } x_{1} \in Q_{1} .
$$


Since

it follows that

$$
\left(\rho^{\prime} \circ \Pi\right)_{\mid E_{x_{1}}}=(T \pi \circ \rho)_{\mid E_{x_{1}}},
$$

$$
\Pi\left(\left(E_{1}\right)_{x_{1}}\right) \subseteq\left(E_{1}^{\prime}\right)_{\pi\left(x_{1}\right)}
$$

Conversely, suppose that $e_{1}^{\prime} \in\left(E_{1}^{\prime}\right)_{\pi\left(x_{1}\right)}=\left(\rho^{\prime}\right)^{-1}\left(T_{\pi\left(x_{1}\right)} Q_{1}^{\prime}\right)$. Then, we can choose $e \in E_{x_{1}}$ such that $\Pi(e)=e_{1}^{\prime}$. Thus, from (3.10), we have that

$$
(T \pi)(\rho(e)) \in T_{\pi\left(x_{1}\right)} Q_{1}^{\prime} .
$$

Now, using that $\pi_{1}: Q_{1} \rightarrow Q_{1}^{\prime}$ is a submersion, we deduce that there exists $v_{1} \in T_{x_{1}} Q_{1}$ such that

$$
(T \pi)\left(v_{1}\right)=(T \pi)(\rho(e))
$$

that is,

$$
v_{1}-\rho(e) \in T_{x_{1}}\left(\pi^{-1}\left(\pi\left(x_{1}\right)\right)\right) \subseteq T_{x_{1}} Q_{1} .
$$

Therefore, $\rho(e) \in T_{x_{1}} Q_{1}$ and

$$
e \in \rho^{-1}\left(T_{x_{1}} Q_{1}\right)=\left(E_{1}\right)_{x_{1}} .
$$

On the other hand, if $e \in \operatorname{Ker}\left(\Pi_{\mid E_{x_{1}}}\right)$, then

$$
0=\Pi(e) \in\left(E_{1}^{\prime}\right)_{\pi\left(x_{1}\right)},
$$

and, proceeding as above, we conclude that $e \in\left(E_{1}\right)_{x_{1}}$.

Finally, using that the pair $(\Pi, \pi)$ is a dynamical Lie algebroid epimorphism, we obtain that the pair $\left(\Pi_{1}, \pi_{1}\right)$ is also a dynamical Lie algebroid epimorphism.

Next, we will prove the following theorem.

Theorem 3.3. Let $(\Pi, \pi)$ be a dynamical Lie algebroid epimorphism between $E$ and $E^{\prime}$. Then, we have that:

(i) $\pi\left(Q_{k}\right)=Q_{k}^{\prime}$ and $\Pi\left(E_{k}\right)=E_{k}^{\prime}$, for all $k$.

(ii) If $x_{k} \in Q_{k}$, then $\pi^{-1}\left(\pi\left(x_{k}\right)\right) \subseteq Q_{k}$ and $\operatorname{Ker}\left(\Pi_{\mid E_{x_{k}}}\right) \subseteq\left(E_{k}\right)_{x_{k}}$, for all $k$.

(iii) If $\pi_{k}: Q_{k} \rightarrow Q_{k}^{\prime}$ and $\Pi_{k}: E_{k} \rightarrow E_{k}^{\prime}$ are the restrictions to $Q_{k}$ and $E_{k}$ of $\pi: Q \rightarrow Q^{\prime}$ and $\Pi: E \rightarrow E^{\prime}$, respectively, then the pair $\left(\Pi_{k}, \pi_{k}\right)$ is a dynamical Lie algebroid epimorphism, for all $k$.

Proof. The result holds for $k=0,1$. Then, we will proceed by induction.

Assume that the result holds for $k \in\{0,1, \ldots, N\}$. Then, we will prove it for $k=N+1$.

Note that if $k \in\{0,1, \ldots, N\}$ and $x_{k} \in Q_{k}$ then, using the following facts

$$
(\Pi, \pi)^{*} \Omega^{\prime}=\Omega, \Pi\left(\left(E_{k}\right)_{x_{k}}\right)=\left(E_{k}^{\prime}\right)_{\pi\left(x_{k}\right)} \text { and } \Pi\left(E_{x_{k}}\right)=E_{\pi\left(x_{k}\right)}^{\prime},
$$

we obtain that

$$
\Pi\left(\left(E_{k}\right)_{x_{k}}^{\perp}\right)=\left(E_{k}^{\prime}\right)_{\pi\left(x_{k}\right)}^{\perp} .
$$

Thus, proceeding as in the proof of Lemma 3.2 , we deduce the result.

We remark that the behavior of the two constraint algorithms is the same. In fact, if we obtain a final Lie subalgebroid $\tau_{f}=\tau_{k}: E_{f}=E_{k} \rightarrow Q_{f}=Q_{k}$ for the first problem (that is, if $Q_{k}=Q_{k+1}$ ) then, from Theorem 3.3, it follows that $Q_{k}^{\prime}=Q_{k+1}^{\prime}$ and we have a final Lie subalgebroid $\tau_{f}^{\prime}=\tau_{k}^{\prime}: E_{f}^{\prime}=E_{k}^{\prime} \rightarrow Q_{f}^{\prime}=Q_{k}^{\prime}$ for the second problem. Conversely, if the second constraint algorithm stops at a certain $k$ (that 
is, $Q_{k}^{\prime}=Q_{k+1}^{\prime}$ ) then, using (3.6) and (3.11), we deduce that $Q_{k}=Q_{k+1}$, i.e., the first constraint algorithm also stops at the level $k$.

Now, suppose that $X: Q_{k} \rightarrow E_{k}$ is a section of the Lie algebroid $\tau_{k}: E_{k} \rightarrow Q_{k}$ such that

$$
i_{X} \Omega_{\mid Q_{k}}=\alpha_{\mid Q_{k}}
$$

and $X$ is $\left(\Pi_{k}, \pi_{k}\right)$-projectable, i.e., there exists $X^{\prime} \in \Gamma\left(E_{k}^{\prime}\right)$ satisfying

$$
X^{\prime} \circ \pi_{k}=\Pi_{k} \circ X .
$$

Then, using that $(\Pi, \pi)^{*} \Omega^{\prime}=\Omega$ and that $(\Pi, \pi)^{*} \alpha^{\prime}=\alpha$, we obtain that

$$
i_{X^{\prime}} \Omega_{\mid Q_{k}^{\prime}}^{\prime}=\alpha_{\mid Q_{k}^{\prime}}^{\prime}
$$

In others words, $X^{\prime}$ is a solution (along $Q_{k}^{\prime}$ ) of the second dynamical problem.

Conversely, if $X^{\prime} \in \Gamma\left(E_{k}^{\prime}\right)$ is a solution of the dynamical equation

$$
i_{X^{\prime}} \Omega_{\mid Q_{k}^{\prime}}^{\prime}=\alpha_{\mid Q_{k}^{\prime}}^{\prime}
$$

then we can choose $X \in \Gamma\left(E_{k}\right)$ such that

$$
X^{\prime} \circ \pi_{k}=\Pi_{k} \circ X
$$

and, since $(\Pi, \pi)^{*} \Omega^{\prime}=\Omega$ and $(\Pi, \pi)^{*} \alpha^{\prime}=\alpha$, we conclude that

$$
i_{X} \Omega_{\mid Q_{k}}=\alpha_{\mid Q_{k}}
$$

\section{Singular Lagrangian Systems on Lie ALGEBroids}

Let $L: E \rightarrow \mathbb{R}$ be a Lagrangian function on a Lie algebroid $\tau: E \rightarrow Q$.

Denote by $\omega_{L}$ and $E_{L}$ the Cartan 2-section and the Lagrangian energy, respectively, associated with $L$. Then, $\omega_{L}$ is not, in general, a symplectic section and, thus, the dynamical equation

$$
i_{X} \omega_{L}=d^{\mathcal{T}^{E}}{ }^{E} E_{L}
$$

has not, in general, solution. Moreover, if there exists a solution of the above equation, it is not, in general, a second order differential equation and it is not, in general, unique.

Note that the Legendre transformation $\operatorname{leg}_{L}: E \rightarrow E^{*}$ associated with $L$ is not, in general, a local diffeomorphism.

Definition 4.1. The Lagrangian function $L$ is said to be almost regular if the following conditions hold:

i) The subset $M_{1}=\operatorname{leg}_{L}(E)$ of $E^{*}$ is an embedded submanifold of $E^{*}$.

ii) The map leg $\log _{1}: M_{1}$ induced by the Legendre transformation is a submersion with connected fibres.

In what follows, we will assume that $L$ is an almost regular Lagrangian.

Then, we may prove the following result.

Proposition 4.2. The Lagrangian energy $E_{L}$ is a basic function with respect to the submersion $\operatorname{leg}_{1}: E \rightarrow M_{1}$, that is, there exists a Hamiltonian function $H$ on $M_{1}$ such that

$$
H \circ \operatorname{leg}_{1}=E_{L} \text {. }
$$


Proof. Suppose that $e$ is a point of $E$ and that $\left(x^{i}, y^{A}\right)$ are fibred local coordinates in an open subset of $E$ which contains to $e$. Then, using (2.11), we deduce that $X=\lambda^{A} \frac{\partial}{\partial y^{A} \mid e} \in T_{e} E$ is vertical with respect to the submersion $\operatorname{leg}_{1}: E \rightarrow M_{1}$ if and only if

$$
\lambda^{A} \frac{\partial^{2} L}{\partial y^{A} \partial y^{B}}=0, \text { for all } B .
$$

Thus, if $X$ is vertical, from (2.7), it follows that

$$
X\left(E_{L}\right)=\lambda^{A}{\frac{\partial L}{\partial y^{A}}}_{\mid e}+\lambda^{A} y^{B}(e) \frac{\partial^{2} L}{\partial y^{A} \partial y_{\mid e}^{B}}-\lambda^{A}{\frac{\partial L}{\partial y_{\mid e}^{A}}}_{\mid e}=0 .
$$

This ends the proof of the result.

Now, since $\tau_{\mid M_{1}}^{*}: M_{1} \rightarrow Q$ is a fibration, one may consider the prolongation $\mathcal{T}^{E} M_{1}$ of the Lie algebroid $\tau: E \rightarrow Q$ over $\tau_{\mid M_{1}}^{*}$ or, in other words, the $E$-tangent bundle to $M_{1}$.

Then, the canonical symplectic section $\Omega_{E}$ on $\mathcal{T}^{E} E^{*} \rightarrow E^{*}$ induces a presymplectic section $\Omega_{1}$ on the Lie algebroid $\mathcal{T}^{E} M_{1} \rightarrow M_{1}$. Moreover, the submersion $\operatorname{leg}_{1}: E \rightarrow M_{1}$ induces, in a natural way, a Lie algebroid epimorphism

$$
\mathcal{T} \operatorname{leg}_{1}: \mathcal{T}^{E} E \rightarrow \mathcal{T}^{E} M_{1}
$$

(over $l e g_{1}$ ). In addition, using (2.12) and Proposition 4.2. we obtain that

$$
\left(\mathcal{T} \text { leg }_{1}, \text { leg }_{1}\right)^{*}\left(\Omega_{1}\right)=\omega_{L}, \quad\left(\mathcal{T} \text { leg }_{1}, \text { leg }_{1}\right)^{*}\left(d^{\mathcal{T}^{E} E} E_{L}\right)=d^{\mathcal{T}^{E} M_{1}} H
$$

Therefore, we have proved the following result.

Proposition 4.3. The pair $\left(\mathcal{T} l_{\text {eg }}, l_{\text {eg }}\right)$ is a dynamical Lie algebroid epimorphism between the presymplectic Lie algebroids $\left(\mathcal{T}^{E} E, \omega_{L}, d^{\mathcal{T}^{E} E} E_{L}\right)$ and $\left(\mathcal{T}^{E} M_{1}, \Omega_{1}\right.$, $\left.d^{\mathcal{T}^{E} M_{1}} H\right)$.

The following diagram illustrates the above situation.

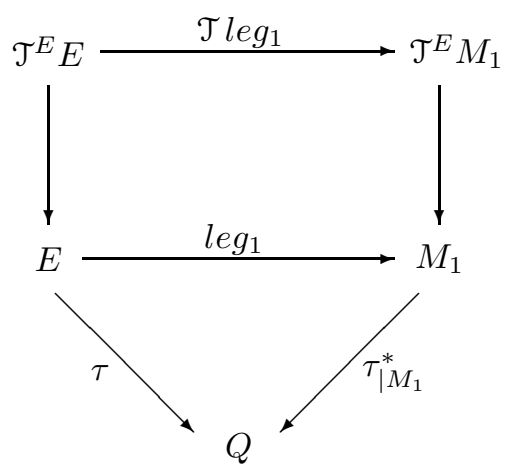

Now, we consider the following dynamical equations

$$
i_{X} \omega_{L}=d^{\mathcal{T}^{E} E} E_{L}, \quad \text { with } X \in \Gamma\left(\mathcal{T}^{E} E\right)
$$

and

$$
i_{Y} \Omega_{1}=d^{\mathcal{T}^{E} M_{1}} H, \quad \text { with } Y \in \Gamma\left(\mathcal{T}^{E} M_{1}\right) .
$$

In general, a section $X \in \Gamma\left(\mathcal{T}^{E} E\right)$ (respectively, $Y \in \Gamma\left(\mathcal{T}^{E} M_{1}\right)$ ) satisfying (4.1) (respectively, (4.2) ) can not be found in all the points of $E$ (respectively, $M_{1}$ ). Thus, we must apply the general constraint algorithm developed in Section 3.1 for an arbitrary presymplectic system. 
Assume that this algorithm stops at the level $k$ for the first dynamical equation, that is, there exists a Lie subalgebroid $\left(\mathcal{T}^{E} E\right)_{k}$ of $\mathcal{T}^{E} E$ over a submanifold $E_{k}$ of $E$ and a section $X_{k} \in \Gamma\left(\left(\mathcal{T}^{E} E\right)_{k}\right)$ such that

$$
\left(i_{X_{k}} \omega_{L}\right)_{\mid E_{k}}=\left(d^{\mathcal{T}^{E}} E^{E} E_{L}\right)_{\mid E_{k}}
$$

Note that $\left(\mathcal{T}^{E} E\right)_{k}=\left(\rho^{\tau}\right)^{-1}\left(T E_{k}\right)$, where $\rho^{\tau}: \mathcal{T}^{E} E \rightarrow T E$ is the anchor map of the Lie algebroid $\mathcal{T}^{E} E \rightarrow E$. Moreover, using Proposition 4.3 and the results of Section 3.2. we deduce that the constraint algorithm also stops at the level $k$ for the second equation. In fact, we have that:

(i) $\operatorname{leg}_{1}\left(E_{k}\right)=M_{k+1}$ is a submanifold of $M_{1}$ and

$$
\left(\mathcal{T}^{E} M_{1}\right)_{k}=\left(\mathcal{T} \operatorname{leg}_{1}\right)\left(\left(\mathcal{T}^{E} E\right)_{k}\right)=\left(\rho^{\tau^{*}}\right)^{-1}\left(T M_{k+1}\right)
$$

is a Lie algebroid over $M_{k+1}, \rho^{\tau^{*}}: \mathcal{T}^{E} E^{*} \rightarrow T E^{*}$ being the anchor map of the Lie algebroid $\mathcal{T}^{E} E^{*} \rightarrow E^{*}$.

(ii) If $e_{k} \in E_{k}$ then $l e g_{1}^{-1}\left(\operatorname{leg}_{1}\left(e_{k}\right)\right) \subseteq E_{k}$ and $\operatorname{Ker}\left(\mathcal{T}_{e_{k}} l e g_{1}\right) \subseteq\left(\mathcal{T}_{e_{k}}^{E} E\right)_{k}$. Note that, from (2.6) and (2.11), it follows that

$$
\operatorname{Ker}\left(\mathcal{T}_{e_{k}} \operatorname{leg}_{1}\right)=\operatorname{Ker} \omega_{L}\left(e_{k}\right) \cap\left(\mathcal{T}_{e_{k}}^{E} E\right)^{V} .
$$

(iii) If $\operatorname{leg}_{k+1}: E_{k} \rightarrow M_{k+1}$ and $\operatorname{Leg}_{k+1}:\left(\mathcal{T}^{E} E\right)_{k} \rightarrow\left(\mathcal{T}^{E} M_{1}\right)_{k}$ are the restrictions to $E_{k}$ and $\left(\mathcal{T}^{E} E\right)_{k}$ of $\operatorname{leg}_{1}: E=E_{0} \rightarrow M_{1}$ and $\mathcal{T} \operatorname{leg}_{1}: \mathcal{T}^{E} E \rightarrow$ $\mathcal{T}^{E} M_{1}$, respectively, then the pair $\left(\operatorname{Leg}_{k+1}, \operatorname{leg}_{k+1}\right)$ is a dynamical Lie algebroid epimorphism.

(iv) If $X_{k} \in \Gamma\left(\left(\mathcal{T}^{E} E\right)_{k}\right)$ is such that $\left(i_{X_{k}} \omega_{L}\right)_{\mid E_{k}}=\left(d^{\mathcal{T}^{E} E} E_{L}\right)_{\mid E_{k}}$ and $X_{k}$ is $\left(\operatorname{Leg}_{k+1}, \operatorname{leg}_{k+1}\right)$-projectable, i.e., there exists $Y_{k} \in \Gamma\left(\left(\mathcal{T}^{E} M_{1}\right)_{k}\right)$ satisfying

$$
Y_{k} \circ \operatorname{leg}_{k+1}=\operatorname{Leg}_{k+1} \circ X_{k},
$$

then

$$
\left(i_{Y_{k}} \Omega_{1}\right)_{\mid M_{k+1}}=\left(d^{\mathcal{T}^{E} M_{1}} H\right)_{\mid M_{k+1}} .
$$

(v) If $Y_{k} \in \Gamma\left(\left(\mathcal{T}^{E} M_{1}\right)_{k}\right)$ is a solution of the dynamical equation

$$
\left(i_{Y_{k}} \Omega_{1}\right)_{\mid M_{k+1}}=\left(d^{\mathcal{T}^{E} M_{1}} H\right)_{\mid M_{k+1}}
$$

then we can choose $X_{k} \in \Gamma\left(\left(\mathcal{T}^{E} E\right)_{k}\right)$ such that

$$
Y_{k} \circ \operatorname{leg}_{k+1}=\operatorname{Leg}_{k+1} \circ X_{k} \text { and }\left(i_{X_{k}} \omega_{L}\right)_{\mid E_{k}}=\left(d^{\mathcal{T}^{E} E} E_{L}\right)_{\mid E_{k}}
$$

Now, suppose that $X \in \Gamma\left(\left(\mathcal{T}^{E} E\right)_{k}\right)$ is a solution of the dynamical equation

$$
\left(i_{X} \omega_{L}\right)_{\mid E_{k}}=\left(d^{\mathcal{T}^{E}} E^{E} E_{\mid E_{k}}\right.
$$

and that $X$ is $\left(\operatorname{Leg}_{k+1}, \operatorname{leg}_{k+1}\right)$-projectable over $Y \in \Gamma\left(\left(\mathcal{T}^{E} M_{1}\right)_{k}\right)$.

Then,

$$
\left(i_{Y} \Omega_{1}\right)_{\mid M_{k+1}}=\left(d^{\mathcal{T}^{E}} M_{1} H\right)_{\mid M_{k+1}} .
$$

The following diagram illustrates the above situation. 


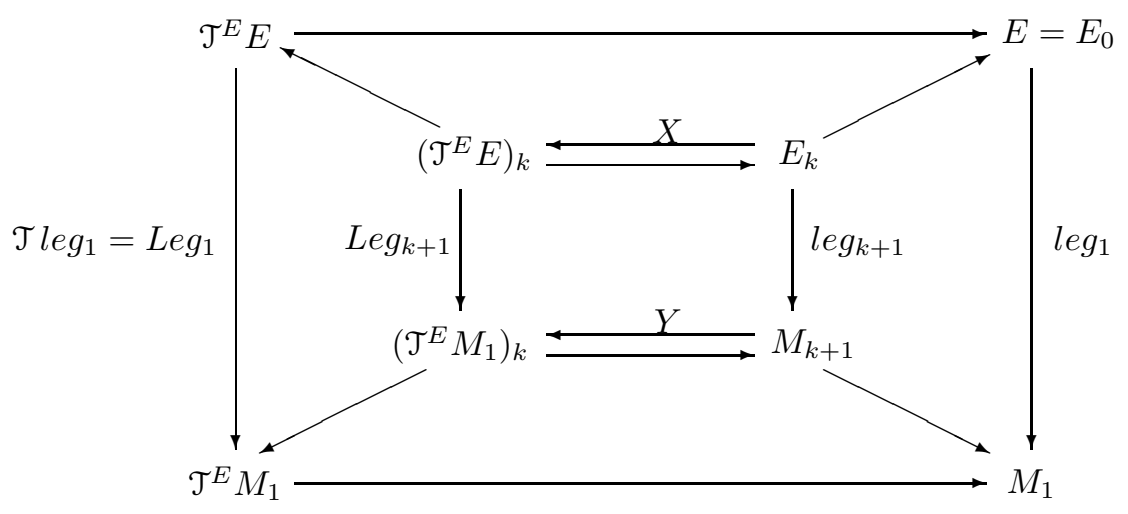

If $\rho_{k}^{\tau}:\left(\mathcal{T}^{E} E\right)_{k} \rightarrow T E_{k}$ is the anchor map of the Lie algebroid $\left(\mathcal{T}^{E} E\right)_{k} \rightarrow E_{k}$ then the integral curves of the vector field $\rho_{k}^{\tau}(X)$ don't satisfy, in general, the EulerLagrange equations for $L$. The reason is that the section $X$ is not, in general, a SODE along the submanifold $E_{k}$ of $E$. In other words, $X$ doesn't satisfy, in general, the equation

$$
S X=\Delta_{\mid E_{k}} .
$$

A solution for the above problem is given in the following theorems.

Theorem 4.4. (i) The subset $S^{X}$ of $E_{k}$ defined by

$$
S^{X}=\left\{e \in E_{k} /(S X)(e)=\Delta(e)\right\}
$$

is a submanifold of $E_{k}$.

(ii) There exists a Lie subalgebroid $A^{X}$ of $\left(\mathcal{T}^{E} E\right)_{k} \rightarrow E_{k}$ (over $S^{X}$ ) such that if $\operatorname{Leg}_{A^{x}}: A^{X} \rightarrow\left(\mathcal{T}^{E} M_{1}\right)_{k}$ and $\operatorname{leg}_{S^{X}}: S^{X} \rightarrow M_{k+1}$ are the restrictions of $\operatorname{Leg}_{k+1}$ : $\left(\mathcal{T}^{E} E\right)_{k} \rightarrow\left(\mathcal{T}^{E} M_{1}\right)_{k}$ and $\operatorname{leg}_{k+1}: E_{k} \rightarrow M_{k+1}$ to $A^{X}$ and $S^{X}$, respectively, then the pair $\left(\operatorname{Leg}_{A^{x}}, \operatorname{leg}_{S^{x}}\right)$ is a Lie algebroid isomorphism.

Theorem 4.5. There is a unique section $\xi^{X} \in \Gamma\left(A^{X}\right)$ satisfying the following conditions

$$
\left(i_{\xi^{X}} \omega_{L}\right)_{\mid S^{X}}=\left(d^{\mathcal{T}^{E} E} E_{L}\right)_{\mid S^{X}}, \quad S\left(\xi^{X}\right)=\Delta_{\mid S^{X}} .
$$

Theorem 4.6. If $\rho_{A^{X}}: A^{X} \rightarrow T S^{X}$ is the anchor map of the Lie algebroid $A^{X} \rightarrow$ $S^{X}$ then the integral curves of the vector field $\rho_{A^{X}}\left(\xi^{X}\right)$ on $S^{X}$ are solutions of the Euler-Lagrange equations for $L$.

Proof of Theorem 4.4 We consider the smooth map $W_{X}: E_{k} \rightarrow E$ defined by

$$
W_{X}(e)=p r_{1}(X(e)),
$$

where $p r_{1}: \mathcal{T}^{E} E \rightarrow E$ is the restriction to $\mathcal{T}^{E} E$ of the canonical projection $p r_{1}$ : $E \times T E \rightarrow E$ on the first factor.

Now, we will proceed in several steps.

First step: We will prove that

$$
S^{X} \cap \operatorname{leg}_{k+1}^{-1}\left(\operatorname{leg}_{k+1}(e)\right)=\left\{W_{X}(e)\right\} \text {, for all } e \in E_{k} .
$$

If $e \in E_{k}$ then, using (2.3), (2.6), (2.7) and the fact that

we deduce that

$$
\left(i_{X} \omega_{L}\right)_{\mid E_{k}}=\left(d^{\mathcal{T}^{E} E} E_{L}\right)_{\mid E_{k}},
$$

$$
(S X-\Delta)(e) \in \operatorname{Ker\omega }_{L}(e) .
$$


On the other hand, it is clear that

$$
(S X-\Delta)(e) \in\left(\mathcal{T}_{e}^{E} E\right)^{V} .
$$

Thus, from (4.3), we obtain that

$$
(S X-\Delta)(e) \in \operatorname{Ker}\left(\mathcal{T}_{e} l_{e} g_{1}\right) .
$$

Therefore, if $\rho^{\tau}: \mathcal{T}^{E} E \rightarrow T E$ is the anchor map of the Lie algebroid $\mathcal{T}^{E} E$, then

$$
\rho^{\tau}((S X-\Delta)(e)) \in \operatorname{Ker}\left(T_{e} l_{e} g_{1}\right)=\operatorname{Ker}\left(T_{e} l_{e} g_{k+1}\right) .
$$

This implies that $X^{*}=\rho^{\tau}\left(S X-\Delta_{\mid E_{k}}\right)$ is a vector field on $E_{k}$ which is vertical with respect to the submersion $\operatorname{leg}_{k+1}: E_{k} \rightarrow M_{k+1}$.

Suppose that the local expression of the section $X$ is

$$
X=X^{A} x_{A}+V^{A} \nu_{A} .
$$

Then,

$$
S X-\Delta=\left(X^{A}-y^{A}\right) \mathcal{V}_{A} \quad \text { and } \quad X^{*}=\left(X^{A}-y^{A}\right) \frac{\partial}{\partial y^{A}} .
$$

Moreover, since $X$ is $\left(\operatorname{Leg}_{k+1}, \operatorname{leg}_{k+1}\right)$-projectable, the functions $X^{A}$ are constant on the fibres of $\operatorname{leg}_{k+1}$. Consequently, if $e \equiv\left(x_{0}^{i}, y_{0}^{A}\right) \in E_{k}$, it follows that the integral curve of $X^{*}$ over the point $e$ is

$$
s \mapsto \sigma(s) \equiv\left(x_{0}^{i}, X^{A}+e^{-s}\left(y_{0}^{A}-X^{A}\right)\right) .
$$

In particular,

$$
\sigma(s) \equiv\left(x_{0}^{i}, X^{A}+e^{-s}\left(y_{0}^{A}-X^{A}\right)\right) \in \operatorname{leg}_{k+1}^{-1}\left(\operatorname{leg}_{k+1}(e)\right), \text { for all } s \in \mathbb{R},
$$

which implies that

$$
\lim _{s \rightarrow \infty} \sigma(s) \equiv\left(x_{0}^{i}, X^{A}\right) \in \operatorname{leg}_{k+1}^{-1}\left(\operatorname{leg}_{k+1}(e)\right) .
$$

Now, from (4.6), we have that

$$
W_{X}(e)=\operatorname{pr}_{1}(X(e)) \equiv\left(x_{0}^{i}, X^{A}\right) .
$$

In addition, using (4.7) and (4.8), one deduces that

$$
X^{*}\left(W_{X}(e)\right)=0 .
$$

On the other hand, if $e^{\prime} \in S^{X} \cap \operatorname{leg}_{k+1}^{-1}\left(\operatorname{leg}_{k+1}(e)\right)$ then, it is clear that

$$
\tau(e)=\tau\left(e^{\prime}\right)
$$

and, thus, $e^{\prime} \equiv\left(x_{0}^{i}, y^{A}\left(e^{\prime}\right)\right)$. Furthermore, since $e^{\prime} \in S^{X}$, we obtain that

$$
0=X^{*}\left(e^{\prime}\right)=\left(X^{A}-y^{A}\left(e^{\prime}\right)\right) \frac{\partial}{\partial y^{A}}{ }_{\mid e^{\prime}}
$$

which implies that

$$
e^{\prime} \equiv\left(x_{0}^{i}, X^{A}\right) \equiv W_{X}(e) .
$$

Second step: We will prove that

$$
S^{X}=\widetilde{W}_{X}\left(M_{k+1}\right),
$$

where $\widetilde{W}_{X}: M_{k+1} \rightarrow E_{k}$ is a section of the submersion $\operatorname{leg}_{k+1}: E_{k} \rightarrow M_{k+1}$. Therefore, $S^{X}$ is a submanifold of $E_{k}$ and $\operatorname{dim} S^{X}=\operatorname{dim} M_{k+1}$.

In fact, suppose that $e, e^{\prime} \in E_{k}$ and

$$
\operatorname{leg}_{k+1}(e)=\operatorname{leg}_{k+1}\left(e^{\prime}\right) \text {. }
$$

Then,

$$
\operatorname{Leg}_{k+1}(X(e))=Y\left(\operatorname{leg}_{k+1}(e)\right)=Y\left(\operatorname{leg}_{k+1}\left(e^{\prime}\right)\right)=\operatorname{Leg}_{k+1}\left(X\left(e^{\prime}\right)\right)
$$


Consequently,

$$
W_{X}(e)=p r_{1}(X(e))=p r_{1}\left(X\left(e^{\prime}\right)\right)=W_{X}\left(e^{\prime}\right) .
$$

So, we have that there exists a smooth map $\widetilde{W}_{X}: M_{k+1} \rightarrow E_{k}$ such that the following diagram is commutative

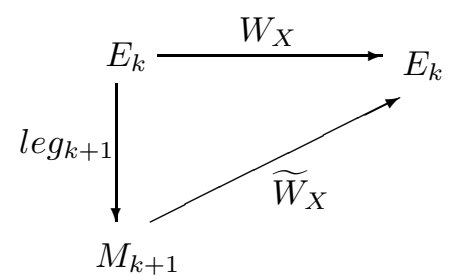

Moreover, using (4.5), we deduce that $\widetilde{W}_{X}: M_{k+1} \rightarrow E_{k}$ is a section of the submersion $\operatorname{leg}_{k+1}: E_{k} \rightarrow M_{k+1}$ and

$$
S^{X}=\widetilde{W}_{X}\left(M_{k+1}\right) .
$$

Third step: We will prove the second part of the theorem.

The section $\widetilde{W}_{X}: M_{k+1} \rightarrow E_{k}$ induces a map

$$
\mathcal{T} \widetilde{W}_{X}:\left(\mathcal{T}^{E} M_{1}\right)_{k}=\left(\rho^{\tau^{*}}\right)^{-1}\left(T M_{k+1}\right) \rightarrow\left(\mathcal{T}^{E} E\right)_{k}=\left(\rho^{\tau}\right)^{-1}\left(T E_{k}\right)
$$

in such a way that the pair $\left(\mathcal{T} \widetilde{W}_{X}, \widetilde{W}_{X}\right)$ is a Lie algebroid monomorphism. We will denote by $A^{X}$ the image of $\left(\mathcal{T}^{E} M_{1}\right)_{k}$ by the map $\mathcal{T} \widetilde{W}_{X}$. Then, it is clear that $A^{X}$ is a Lie subalgebroid (over $S^{X}$ ) and the pair $\left(\mathcal{T} \widetilde{W}_{X}, \widetilde{W}_{X}\right)$ is an isomorphism between the Lie algebroids $A^{X} \rightarrow S^{X}$ and $\left(\mathcal{T}^{E} M_{1}\right)_{k} \rightarrow M_{k+1}$. In fact, the inverse morphism is the pair $\left(\operatorname{Leg}_{A^{X}}, \operatorname{leg}_{S^{X}}\right)$.

Proof of Theorem 4.5. We consider the section $\xi^{X} \in \Gamma\left(A^{X}\right)$ defined by

$$
\xi^{X}=\mathcal{T} \widetilde{W}_{X} \circ Y \circ \operatorname{leg}_{S^{x}} .
$$

Using (4.4) and the fact that

$$
\operatorname{Leg}_{A^{X}} \circ \xi^{X}=Y \circ \operatorname{leg}_{S^{X}}
$$

it follows that

$$
\left(i_{\xi^{X}} \omega_{L}\right)_{\mid S^{X}}=\left(d^{\mathcal{T}^{E} E} E_{L}\right)_{\mid S^{X}} .
$$

Now, from (4.9), we have that

$$
\left(\xi^{X}-X\right)(e) \in \operatorname{Ker}\left(\operatorname{Leg}_{k+1}\right)_{\mid\left(\mathcal{T}_{e}^{E} E\right)_{k}}=\operatorname{Ker}\left(\mathcal{T}_{e} l e g_{1}\right), \text { for all } e \in S^{X},
$$

which implies that (see (4.3))

$$
\left(S \xi^{X}\right)(e)=(S X)(e)=\Delta(e), \text { for all } e \in S^{X} .
$$

Next, suppose that $\eta$ is another section of the Lie algebroid $A^{X} \rightarrow S^{X}$ such that

$$
\left(i_{\eta} \omega_{L}\right)_{\mid S^{X}}=\left(d^{\mathcal{T}^{E} E} E_{L}\right)_{\mid S^{X}}, \quad S \eta=\Delta_{\mid S^{X}} .
$$

Then, it is clear that

$$
\left(\eta-\xi^{X}\right)(e) \in \operatorname{Ker}_{L}(e) \cap\left(\mathcal{T}_{e}^{E} E\right)^{V}, \text { for all } e \in S^{X},
$$

and, using (4.3), we deduce that

$$
\left(\eta-\xi^{X}\right)(e) \in \operatorname{Ker}\left(\mathcal{T}_{e} \operatorname{leg}_{1}\right)=\operatorname{Ker}\left(\operatorname{Leg}_{k+1}\right)_{\mid\left(\mathcal{T}_{e}^{E} E\right)_{k}}, \text { for all } e \in S^{X} .
$$

Thus,

$$
\operatorname{Leg}_{A^{X}}\left(\left(\eta-\xi^{X}\right)(e)\right)=0
$$


and, since $\operatorname{Leg}_{A^{X}}: A^{X} \rightarrow\left(\mathcal{T}^{E} M_{1}\right)_{k}$ is a vector bundle isomorphism, we conclude that

$$
\eta=\xi^{X}
$$

Proof of Theorem 4.6. The section $\xi^{X}$ is a SODE along the submanifold $S^{X}$. Therefore, from (2.3) and (2.4), we have that the local expression of $\xi^{X}$ is

$$
\xi^{X}=y^{B} x_{B \mid S^{X}}+\xi^{B} \mathcal{V}_{B \mid S^{X}} .
$$

On the other hand, using (2.6), (2.7), (4.10) and the fact that $\left(i_{\xi^{x}} \omega_{L}\right)_{\mid S^{X}}=$ $\left(d^{\mathcal{T}^{E} E} E_{L}\right)_{\mid S^{X}}$, it follows that

$$
\xi^{B} \frac{\partial^{2} L}{\partial y^{A} \partial y^{B}}+y^{B} \rho_{B}^{i} \frac{\partial^{2} L}{\partial x^{i} \partial y^{A}}+\frac{\partial L}{\partial y^{C}} \bigodot_{A B}^{C} y^{B}-\rho_{A}^{i} \frac{\partial L}{\partial x^{i}}=0, \text { for all } A \text {. }
$$

Now, the local expression of the vector field $\rho_{A^{X}}\left(\xi^{X}\right)$ on $S^{X}$ is

$$
\rho_{A^{X}}\left(\xi^{X}\right)=\rho_{B}^{i} y^{B} \frac{\partial}{\partial x^{i}}+\xi^{B} \frac{\partial}{\partial y^{B}} .
$$

Consequently, the integral curves of $\rho_{A^{x}}\left(\xi^{X}\right)$ satisfy the following equations

$$
\frac{d x^{i}}{d t}=\rho_{A}^{i} y^{A}, \quad \frac{d}{d t}\left(\frac{\partial L}{\partial y^{A}}\right)+\frac{\partial L}{\partial y^{C}} \varrho_{A B}^{C} y^{B}-\rho_{A}^{i} \frac{\partial L}{\partial x^{i}}=0, \text { for all } i \text { and } A,
$$

which are the Euler-Lagrange equations for $L$.

Remark 4.7. If we apply the results obtained in this Section for the particular case when the Lie algebroid $E$ is $T Q$, we recover the results proved in [11, 12 , for standard singular Lagrangian systems.

Example 4.8. To illustrate the theory we will consider a variation of an example of singular lagrangian with symmetry. This example corresponds to a mechanical model of field theories due to Capri and Kobayashi (see [6, 7]).

Consider the lagrangian function

$$
L=\frac{1}{2} m_{2}\left(\dot{x}_{2}^{2}+\dot{y}_{2}^{2}\right)+\dot{y}_{2} x_{2}-\dot{x}_{2} y_{2}-x_{1}^{2}-y_{1}^{2}-x_{2}^{2}-y_{2}^{2} .
$$

The configuration space is $\widetilde{Q}=\mathbb{R}^{4}$ with local coordinates $\left(x_{1}, y_{1}, x_{2}, y_{2}\right)$. Clearly, the lagrangian is singular; in fact, since

$$
\omega_{L}=m_{2} d x_{2} \wedge d \dot{x}_{2}+m_{2} d y_{2} \wedge d \dot{y}_{2}+2 d y_{2} \wedge d x_{2},
$$

then

$$
\operatorname{Ker} \omega_{L}=\operatorname{span}\left\{\frac{\partial}{\partial x_{1}}, \frac{\partial}{\partial y_{1}}, \frac{\partial}{\partial \dot{x}_{1}}, \frac{\partial}{\partial \dot{y}_{1}}\right\} \text {. }
$$

The system is invariant by the $S^{1}$ action

$$
\begin{aligned}
S^{1} \times \widetilde{Q} & \longrightarrow \widetilde{Q} \\
\left(\alpha,\left(x_{1}, y_{1}, x_{2}, y_{2}\right)\right) & \longmapsto\left(x_{1}, y_{1}, x_{2} \cos \alpha-y_{2} \sin \alpha, x_{2} \sin \alpha+y_{2} \cos \alpha\right)
\end{aligned}
$$

Note that the action of $S^{1}$ on the open subset $Q=\mathbb{R}^{2} \times\left(\mathbb{R}^{2}-\{(0,0)\}\right)$ is free and then we may consider the reduced space $Q / S^{1}$ and the Atiyah algebroid $\tau_{Q} \mid S^{1}$ : $T Q / S^{1} \rightarrow Q / S^{1}$. Taking polar coordinates $x_{2}=\rho \cos \theta$ and $y_{2}=\rho \sin \theta$, we have that the canonical projection $\pi: Q \rightarrow M=Q / S^{1}$ is given by

$$
\pi\left(x_{1}, y_{1}, \rho, \theta\right)=\left(x_{1}, y_{1}, \rho\right) .
$$


It is clear that the Atiyah algebroid is isomorphic to the vector bundle $T M \times \mathbb{R} \rightarrow$ $M$.

On the other hand, a local basis of $S^{1}$-invariant vector fields on $Q$ is $\left\{\frac{\partial}{\partial x_{1}}, \frac{\partial}{\partial y_{1}}, \frac{\partial}{\partial \rho}\right.$, $\left.\frac{\partial}{\partial \theta}\right\}$. These vector fields induce a local basis of sections $\left\{e_{1}, e_{2}, e_{3}, e_{0}\right\}$ of the Atiyah algebroid $\tau_{Q} \mid S^{1}: T Q / S^{1} \rightarrow M=Q / S^{1}$. Moreover, if $(\llbracket \cdot, \cdot \rrbracket, \rho)$ is the Lie algebroid structure on $\tau_{Q} \mid S^{1}: T Q / S^{1} \rightarrow M=Q / S^{1}$, it follows that $\llbracket e_{i}, e_{j} \rrbracket=0$, for all $i$ and $j$, and

$$
\rho\left(e_{1}\right)=\frac{\partial}{\partial x_{1}}, \quad \rho\left(e_{2}\right)=\frac{\partial}{\partial y_{1}}, \quad \rho\left(e_{3}\right)=\frac{\partial}{\partial \rho}, \quad \rho\left(e_{0}\right)=0 .
$$

Now, if $\left(x_{1}, y_{1}, \rho, \dot{x}_{1}, \dot{y}_{1}, \dot{\rho}, r\right)$ are the local coordinates on $T Q / S^{1}$ induced by the local basis $\left\{e_{1}, e_{2}, e_{3}, e_{0}\right\}$, then the reduced lagrangian is

$$
l=\frac{1}{2} m_{2}\left(\dot{\rho}^{2}+(\rho r)^{2}\right)+\rho^{2} r-x_{1}^{2}-y_{1}^{2}-\rho^{2} .
$$

Thus, the Euler-Lagrange equations for $l$ are:

$$
\begin{aligned}
m_{2} \ddot{\rho}-\left(m_{2} r+2\right) \rho r+2 \rho & =0 \\
m_{2} r \rho^{2}+\rho^{2} & =\text { constant } \\
x_{1} & =0 \\
y_{1} & =0 .
\end{aligned}
$$

The local basis $\left\{e_{1}, e_{2}, e_{3}, e_{0}\right\}$ of $\Gamma\left(T Q / S^{1}\right)$ induces a local basis

$$
\left\{x_{1}, x_{2}, x_{3}, x_{0}, v_{1}, v_{2}, \nu_{3}, v_{0}\right\}
$$

of $\Gamma\left(\mathcal{T}^{T Q / S^{1}}\left(T Q / S^{1}\right)\right)$. The presymplectic 2 -section $\omega_{l}$ is written as

$$
\omega_{l}=m_{2} X^{3} \wedge \mathcal{V}^{3}+m_{2} \rho^{2} X^{0} \wedge \mathcal{V}^{0}-\rho\left(m_{2} r+2\right) X^{3} \wedge X^{0} .
$$

The energy function is

$$
E_{l}=\frac{1}{2} m_{2}\left(\dot{\rho}^{2}+(\rho r)^{2}\right)+x_{1}^{2}+y_{1}^{2}+\rho^{2}
$$

and

$$
d^{\mathcal{T}^{T Q / S^{1}} T Q / S^{1}} E_{l}=m_{2} \dot{\rho} \mathcal{V}^{3}+\rho^{2} m_{2} r \mathcal{V}^{0}+\left(m_{2} \rho r^{2}+2 \rho\right) X^{3}+2 x_{1} X^{1}+2 y_{1} X^{2} .
$$

Thus ker $\omega_{l}=\left\{X_{1}, X_{2}, \mathcal{V}_{1}, \mathcal{V}_{2}\right\}$ and the primary constraint submanifold $E_{1} \subset E=$ $E_{0}$ is determined by the vanishing of the constraints functions: $x_{1}=0, y_{1}=0$. Now $\left(\left(\rho^{\tau_{Q}} \mid S^{1}\right)^{-1}\left(T E_{1}\right)\right)^{\perp}=\operatorname{ker} \omega_{l}$, and therefore $E_{f}=E_{1}$.

Any solution $X \in\left(\mathcal{T}^{E} E\right)_{1}$ of the dynamical equation

$$
\left(i_{X} \omega_{l}\right)_{\mid E_{1}}=\left(d^{\mathcal{T}^{T Q / S^{1}} T Q / S^{1}} E_{l}\right)_{\mid E_{1}}
$$

is of the form:

$$
X=\dot{\rho} X_{3}+r X_{0}+\tilde{f} \mathcal{V}_{1}+\tilde{g} \mathcal{V}_{2}+\frac{\left(m_{2} r+2\right)-2 \rho}{m_{2}} \mathcal{V}_{3}-\frac{2 \dot{\rho}\left(m_{2} r+1\right)}{m_{2} \rho} \mathcal{V}_{0},
$$

where $\tilde{f}$ and $\tilde{g}$ are arbitrary functions on $E_{1}$.

The Legendre transformation $l e g_{l}$ is in this particular case:

$$
\operatorname{leg}_{l}\left(x_{1}, y_{1}, \rho ; \dot{x}_{1}, \dot{y}_{1}, \dot{\rho}, r\right)=\left(x_{1}, y_{1}, \rho ; 0,0, m_{2} \dot{\rho}, m_{2} \rho^{2} r+\rho^{2}\right)
$$

Therefore, the submanifold $M_{1}=\operatorname{leg}_{l}\left(T Q / S^{1}\right)$ of $T^{*} Q / S^{1}$ is defined the constraints $p_{x_{1}}=0$ and $p_{x_{2}}=0$ where we choose coordinates $\left(x_{1}, y_{1}, \rho ; p_{x_{1}}, p_{y_{1}}, p_{\rho}, p_{r}\right)$ on $T^{*} Q / S^{1}$. 
In the induced coordinates $\left(x_{1}, y_{1}, \rho ; p_{\rho}, p_{r}\right)$ on $M_{1}$, the hamiltonian $h: M_{1} \rightarrow \mathbb{R}$ is:

$$
h\left(x_{1}, y_{1}, \rho ; p_{\rho}, p_{r}\right)=\frac{1}{2 m_{2}} p_{\rho}^{2}+\frac{1}{2 m_{2} \rho^{2}}\left(p_{r}-\rho^{2}\right)^{2}+x_{1}^{2}+y_{1}^{2}+\rho^{2} .
$$

Applying the constraint algorithm to the presymplectic Lie algebroid ( $\mathcal{T}^{E} M_{1}, \Omega_{1}$, $d^{\mathcal{T}^{E}} M_{1} h$ ) we deduce that the final constraint submanifold $M_{2}$ is determined by

$$
M_{2}=\left\{\left(x_{1}, y_{1}, \rho ; p_{\rho}, p_{r}\right) \in M_{1} \mid x_{1}=0, y_{1}=0\right\}
$$

where there exists a well defined solution of

$$
\left(i_{Y} \Omega_{1}\right)_{\mid M_{2}}=\left(d^{\mathcal{T}^{E} M_{1}} h\right)_{\mid M_{2}} .
$$

Now, we return to the lagrangian picture and we study the SODE problem. Observe first that all the solutions $X$ of Equation (4.11) are ( Leg $\left._{2}, \operatorname{leg}_{2}\right)$-projectable. Now, if we additionally impose the condition $S X=\Delta_{\mid E_{1}}$, that is,

$$
\dot{\rho} \mathcal{V}_{3}+r \mathcal{V}_{0}=\dot{x}_{1} \mathcal{V}_{1}+\dot{y}_{1} \mathcal{V}_{2}+\dot{\rho} \mathcal{V}_{3}+r \mathcal{V}_{0}
$$

along $E_{1}$, we obtain that the submanifold $S^{X} \subseteq E_{1}$ is uniquely defined as

$$
S^{X}=\left\{(0,0, \rho ; 0,0, \dot{\rho}, r) \in T Q / S^{1}\right\}
$$

and, therefore, the section $\xi^{X}$ is the SODE defined by:

$$
\xi^{X}=\dot{\rho} X_{3 \mid S^{X}}+r X_{0 \mid S^{X}}+\frac{\left(m_{2} r+2\right)-2 \rho}{m_{2}} \mathcal{V}_{3 \mid S^{X}}-\frac{2 \dot{\rho}\left(m_{2} r+1\right)}{m_{2} \rho} \mathcal{V}_{0 \mid S^{X}}
$$

\section{VAKONOMIC MEChANICS ON LIE ALGEBROIDS}

5.1. Vakonomic equations and vakonomic bracket. Let $\tau: E \rightarrow Q$ be a Lie algebroid of rank $n$ over a manifold $Q$ of dimension $m$ and $L: E \rightarrow \mathbb{R}$ be a Lagrangian function on $E$. Moreover, let $M \subset E$ be an embedded submanifold of dimension $n+m-\bar{m}$ such that $\tau_{M}=\tau_{\mid M}: M \rightarrow Q$ is a surjective submersion.

Now, suppose that $e$ is a point of $M, \tau_{M}(e)=x \in Q$, that $\left(x^{i}\right)$ are local coordinates on an open subset $U$ of $Q, x \in U$, and that $\left\{e_{A}\right\}$ is a local basis of $\Gamma(E)$ on $U$. Denote by $\left(x^{i}, y^{A}\right)$ the corresponding local coordinates for $E$ on the open subset $\tau^{-1}(U)$. Assume that

$$
M \cap \tau^{-1}(U) \equiv\left\{\left(x^{i}, y^{A}\right) \in \tau^{-1}(U) \mid \Phi^{\alpha}\left(x^{i}, y^{A}\right)=0, \alpha=1, \ldots, \bar{m}\right\}
$$

where $\Phi^{\alpha}$ are the local independent constraint functions for the submanifold $M$. The rank of the $(\bar{m} \times(n+m))$-matrix

$$
\left(\frac{\partial \Phi^{\alpha}}{\partial x^{i}}, \frac{\partial \Phi^{\alpha}}{\partial y^{A}}\right)
$$

is maximun, that is, $\bar{m}$. On the other hand, since $\tau_{M}: M \rightarrow Q$ is a submersion, we deduce that there exists $v_{i} \in T_{e} M$ such that $\left(T \tau_{M}\right)\left(v_{i}\right)=\frac{\partial}{\partial x^{i}} \mid x$, for all $i \in$ $\{1, \ldots, m\}$. Thus,

which implies that

$$
v_{i}={\frac{\partial}{\partial x^{i}}}_{\mid e}+v_{i}^{A}{\frac{\partial}{\partial y^{A}}}_{\mid e}
$$

$$
{\frac{\partial \Phi^{\alpha}}{\partial x^{i}} \mid e}^{\mid}=-v_{i}^{A} \frac{\partial \Phi^{\alpha}}{\partial y^{A}}{ }_{\mid e} \text {, for } \alpha \in\{1, \ldots, \bar{m}\} \text { and } i \in\{1, \ldots, m\} \text {. }
$$

Therefore, the rank of the matrix

$$
\left(\frac{\partial \Phi^{\alpha}}{\partial y^{A}}\right)_{\alpha=1, \ldots, \bar{m} ; A=1, \ldots, n}
$$


is $\bar{m}$. We will suppose, without the loss of generality, that the $(\bar{m} \times \bar{m})$-matrix

$$
\left(\frac{\partial \Phi^{\alpha}}{\partial y^{B}}\right)_{\mid e}{ }_{\alpha=1, \ldots, \bar{m} ; B=1, \ldots, \bar{m}}
$$

is regular. Then, we will use the following notation

$$
y^{A}=\left(y^{\alpha}, y^{a}\right),
$$

for $1 \leq A \leq n, 1 \leq \alpha \leq \bar{m}$ and $\bar{m}+1 \leq a \leq n$.

Now, using the implicit function theorem, we obtain that there exist an open subset $\widetilde{V}$ of $\tau^{-1}(U)$, an open subset $W \subseteq \mathbb{R}^{m+n-\bar{m}}$ and smooth real functions

$$
\Psi^{\alpha}: W \rightarrow \mathbb{R}, \quad \alpha=1, \ldots, \bar{m},
$$

such that

$$
M \cap \widetilde{V} \equiv\left\{\left(x^{i}, y^{A}\right) \in \widetilde{V} \mid y^{\alpha}=\Psi^{\alpha}\left(x^{i}, y^{a}\right), \alpha=1, \ldots, \bar{m}\right\} .
$$

Consequently, $\left(x^{i}, y^{a}\right)$ are local coordinates on $M$. We will denote by $\tilde{L}$ the restriction of $L$ to $M$.

Now, we will develop a geometric description of vakonomic mechanics on Lie algebroids, naturally generalizing the previous results of the third author and collaborators [9. Moreover, for the case $M=E$ we also generalize the formulation given by Skinner and Rusk [32, 33] for singular lagrangians to general Lie algebroids.

Consider the Whitney sum of $E^{*}$ and $E, E^{*} \oplus E$, and the canonical projections $p r_{1}: E^{*} \oplus E \longrightarrow E^{*}$ and $p r_{2}: E^{*} \oplus E \longrightarrow E$. Now, let $W_{0}$ be the submanifold $W_{0}=p r_{2}^{-1}(M)=E^{*} \times_{Q} M$ and the restrictions $\pi_{1}=p r_{1 \mid W_{0}}$ and $\pi_{2}=p r_{2 \mid W_{0}}$. Also denote by $\nu: W_{0} \longrightarrow Q$ the canonical projection. The following diagrams illustrate the situation
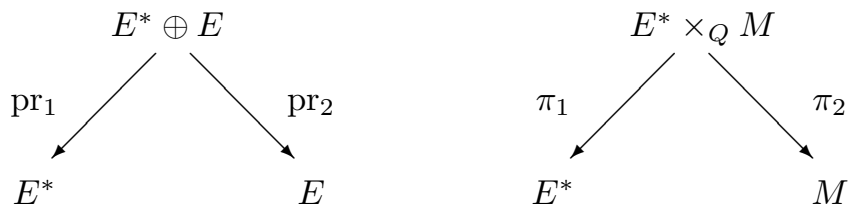

Next, we consider the prolongation of the Lie algebroid $E$ over $\tau^{*}: E^{*} \rightarrow Q$ (respectively, $\nu: W_{0} \rightarrow Q$ ). We will denote this Lie algebroid by $\mathcal{T}^{E} E^{*}$ (respectively, $\left.\mathcal{T}^{E} W_{0}\right)$. Moreover, we can prolong $\pi_{1}: W_{0} \rightarrow E^{*}$ to a morphism of Lie algebroids $\mathcal{T} \pi_{1}: \mathcal{T}^{E} W_{0} \rightarrow \mathcal{T}^{E} E^{*}$ defined by $\mathcal{T} \pi_{1}=\left(I d, T \pi_{1}\right)$.

If $\left(x^{i}, p_{A}\right)$ are the local coordinates on $E^{*}$ associated with the local basis $\left\{e_{A}\right\}$ of $\Gamma(E)$, then $\left(x^{i}, p_{A}, y^{a}\right)$ are local coordinates for $W_{0}$ and we may consider the local basis $\left\{y_{A}, \mathcal{P}^{A}, \mathcal{V}_{a}\right\}$ of $\Gamma\left(\mathcal{T}^{E} W_{0}\right)$ defined by

$$
\begin{aligned}
y_{A}\left(e^{*}, \check{e}\right) & =\left(e_{A}(x), \rho_{A}^{i} \frac{\partial}{\partial x^{i}} \mid e^{*}\right. \\
\mathcal{P}^{A}\left(e^{*}, \check{e}\right) & =\left(0,{\frac{\partial}{\partial p_{A}}}_{\mid e^{*}}, 0\right), \\
\mathcal{V}_{a}\left(e^{*}, \check{e}\right) & =\left(0,0,{\frac{\partial}{\partial y^{a}}}_{\mid \check{e}}\right),
\end{aligned}
$$

where $\left(e^{*}, \check{e}\right) \in W_{0}$ and $\nu\left(e^{*}, \check{e}\right)=x$. If $\left(\llbracket \cdot, \cdot \rrbracket^{\nu}, \rho^{\nu}\right)$ is the Lie algebroid structure on $\mathcal{T}^{E} W_{0}$, we have that

$$
\llbracket y_{A}, y_{B} \rrbracket^{\nu}=\mathcal{C}_{A B}^{C} y_{C},
$$

and the rest of the fundamental Lie brackets are zero. Moreover,

$$
\rho^{\nu}\left(y_{A}\right)=\rho_{A}^{i} \frac{\partial}{\partial x^{i}}, \quad \rho^{\nu}\left(\mathcal{P}^{A}\right)=\frac{\partial}{\partial p_{A}}, \quad \rho^{\nu}\left(\mathcal{V}_{a}\right)=\frac{\partial}{\partial y^{a}} .
$$


The Pontryagin Hamiltonian $H_{W_{0}}$ is a function in $W_{0}=E^{*} \times_{Q} M$ given by

$$
H_{W_{0}}\left(e^{*}, \check{e}\right)=\left\langle e^{*}, \check{e}\right\rangle-\tilde{L}(\check{e}),
$$

or, in local coordinates,

$$
H_{W_{0}}\left(x^{i}, p_{A}, y^{a}\right)=p_{a} y^{a}+p_{\alpha} \Psi^{\alpha}\left(x^{i}, y^{a}\right)-\tilde{L}\left(x^{i}, y^{a}\right) .
$$

Moreover, one can consider the presymplectic 2-section $\Omega_{0}=\left(\mathcal{T} \pi_{1}, \pi_{1}\right)^{*} \Omega_{E}$, where $\Omega_{E}$ is the canonical symplectic section on $\mathcal{T}^{E} E^{*}$ defined in Equation (2.5). In local coordinates,

$$
\Omega_{0}=y^{A} \wedge \mathcal{P}_{A}+\frac{1}{2} \varrho_{A B}^{C} p_{C} y^{A} \wedge y^{B}
$$

Therefore, we have the triple $\left(\mathcal{T}^{E} W_{0}, \Omega_{0}, d^{\mathcal{T}^{E}} W_{0} H_{W_{0}}\right)$ as a presymplectic hamiltonian system.

Definition 5.1. The vakonomic problem on Lie algebroids is find the solutions for the equation

$$
i_{X} \Omega_{0}=d^{\mathfrak{T}^{E}} W_{0} H_{W_{0}},
$$

that is, to solve the constraint algorithm for $\left(\mathcal{T}^{E} W_{0}, \Omega_{0}, d^{\mathcal{T}^{E}} W_{0} H_{W_{0}}\right)$.

In local coordinates, we have that

$$
d^{\mathcal{T}^{E} W_{0}} H_{W_{0}}=\left(p_{\alpha} \frac{\partial \Psi^{\alpha}}{\partial x^{i}}-\frac{\partial \tilde{L}}{\partial x^{i}}\right) \rho_{A}^{i} y^{A}+\Psi^{\alpha} \mathcal{P}_{\alpha}+y^{a} \mathcal{P}_{a}+\left(p_{a}+p_{\alpha} \frac{\partial \Psi^{\alpha}}{\partial y^{a}}-\frac{\partial \tilde{L}}{\partial y^{a}}\right) \mathcal{V}^{a}
$$

If we apply the constraint algorithm,

$$
W_{1}=\left\{w \in E^{*} \times_{Q} M \mid d^{\mathfrak{T}^{E} W_{0}} H_{W_{0}}(w)(Y)=0, \quad \forall Y \in \operatorname{Ker} \Omega_{0}(w)\right\} .
$$

Since $\operatorname{Ker} \Omega_{0}=\operatorname{span}\left\{\mathcal{V}_{a}\right\}$, we get that $W_{1}$ is locally characterized by the equations

$$
\varphi_{a}=d^{\mathcal{T}^{E}} W_{0} H_{W_{0}}\left(\mathcal{V}_{a}\right)=p_{a}+p_{\alpha} \frac{\partial \Psi^{\alpha}}{\partial y^{a}}-\frac{\partial \tilde{L}}{\partial y^{a}}=0,
$$

or

$$
p_{a}=\frac{\partial \tilde{L}}{\partial y^{a}}-p_{\alpha} \frac{\partial \Psi^{\alpha}}{\partial y^{a}}, \quad \bar{m}+1 \leq a \leq n .
$$

Let us also look for the expression of $X$ satisfying Eq. (5.3). A direct computation shows that

$$
X=y^{a} y_{a}+\Psi^{\alpha} y_{\alpha}+\left[\left(\frac{\partial \tilde{L}}{\partial x^{i}}-p_{\alpha} \frac{\partial \Psi^{\alpha}}{\partial x^{i}}\right) \rho_{A}^{i}-y^{a} \mathcal{C}_{A a}^{B} p_{B}-\Psi^{\alpha} \mathcal{C}_{A \alpha}^{B} p_{B}\right] \mathcal{P}^{A}+\Upsilon^{a} \mathcal{V}_{a}
$$

Therefore, the vakonomic equations are

$$
\left\{\begin{array}{l}
\dot{x}^{i}=y^{a} \rho_{a}^{i}+\Psi^{\alpha} \rho_{\alpha}^{i} \\
\dot{p}_{\alpha}=\left(\frac{\partial \tilde{L}}{\partial x^{i}}-p_{\beta} \frac{\partial \Psi^{\beta}}{\partial x^{i}}\right) \rho_{\alpha}^{i}-y^{a} \mathcal{C}_{\alpha a}^{B} p_{B}-\Psi^{\beta} \mathcal{C}_{\alpha \beta}^{B} p_{B} \\
\frac{d}{d t}\left(\frac{\partial \tilde{L}}{\partial y^{a}}-p_{\alpha} \frac{\partial \Psi^{\alpha}}{\partial y^{a}}\right)=\left(\frac{\partial \tilde{L}}{\partial x^{i}}-p_{\alpha} \frac{\partial \Psi^{\alpha}}{\partial x^{i}}\right) \rho_{a}^{i}-y^{b} \mathcal{C}_{a b}^{B} p_{B}-\Psi^{\alpha} \mathcal{C}_{a \alpha}^{B} p_{B}
\end{array}\right.
$$

Remark 5.2. We note that the vakonomic equations can be obtained following a constrained variational principle. Below, we will show this variational way of obtaining these equations (see Subsection 5.2). 
Of course, we know that there exist sections $X$ of $\mathcal{T}^{E} W_{0}$ along $W_{1}$ satisfying (5.3), but they may not be sections of $\left(\rho^{\nu}\right)^{-1}\left(T W_{1}\right)=\mathcal{T}^{E} W_{1}$, in general. Then, following the procedure detailed in Section 3 , we obtain a sequence of embedded submanifolds

$$
\ldots \hookrightarrow W_{k+1} \hookrightarrow W_{k} \hookrightarrow \ldots \hookrightarrow W_{2} \hookrightarrow W_{1} \hookrightarrow W_{0}=E^{*} \times_{Q} M .
$$

If the algorithm stabilizes, then we find a final constraint submanifold $W_{f}$ on which at least a section $X \in \Gamma\left(\mathcal{T}^{E} W_{f}\right)$ verifies

$$
\left(i_{X} \Omega_{0}=d^{\mathcal{T}^{E} W_{0}} H_{W_{0}}\right)_{\mid W_{f}} .
$$

As in [9], we analyze the case when $W_{f}=W_{1}$. Consider the restriction $\Omega_{1}$ of $\Omega_{0}$ to $\mathcal{T}^{E} W_{1}$

Proposition 5.3. $\Omega_{1}$ is a symplectic section of the Lie algebroid $\mathcal{T}^{E} W_{1}$ if and only if for any system of coordinates $\left(x^{i}, p_{A}, y^{a}\right)$ on $W_{0}$ we have that

$$
\operatorname{det}\left(\frac{\partial^{2} \tilde{L}}{\partial y^{a} \partial y^{b}}-p_{\alpha} \frac{\partial^{2} \Psi^{\alpha}}{\partial y^{a} \partial y^{b}}\right) \neq 0, \text { for all point in } W_{1} \text {. }
$$

Proof. It is clear that $d^{\mathcal{T}^{E}} W_{1} \Omega_{1}=0$.

On the other hand, if $w \in W_{1}$ then, since the elements $\left(d^{\mathfrak{T}^{E}} W_{0} \varphi_{a}\right)(w)$ are independent in $\left(\mathcal{T}_{w}^{E} W_{0}\right)^{*}$, we have that

$$
\left(\mathcal{T}_{w}^{E} W_{1}\right)^{\circ}=<\left(d^{\mathcal{T}^{E}} W_{0} \varphi_{a}\right)(w)>
$$

(note that $\left.\operatorname{dim}\left(\mathcal{T}_{w}^{E} W_{1}\right)^{\circ}=n-\bar{m}\right)$. Moreover, using a well-known result (see, for instance, 24]), we deduce that

$$
\operatorname{dim}\left(\mathcal{T}_{w}^{E} W_{1}\right)^{\perp, \Omega_{0}}=\operatorname{dim}\left(\mathcal{T}_{w}^{E} W_{0}\right)-\operatorname{dim}\left(\mathcal{T}_{w}^{E} W_{1}\right)+\operatorname{dim}\left(\operatorname{Ker} \Omega_{0}(w) \cap \mathcal{T}_{w}^{E} W_{1}\right)
$$

Now, suppose that $\Omega_{1}$ is a nondegenerate 2 -section on $\mathcal{T}_{w}^{E} W_{1} \rightarrow W_{1}$. Then, it is clear that

$$
\operatorname{Ker} \Omega_{0}(w) \cap \mathcal{T}_{w}^{E} W_{1}=\{0\} .
$$

Thus, the matrix $\left(d^{\mathcal{T}^{E}} W_{0} \varphi_{a}\right)(w)\left(\mathcal{V}_{b}(w)\right)$ is regular, that is,

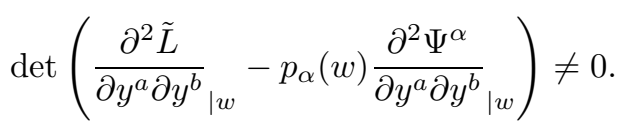

Conversely, assume that the matrix $\left(d^{\mathcal{T}^{E}} W_{0} \varphi_{a}\right)(w)\left(\mathcal{V}_{b}(w)\right)$ is regular, then

$$
\operatorname{Ker} \Omega_{0}(w) \cap \mathcal{T}_{w}^{E} W_{1}=\{0\} .
$$

This implies that (see (5.4)

$$
\operatorname{dim}\left(\mathcal{T}_{w}^{E} W_{1}\right)^{\perp, \Omega_{0}}=\operatorname{dim}\left(\mathcal{T}_{w}^{E} W_{0}\right)-\operatorname{dim}\left(\mathcal{T}_{w}^{E} W_{1}\right)=\operatorname{dim}\left(\operatorname{Ker} \Omega_{0}(w)\right) .
$$

Therefore, since $\operatorname{Ker} \Omega_{0}(w) \subseteq\left(\mathcal{T}_{w}^{E} W_{1}\right)^{\perp, \Omega_{0}}$, it follows that

$$
\operatorname{Ker} \Omega_{0}(w)=\left(\mathcal{T}_{w}^{E} W_{1}\right)^{\perp, \Omega_{0}},
$$

and, consequently,

$$
\left(\mathcal{T}_{w}^{E} W_{1}\right) \cap\left(\mathcal{T}_{w}^{E} W_{1}\right)^{\perp, \Omega_{0}}=\{0\},
$$

that is, $\Omega_{1}(w)$ is a nondegenerate 2 -section.

In what follows, we will use the following notation

$$
\mathcal{R}_{a b}=\frac{\partial \tilde{L}}{\partial y^{a} \partial y^{b}}-p_{\alpha} \frac{\partial^{2} \Psi^{\alpha}}{\partial y^{a} \partial y^{b}}, \text { for all } a \text { and } b .
$$


Remark 5.4. Suppose that the submanifold $M$ is a real vector subbundle $D$ (over Q) of $E$ (note that $\tau_{D}=\tau_{\mid D}: D \rightarrow M$ is a surjective submersion). Then, we may consider local coordinates $\left(x^{i}\right)$ on an open subset $U$ of $Q$ and a local basis $\left\{e_{\alpha}, e_{a}\right\}$ of $\Gamma(E)$ on $U$ such that $\left\{e_{a}\right\}$ is a local basis of $\Gamma(D)$. Thus, if $\left(x^{i}, y^{\alpha}, y^{a}\right)$ are the corresponding local coordinates on $\tau^{-1}(U)$, we have that

$$
\tau_{D}^{-1}(U)=D \cap \tau^{-1}(U) \equiv\left\{\left(x^{i}, y^{\alpha}, y^{a}\right) \in \tau^{-1}(U) / y^{\alpha}=0\right\} .
$$

In other words, the local function $\Psi^{\alpha}$ is the zero function, for all $\alpha$. Therefore, in this case,

$$
\mathcal{R}_{a b}=\frac{\partial^{2} \tilde{L}}{\partial y^{a} \partial y^{b}}, \text { for all } a \text { and } b .
$$

We remark that the condition

$$
\operatorname{det}\left(\frac{\partial^{2} \tilde{L}}{\partial y^{a} \partial y^{b}}\right) \neq 0
$$

implies that the corresponding nonholonomic problem determined by the pair $(L, D)$ has a unique solution (see [8]).

Remark 5.5. We remark that the condition $\operatorname{det}\left(\mathcal{R}_{a b}\right) \neq 0$ implies that the matrix $\left(\frac{\partial \varphi_{a}}{\partial y^{b}}\right)_{a, b=\bar{m}+1, \ldots, n}$ is regular. Thus, using the implicit theorem function, we deduce that $\left(x^{i}, p_{A}, y^{a}\right)$ are local coordinates for $W_{0}$ on an open subset $A_{0} \subseteq W_{0}$ in such a way that there exist an open subset $\tilde{W} \subseteq \mathbb{R}^{m+n}$ and smooth real functions

$$
\mu^{a}: \tilde{W} \rightarrow \mathbb{R}, \quad a=\bar{m}+1, \ldots, n,
$$

such that

$$
W_{1} \cap A_{0}=\left\{\left(x^{i}, p_{A}, y^{a}\right) \in A_{0} / y^{a}=\mu^{a}\left(x^{i}, p_{A}\right), a=\bar{m}+1, \ldots, n\right\} .
$$

Therefore, a local basis of $\Gamma\left(\mathcal{T}^{E} W_{1}\right)$ is given by

$$
\left\{y_{A 1}=\left(y_{A}+\rho_{A}^{i} \frac{\partial \mu^{a}}{\partial x^{i}} \mathcal{V}_{a}\right)_{\mid W_{1}}, \mathcal{P}_{1}^{A}=\left(\mathcal{P}^{A}+\frac{\partial \mu^{a}}{\partial p_{A}} \mathcal{V}_{a}\right)_{\mid W_{1}}\right\}
$$

This implies that

$$
\left\{y_{A 1}, \mathcal{P}_{1}^{A},\left(\mathcal{V}_{a}\right)_{\mid W_{1}}\right\}
$$

is a local basis of $\Gamma\left(\mathcal{T}_{W_{1}}^{E} W_{0}\right)$. Note that, from (5.5), it follows that $\left(x^{i}, p_{A}\right)$ are local coordinates on $W_{1}$. Moreover, if $\nu_{1}: W_{1} \rightarrow Q$ is the canonical projection and $\left(\llbracket \cdot, \cdot \rrbracket^{\nu_{1}}, \rho^{\nu_{1}}\right)$ is the Lie algebroid structure on $\mathcal{T}^{E} W_{1} \rightarrow W_{1}$, we have that

$$
\llbracket y_{A 1}, y_{B 1} \rrbracket^{\nu_{1}}=C_{A B}^{C} y_{C 1}
$$

and the rest of the fundamental Lie brackets are zero. In addition,

$$
\rho^{\nu_{1}}\left(y_{A 1}\right)=\rho_{A}^{i} \frac{\partial}{\partial x^{i}}, \quad \rho^{\nu_{1}}\left(\mathcal{P}_{1}^{A}\right)=\frac{\partial}{\partial p_{A}} \text {. }
$$

Now, we will prove the following result.

Theorem 5.6. If $\Omega_{1}$ is a symplectic 2 -section on the Lie algebroid $\mathcal{T}^{E} W_{1} \rightarrow W_{1}$ then there exists a unique section $\xi_{1}$ of $\mathcal{T}^{E} W_{1} \rightarrow W_{1}$ whose integral curves are solutions of the vakonomic equations for the system $(L, M)$. In fact, if $H_{W_{1}}$ is the restriction to $W_{1}$ of the Pontryagin Hamiltonian $H_{W_{0}}$, then $\xi_{1}$ is the Hamiltonian section of $H_{W_{1}}$ with respect to the symplectic section $\Omega_{1}$, that is,

$$
i_{\xi_{1}} \Omega_{1}=d^{\mathcal{T}^{E} W_{1}} H_{W_{1}}
$$


Proof. We have that (see (5.1)

$$
\left(d^{\mathcal{T}^{E}} W_{0} H_{W_{0}}\right)\left(\mathcal{V}_{a}\right)=\rho^{\nu}\left(\mathcal{V}_{a}\right)\left(H_{W_{0}}\right)=\left(\frac{\partial H_{W_{0}}}{\partial y^{a}}\right)=\varphi_{a} .
$$

Therefore, from (5.2), it follows that

$$
\left(\Omega_{0}\right)_{\mid W_{1}}\left(\xi_{1},\left(\mathcal{V}_{a}\right)_{\mid W_{1}}\right)=\left(d^{\mathcal{T}^{E} W_{0}} H_{W_{0}}\right)\left(\mathcal{V}_{a}\right)_{\mid W_{1}}=0 .
$$

This implies that

$$
i_{\xi_{1}}\left(\Omega_{0}\right)_{\mid W_{1}}=\left(d^{\mathcal{T}^{E}} W_{0} H_{W_{0}}\right)_{\mid W_{1}}
$$

and, consequently, the integral curves of $\xi_{1}$ are solutions of the vakonomic equations for the system $(L, M)$.

Moreover, if $\xi_{1}^{\prime} \in \Gamma\left(\mathcal{T}^{E} W_{1}\right)$ is another solution of the equation

$$
i_{\xi_{1}^{\prime}}\left(\Omega_{0}\right)_{\mid W_{1}}=\left(d^{\mathcal{T}^{E}} W_{0} H_{W_{0}}\right)_{\mid W_{1}}
$$

then it is also a solution of the equation

$$
i_{\xi_{1}^{\prime}} \Omega_{1}=d^{\mathcal{T}^{E} W_{1}} H_{W_{1}}
$$

which implies that $\xi_{1}^{\prime}=\xi_{1}$.

Suppose that $\left(x^{i}, p_{A}\right)$ are local coordinates on $W_{1}$ as in Remark 5.5] and that $\left\{y_{A 1}, \mathcal{P}_{1}^{A}\right\}$ is the corresponding local basis of $\Gamma\left(\mathcal{T}^{E} W_{1}\right)$. Then, if $\left\{y_{1}^{A}, \mathcal{P}_{A 1}\right\}$ is the dual basis of $\left\{y_{A 1}, \mathcal{P}_{1}^{A}\right\}$, we have that (see (5.2)

$$
\Omega_{1}=y_{1}^{A} \wedge \mathcal{P}_{A 1}+\frac{1}{2} \mathcal{C}_{A B}^{C} p_{C} y_{1}^{A} \wedge y_{1}^{B} .
$$

On the other hand, from (5.6), it follows that

$$
d^{\mathcal{T}^{E} W_{1}} H_{W_{1}}=\rho_{A}^{i} \frac{\partial H_{W_{1}}}{\partial x^{i}} y_{1}^{A}+\frac{\partial H_{W_{1}}}{\partial p_{A}} \mathcal{P}_{A 1}
$$

Therefore,

Note that

$$
\xi_{1}=\frac{\partial H_{W_{1}}}{\partial p_{A}} y_{A 1}-\left(\mathcal{C}_{A B}^{C} p_{C} \frac{\partial H_{W_{1}}}{\partial p_{B}}+\rho_{A}^{i} \frac{\partial H_{W_{1}}}{\partial x^{i}}\right) \mathcal{P}_{1}^{A}
$$

$\frac{\partial H_{W_{1}}}{\partial x^{i}}=\left(\left(\frac{\partial}{\partial x^{i}}+\frac{\partial \mu^{a}}{\partial x^{i}} \frac{\partial}{\partial y^{a}}\right)\left(H_{W_{0}}\right)\right)_{\mid W_{1}}, \frac{\partial H_{W_{1}}}{\partial p_{A}}=\left(\left(\frac{\partial}{\partial p_{A}}+\frac{\partial \mu^{a}}{\partial p_{A}} \frac{\partial}{\partial y^{a}}\right)\left(H_{W_{0}}\right)\right)_{\mid W_{1}}$

which implies that

$$
\begin{aligned}
\xi_{1}\left(x^{j}, p_{B}\right)= & \mu^{a}\left(x^{j}, p_{B}\right) y_{a 1}+\Psi^{\alpha}\left(x^{j}, \mu^{a}\left(x^{j}, p_{B}\right)\right) y_{\alpha 1} \\
& -\left(\mathcal{C}_{A a}^{b} p_{b} \mu^{a}\left(x^{j}, p_{B}\right)+\bigodot_{A \alpha}^{b} p_{b} \Psi^{\alpha}\left(x^{j}, \mu^{a}\left(x^{j}, p_{B}\right)\right)\right. \\
& \left.+\rho_{A}^{i}\left(p_{\alpha}{\frac{\partial \Psi^{\alpha}}{\partial x^{i} \mid\left(x^{j}, \mu^{a}\left(x^{j}, p_{B}\right)\right)}}-\frac{\partial \tilde{L}}{\partial x^{i} \mid\left(x^{j}, \mu^{a}\left(x^{j}, p_{B}\right)\right)}\right)\right) \mathcal{P}_{1}^{A} .
\end{aligned}
$$

Now, we will introduce the following definition.

Definition 5.7. The vakonomic system $(L, M)$ on the Lie algebroid $\tau: E \rightarrow Q$ is said to be regular if $\Omega_{1}$ is a symplectic 2 -section of the Lie algebroid $\mathcal{T}^{E} W_{1} \rightarrow W_{1}$.

Suppose that $(L, M)$ is a regular vakonomic system and that $F_{1} \in C^{\infty}\left(W_{1}\right)$. Then, the Hamiltonian section of $F_{1}$ with respect to $\Omega_{1}$ is the section $\mathcal{H}_{F_{1}}^{\Omega_{1}}$ of the Lie algebroid $\mathcal{T}^{E} W_{1} \rightarrow W_{1}$ which is characterized by the equation

$$
i\left(\mathcal{H}_{F 1}^{\Omega_{1}}\right) \Omega_{1}=d^{\mathcal{T}^{E} W_{1}} F_{1} .
$$

Note that $\xi_{1}=\mathcal{H}_{H_{W_{1}}}^{\Omega_{1}}$. 
Using the Hamiltonian sections, one may introduce a bracket of functions $\{\cdot, \cdot\}_{(L, M)}$ : $C^{\infty}\left(W_{1}\right) \times C^{\infty}\left(W_{1}\right) \rightarrow C^{\infty}\left(W_{1}\right)$ as follows

$$
\left\{F_{1}, G_{1}\right\}_{(L, M)}=\Omega_{1}\left(\mathcal{H}_{F_{1}}^{\Omega_{1}}, \mathcal{H}_{G_{1}}^{\Omega_{1}}\right)=\rho^{\nu_{1}}\left(\mathcal{H}_{G_{1}}^{\Omega_{1}}\right)\left(F_{1}\right)
$$

for $F_{1}, G_{1} \in C^{\infty}\left(W_{1}\right)$. The bracket $\{\cdot, \cdot\}_{(L, M)}$ is called the vakonomic bracket associated with the system $(L, M)$.

Theorem 5.8. The vakonomic bracket $\{\cdot, \cdot\}_{(L, M)}$ associated with a regular vakonomic system is a Poisson bracket on $W_{1}$. Moreover, if $F_{1} \in C^{\infty}\left(W_{1}\right)$ then the temporal evolution of $F_{1}, \dot{F}_{1}$, is given by

$$
\dot{F}_{1}=\left\{F_{1}, H_{W_{1}}\right\}_{(L, M)} \text {. }
$$

Proof. Let $b_{\Omega_{1}}: \mathcal{T}^{E} W_{1} \rightarrow\left(\mathcal{T}^{E} W_{1}\right)^{*}$ be the musical isomorphism induced by $\Omega_{1}$ which is defined by

$$
b_{\Omega_{1}}\left(X_{1}\right)=i\left(X_{1}\right) \Omega_{1}\left(w_{1}\right), \quad \text { for } X_{1} \in \mathcal{T}_{w_{1}}^{E} W_{1} \text { and } w_{1} \in W_{1}
$$

Then, we may introduce the section $\Lambda_{1}$ of the vector bundle $\Lambda^{2} \mathcal{T}^{E} W_{1} \rightarrow W_{1}$ as follows

$$
\Lambda_{1}\left(\alpha_{1}, \beta_{1}\right)=\Omega_{1}\left(b_{\Omega_{1}}^{-1} \circ \alpha_{1}, b_{\Omega_{1}}^{-1} \circ \beta_{1}\right), \quad \text { for } \alpha_{1}, \beta_{1} \in \Gamma\left(\mathcal{T}^{E} W_{1}\right)^{*} .
$$

If $\llbracket \cdot, \cdot \rrbracket^{\nu_{1}}$ is the Schouten-Nijenhuis bracket associated with the Lie algebroid $\mathcal{T}^{E} W_{1}$ $\rightarrow W_{1}$ then, using that $d^{\mathcal{T}^{E}} W_{1} \Omega_{1}=0$, one may prove that

$$
\llbracket \Lambda_{1}, \Lambda_{1} \rrbracket^{\nu_{1}}=0 .
$$

Thus, $\Lambda_{1}$ is a triangular matrix, the pair $\left(\mathcal{T}^{E} W_{1},\left(\mathcal{T}^{E} W_{1}\right)^{*}\right)$ is a triangular Lie bialgebroid in the sense of Mackenzie and $\mathrm{Xu}$ (see Section 4 in [26]) and $\{\cdot, \cdot\}_{(L, M)}$ is a Poisson bracket on $W_{1}$ (see Proposition 3.6 in [26]).

On the other hand, if $F_{1} \in C^{\infty}\left(W_{1}\right)$ and $\rho^{\nu_{1}}$ is the anchor map of the Lie algebroid $\mathcal{T}^{E} W_{1} \rightarrow W_{1}$ then

$$
\dot{F}_{1}=\rho^{\nu_{1}}\left(\xi_{1}\right)\left(F_{1}\right)=\rho^{\nu_{1}}\left(\mathcal{H}_{H_{W_{1}}}^{\Omega_{1}}\right)\left(F_{1}\right)=\left(d^{\mathcal{T}^{E} W_{1}} F_{1}\right)\left(\mathcal{H}_{H_{W_{1}}}^{\Omega_{1}}\right) .
$$

Therefore,

$$
\dot{F}_{1}=\left\{F_{1}, H_{W_{1}}\right\}_{(L, M)}
$$

Suppose that $\left(x^{i}, p_{A}\right)$ are local coordinates on $W_{1}$ as in Remark 5.5 and that $\left\{y_{A 1}, \mathcal{P}_{1}^{A}\right\}$ is the corresponding local basis of $\Gamma\left(\mathcal{T}^{E} W_{1}\right)$. If $F_{1} \in C^{\infty}\left(W_{1}\right)$ then, from (5.6), it follows that

$$
d^{\mathcal{T}^{E} W_{1}} F_{1}=\rho_{A}^{i} \frac{\partial F_{1}}{\partial x^{i}} y_{1}^{A}+\frac{\partial F_{1}}{\partial p_{A}} \mathcal{P}_{A 1}
$$

Consequently, using (5.7), we deduce that

$$
\mathcal{H}_{F_{1}}^{\Omega_{1}}=\frac{\partial F_{1}}{\partial p_{A}} y_{A 1}-\left(\mathcal{C}_{A B}^{C} p_{C} \frac{\partial F_{1}}{\partial p_{B}}+\rho_{A}^{i} \frac{\partial F_{1}}{\partial x^{i}}\right) \mathcal{P}_{1}^{A} .
$$

This implies that

$$
\left\{F_{1}, G_{1}\right\}_{(L, M)}=\rho_{A}^{i}\left(\frac{\partial F_{1}}{\partial x^{i}} \frac{\partial G_{1}}{\partial p_{A}}-\frac{\partial F_{1}}{\partial p_{A}} \frac{\partial G_{1}}{\partial x^{i}}\right)-\mathcal{C}_{A B}^{C} p_{C} \frac{\partial F_{1}}{\partial p_{A}} \frac{\partial G_{1}}{\partial p_{B}} .
$$

Now, we consider the linear Poisson structure

$$
\{\cdot, \cdot\}_{E^{*}}: C^{\infty}\left(E^{*}\right) \times C^{\infty}\left(E^{*}\right) \rightarrow C^{\infty}\left(E^{*}\right)
$$

on $E^{*}$ induced by the Lie algebroid structure on $E$ and the canonical symplectic structure $\Omega_{E}$ on $\mathcal{T}^{E} E^{*}$ (see Section 2 ). 
Corollary 5.9. If $(L, M)$ is a regular vakonomic system on a Lie algebroid $E$ then the restriction $\left(\pi_{1}\right)_{\mid W_{1}}: W_{1} \rightarrow E^{*}$ of $\pi_{1}: W_{0} \rightarrow E^{*}$ to $W_{1}$ is a local Poisson isomorphism. Moreover, if $\mathcal{T}\left(\pi_{1}\right)_{\mid W_{1}}: \mathcal{T}^{E} W_{1} \rightarrow \mathcal{T}^{E} E^{*}$ is the corresponding prolongation then the pair $\left(\mathcal{T}\left(\pi_{1}\right)_{\mid W_{1}},\left(\pi_{1}\right)_{\mid W_{1}}\right)$ is a local symplectomorphism between the symplectic Lie algebroids $\left(\mathcal{T}^{E} W_{1}, \Omega_{1}\right)$ and $\left(\mathcal{T}^{E} E^{*}, \Omega_{E}\right)$.

Proof. From Remark 5.5, we obtain that $\left(\pi_{1}\right)_{\mid W_{1}}: W_{1} \rightarrow E^{*}$ is a local diffeomorphism. Furthermore, using (2.2) and (5.8), we deduce that

$$
\left\{F \circ\left(\pi_{1}\right)_{\mid W_{1}}, G \circ\left(\pi_{1}\right)_{\mid W_{1}}\right\}_{(L, M)}=\{F, G\}_{E^{*}} \circ\left(\pi_{1}\right)_{\mid W_{1}},
$$

for $F, G \in C^{\infty}\left(E^{*}\right)$. This proves the first part of the corollary. The second part follows from the first one and using the fact that $\left(\mathcal{T}\left(\pi_{1}\right)_{\mid W_{1}},\left(\pi_{1}\right)_{\mid W_{1}}\right)^{*} \Omega_{E}=\Omega_{1}$.

Remark 5.10. If $\left(\pi_{1}\right)_{\mid W_{1}}: W_{1} \rightarrow E^{*}$ is a global diffeomorphism then we may consider the real function $H$ on $E^{*}$ defined by

$$
H=H_{W_{1}} \circ\left(\pi_{1}\right)_{\mid W_{1}}^{-1} .
$$

In addition, if $\mathcal{H}_{H}^{\Omega_{E}} \in \Gamma\left(\mathcal{T}^{E} E^{*}\right)$ is the Hamiltonian section of $H$ with respect to the symplectic section $\Omega_{E}$ then $\xi_{1}$ and $\mathcal{H}_{H}^{\Omega_{E}}$ are $\left(\mathcal{T}\left(\pi_{1}\right)_{\mid W_{1}},\left(\pi_{1}\right)_{\mid W_{1}}\right)$-related, that is,

$$
\mathcal{T}\left(\pi_{1}\right)_{\mid W_{1}} \circ \xi_{1}=\mathcal{H}_{H}^{\Omega_{E}} \circ\left(\pi_{1}\right)_{\mid W_{1}} .
$$

5.2. The variational point of view. As it is well known, the dynamics in a vakonomic system is obtained through the application of a constrained variational principle [1. More precisely, let $Q$ be the configuration manifold, $L: T Q \rightarrow \mathbb{R}$ be the Lagrangian function and $M \subset T Q$ be the constraint submanifold. Then, one can consider the set of twice differentiable curves which connect two arbitrary points $x, y \in Q$ as

$$
\mathcal{C}^{2}(x, y)=\left\{a:\left[t_{0}, t_{1}\right] \rightarrow Q \mid a \text { is } C^{2}, a\left(t_{0}\right)=x \text { and } a\left(t_{1}\right)=y\right\} .
$$

This is a smooth manifold of infinite dimension and, given $a \in \mathcal{C}^{2}(x, y)$, the tangent space of $\mathfrak{C}^{2}(x, y)$ is described as

$T_{a} \mathrm{e}^{2}(x, y)=\left\{X:\left[t_{0}, t_{1}\right] \rightarrow T Q \mid X\right.$ is $C^{1}, X(t) \in T_{a(t)} Q, X\left(t_{0}\right)=0$ and $\left.X\left(t_{1}\right)=0\right\}$.

The elements of $T_{a} \mathrm{e}^{2}(x, y)$ can be seen as the infinitesimal variations of the curve $a$. Next, one can introduce the action functional $\delta S: \mathcal{C}^{2}(x, y) \rightarrow \mathbb{R}$ defined by $a \mapsto \delta S(a)=\int_{t_{0}}^{t_{1}} L(\dot{a}(t)) d t$. Thus, the associated vakonomic problem $(Q, L, M)$ consists of extremizing the functional $\delta S$ restricted to the subset $\widetilde{\mathfrak{C}}^{2}(x, y)$, which is given by

$$
\widetilde{\mathfrak{C}}^{2}(x, y)=\left\{a \in \mathcal{C}^{2}(x, y) \mid \dot{a}(t) \in M_{a(t)}=M \cap \tau_{Q}^{-1}(a(t)), \forall t \in\left[t_{0}, t_{1}\right]\right\},
$$

that is, $a \in \widetilde{\mathcal{C}}^{2}(x, y)$ is a solution of the vakonomic system if $a$ is a critical point of $\delta S_{\mid \widetilde{\mathfrak{C}}^{2}(x, y)}$. In the particular case when we do not have constraints, i.e. $M=T Q$, then we recover the variational way to obtain Euler-Lagrange equations.

Now, consider a Lie algebroid $\tau: E \rightarrow Q$ and $L: E \rightarrow \mathbb{R}$ a Lagrangian function on it. In 30 it is shown how to obtain the Euler-Lagrange equations (on the Lie algebroid) from a variational point of view. Let us recall some aspects related with this formulation. First, the set of $E$-paths is defined by

$$
\mathcal{A} d m\left(\left[t_{0}, t_{1}\right], E\right)=\left\{a:\left[t_{0}, t_{1}\right] \rightarrow E \mid \rho \circ a=\frac{d}{d t}(\tau \circ a)\right\} .
$$


This set is a Banach submanifold of the set of $C^{1}$ paths in $E$ whose base path is $C^{2}$. Two $E$-paths $a_{0}$ and $a_{1}$ are said to be $E$-homotopic if there exists a morphism of Lie algebroids

$$
\Phi: T I \times T J \rightarrow E,
$$

where $I=[0,1], J=\left[t_{0}, t_{1}\right], a(s, t)=\Phi\left(\left.\partial_{t}\right|_{(s, t)}\right)$ and $b(s, t)=\Phi\left(\left.\partial_{s}\right|_{(s, t)}\right)$, such that

$$
\begin{array}{llrl}
a(0, t) & =a_{0}(t), & b\left(s, t_{0}\right) & =0, \\
a(1, t) & =a_{1}(t), & b\left(s, t_{1}\right) & =0 .
\end{array}
$$

$E$-homotopy classes induce a second differentiable Banach manifold structure on $\mathcal{A} d m\left(\left[t_{0}, t_{1}\right], E\right)$. The set of $E$-admissible paths with this second manifold structure will be denoted by $\mathcal{P}\left(\left[t_{0}, t_{1}\right], E\right)$. In addition, at each $E$-admissible curve $a$, the tangent space is given in terms of the so-called complete lifts of sections. In fact,

$$
T_{a} \mathcal{P}\left(\left[t_{0}, t_{1}\right], E\right)=\left\{\eta^{\mathbf{c}} \in T_{a} \mathcal{A} d m\left(\left[t_{0}, t_{1}\right], E\right) \mid \eta\left(t_{0}\right)=0 \quad \text { and } \quad \eta\left(t_{1}\right)=0\right\} .
$$

(for more details, see [5, 30]). We recall that if $\left\{e_{A}\right\}$ is a local basis for $E$ and $\eta$ is a time-dependent section locally given by

$$
\eta=\eta^{A} e_{A}
$$

then $\eta^{\mathbf{c}}$, the complete lift of $\eta$, is the vector field on $E$ given by

$$
\eta^{\mathbf{c}}=\eta^{A} \rho_{A}^{i} \frac{\partial}{\partial x^{i}}+\left(\rho_{B}^{i} \frac{\partial \eta^{C}}{\partial x^{i}}-\eta^{A} \mathcal{C}_{A B}^{C}\right) y^{B} \frac{\partial}{\partial y^{C}} .
$$

With the second manifold structure that it is introduced on the space of $E$-paths, it is possible to formulate the variational principle in a standard way. Let us fix two points $x, y \in M$ and consider the set $\mathcal{P}\left(\left[t_{0}, t_{1}\right], E\right)_{x}^{y}$ of $E$-paths with fixed base endpoints equal to $x$ and $y$, that is,

$$
\mathcal{P}\left(\left[t_{0}, t_{1}\right], E\right)_{x}^{y}=\left\{a \in \mathcal{P}\left(\left[t_{0}, t_{1}\right], E\right) \mid \tau\left(a\left(t_{0}\right)\right)=x \quad \text { and } \quad \tau\left(a\left(t_{1}\right)\right)=y\right\} .
$$

In this setting, for the action functional $\delta S: \mathcal{P}\left(\left[t_{0}, t_{1}\right], E\right) \rightarrow \mathbb{R}$ given by

$$
\delta S(a)=\int_{t_{0}}^{t_{1}} L(a(t)) d t
$$

the critical points of $\delta S$ on $\mathcal{P}\left(\left[t_{0}, t_{1}\right], E\right)_{x}^{y}$ are the curves $a \in \mathcal{P}\left(\left[t_{0}, t_{1}\right], E\right)_{x}^{y}$ which satisfy Euler-Lagrange equations (2.9) (see [30]).

Now, let $(L, M)$ be a vakonomic system on the Lie algebroid $\tau: E \rightarrow Q$. We will denote by $\mathcal{P}\left(\left[t_{0}, t_{1}\right], M\right)_{x}^{y}$ the set of $E$-paths in $M$ with fixed base endpoints equal to $x$ and $y$

$$
\mathcal{P}\left(\left[t_{0}, t_{1}\right], M\right)_{x}^{y}=\left\{a \in \mathcal{P}\left(\left[t_{0}, t_{1}\right], E\right)_{x}^{y} \mid a(t) \in M, \forall t \in\left[t_{0}, t_{1}\right]\right\} .
$$

We are going to consider infinitesimal variations (that is, complete lifts $\eta^{\mathbf{c}}$ ) tangent to the constraint submanifold $M$. We are going to assume that there exist enough infinitesimal variations (that is, we are studying the so-called normal solutions of the vakonomic problem). Since $M$ is locally given by $y^{\alpha}-\Psi^{\alpha}\left(x^{i}, y^{a}\right)=0$, we deduce that the allowed infinitesimal variations must satisfy

$$
\eta^{\mathbf{c}}\left(y^{\alpha}-\Psi^{\alpha}\left(x^{i}, y^{a}\right)\right)=0, \quad \eta\left(t_{0}\right)=0, \quad \eta\left(t_{1}\right)=0 .
$$

Note that if $a \in \mathcal{P}\left(\left[t_{0}, t_{1}\right], M\right)_{x}^{y}$ then

$$
\eta^{\mathbf{c}}\left(y^{\alpha}-\Psi^{\alpha}\left(x^{i}, y^{a}\right)\right) \circ a=0
$$

if and only if

$$
\frac{d \eta^{\alpha}}{d t}=\rho_{A}^{i} \eta^{A} \frac{\partial \Psi^{\alpha}}{\partial x^{i}}+\frac{d \eta^{a}}{d t} \frac{\partial \Psi^{\alpha}}{\partial y^{a}}+\mathcal{C}_{B A}^{a} y^{B} \eta^{A} \frac{\partial \Psi^{\alpha}}{\partial y^{a}}-\mathcal{C}_{B A}^{\alpha} y^{B} \eta^{A} .
$$


Let us look for the critical points of the action functional $\delta S: \mathcal{P}\left(\left[t_{0}, t_{1}\right], E\right)_{x}^{y} \rightarrow \mathbb{R}$

$$
\begin{aligned}
\delta S: \mathcal{P}\left(\left[t_{0}, t_{1}\right], E\right)_{x}^{y} & \rightarrow \mathbb{R} \\
a(t) & \mapsto \int_{t_{0}}^{t_{1}} L(a(t)) d t
\end{aligned}
$$

If we consider our infinitesimal variations then,

$$
\begin{aligned}
\frac{d}{d s} \int_{\mid s=0}^{t_{1}} L\left(a_{s}(t)\right) d t & =\int_{t_{0}}^{t_{1}} \frac{\partial L}{\partial x^{i}} \eta_{i}^{\mathbf{c}}+\frac{\partial L}{\partial y^{a}} \eta_{a}^{\mathbf{c}}+\frac{\partial L}{\partial y^{\alpha}} \frac{\partial \Psi^{\alpha}}{\partial x^{i}} \eta_{i}^{\mathbf{c}}+\frac{\partial L}{\partial y^{\alpha}} \frac{\partial \Psi^{\alpha}}{\partial y^{a}} \eta_{a}^{\mathbf{c}} d t \\
& =\int_{t_{0}}^{t_{1}} \frac{\partial \tilde{L}}{\partial x^{i}} \eta_{i}^{\mathbf{c}}+\frac{\partial \tilde{L}}{\partial y^{a}} \eta_{a}^{\mathbf{c}} d t \\
& =\int_{t_{0}}^{t_{1}} \frac{\partial \tilde{L}}{\partial x^{i}} \rho_{\alpha}^{i} \eta^{\alpha}+\frac{\partial \tilde{L}}{\partial x^{i}} \rho_{a}^{i} \eta^{a}+\frac{\partial \tilde{L}}{\partial y^{a}} \eta_{a}^{\mathbf{c}} d t
\end{aligned}
$$

Let $p_{\alpha}$ be the solution of the differential equations

$$
\dot{p}_{\alpha}=\left(\frac{\partial \tilde{L}}{\partial x^{i}}-p_{\beta} \frac{\partial \Psi^{\beta}}{\partial x^{i}}\right) \rho_{\alpha}^{i}-y^{a} \mathfrak{C}_{\alpha a}^{B} p_{B}-\Psi^{\beta} \mathfrak{C}_{\alpha \beta}^{B} p_{B},
$$

where

Using Eq. (5.9), we get that

$$
p_{a}=\frac{\partial \tilde{L}}{\partial y^{a}}-p_{\alpha} \frac{\partial \Psi^{\alpha}}{\partial y^{a}}
$$

$$
\begin{aligned}
\frac{d}{d t}\left(p_{\alpha} \eta^{\alpha}\right)= & \dot{p}_{\alpha} \eta^{\alpha}+p_{\alpha} \dot{\eta}^{\alpha} \\
= & p_{\alpha} \rho_{a}^{i} \eta^{a} \frac{\partial \Psi^{\alpha}}{\partial x^{i}}+p_{\alpha} \frac{d \eta^{a}}{d t} \frac{\partial \Psi^{\alpha}}{\partial y^{a}}+p_{\alpha} \mathcal{C}_{B A}^{a} y^{B} \eta^{A} \frac{\partial \Psi^{\alpha}}{\partial y^{a}}-p_{\alpha} \mathcal{C}_{B a}^{\alpha} y^{B} \eta^{a} \\
& +\frac{\partial \tilde{L}}{\partial x^{i}} \rho_{\alpha}^{i} \eta^{\alpha}-y^{a} \eta^{\alpha} \mathcal{C}_{\alpha a}^{b} p_{b}-\eta^{\alpha} \Psi^{\beta} \mathrm{C}_{\alpha \beta}^{b} p_{b} .
\end{aligned}
$$

If we use this equality, we deduce that

$$
\begin{aligned}
\frac{d}{d s}{ }_{\mid s=0} \int_{t_{0}}^{t_{1}} L\left(a_{s}(t)\right) d t= & \int_{t_{0}}^{t_{1}}\left(\frac{\partial \tilde{L}}{\partial x^{i}} \rho_{a}^{i} \eta^{a}+\frac{\partial \tilde{L}}{\partial y^{a}} \eta_{a}^{\mathbf{c}}+y^{a} \eta^{\alpha} \mathcal{C}_{\alpha a}^{b} p_{b}+\eta^{\alpha} \Psi^{\beta} \mathcal{C}_{\alpha \beta}^{b} p_{b}\right. \\
& -p_{\alpha} \rho_{a}^{i} \eta^{a} \frac{\partial \Psi^{\alpha}}{\partial x^{i}}+\frac{d}{d t}\left(p_{\alpha} \frac{\partial \Psi^{\alpha}}{\partial y^{a}}\right) \eta^{a}-p_{\alpha} \mathcal{C}_{B a}^{d} y^{B} \eta^{a} \frac{\partial \Psi^{\alpha}}{\partial y^{d}} \\
& \left.-p_{\alpha} \mathcal{C}_{B \beta}^{d} y^{B} \eta^{\beta} \frac{\partial \Psi^{\alpha}}{\partial y^{d}}+p_{\alpha} \mathcal{C}_{B a}^{\alpha} y^{B} \eta^{a}\right) d t .
\end{aligned}
$$

Finally, using Eq. (5.10) and the fact that

$$
\eta_{d}^{\mathbf{c}}=\frac{d \eta^{d}}{d t}+\left(\mathcal{C}_{b a}^{d} \eta^{a} y^{b}+\mathcal{e}_{a \alpha}^{d} \eta^{\alpha} y^{a}+\mathcal{e}_{\beta a}^{d} \eta^{a} \Psi^{\beta}+\mathcal{\complement}_{\beta \alpha}^{d} \eta^{\alpha} \Psi^{\beta}\right)
$$

we obtain that

$$
\begin{aligned}
& \frac{d}{d s}{ }_{\mid s=0} \int_{t_{0}}^{t_{1}} L\left(a_{s}(t)\right) d t= \\
& \quad \int_{t_{0}}^{t_{1}}\left[\left(\frac{\partial \tilde{L}}{\partial x^{i}}-p_{\alpha} \frac{\partial \Psi^{\alpha}}{\partial x^{i}}\right) \rho_{a}^{i}-\frac{d}{d t}\left(\frac{\partial \tilde{L}}{\partial y^{a}}-p_{\alpha} \frac{\partial \Psi^{\alpha}}{\partial y^{a}}\right)-y^{b} \bigodot_{a b}^{B} p_{B}-\Psi^{\alpha} \bigodot_{a \alpha}^{B} p_{B}\right] \eta^{a} d t
\end{aligned}
$$

Since the variations $\eta^{a}$ are free, we conclude that the equations are

$$
\left\{\begin{array}{l}
\dot{x}^{i}=y^{a} \rho_{a}^{i}+\Psi^{\alpha} \rho_{\alpha}^{i} \\
\dot{p}_{\alpha}=\left(\frac{\partial \tilde{L}}{\partial x^{i}}-p_{\beta} \frac{\partial \Psi^{\beta}}{\partial x^{i}}\right) \rho_{\alpha}^{i}-y^{a} \mathcal{C}_{\alpha a}^{B} p_{B}-\Psi^{\beta} \mathcal{C}_{\alpha \beta}^{B} p_{B} \\
\frac{d}{d t}\left(\frac{\partial \tilde{L}}{\partial y^{a}}-p_{\alpha} \frac{\partial \Psi^{\alpha}}{\partial y^{a}}\right)=\left(\frac{\partial \tilde{L}}{\partial x^{i}}-p_{\alpha} \frac{\partial \Psi^{\alpha}}{\partial x^{i}}\right) \rho_{a}^{i}-y^{b} \mathcal{C}_{a b}^{B} p_{B}-\Psi^{\alpha} \mathcal{C}_{a \alpha}^{B} p_{B}
\end{array}\right.
$$


with $p_{a}=\frac{\partial \tilde{L}}{\partial y^{a}}-p_{\alpha} \frac{\partial \Psi^{\alpha}}{\partial y^{a}}$, that is, we obtain the vakonomic equations for the vakonomic system $(L, M)$ on the Lie algebroid $\tau: E \rightarrow Q$.

\subsection{Examples.}

5.3.1. Skinner-Rusk formalism on Lie algebroids. Suppose that $\tau: E \rightarrow Q$ is a Lie algebroid, $L: E \rightarrow \mathbb{R}$ is a Lagrangian function and $M=E$, that is, we do not have constraints. Then,

$$
W_{0}=E^{*} \oplus E
$$

and the Pontryagin Hamiltonian $H_{W_{0}}: E^{*} \oplus E \rightarrow \mathbb{R}$ is locally given by

$$
H_{W_{0}}\left(x^{i}, p_{A}, y^{A}\right)=y^{A} p_{A}-L\left(x^{i}, y^{A}\right) \text {. }
$$

Let us apply the constraint algorithm. First, if $\Omega_{0}$ is the presymplectic section on $W_{0}$ given by $\Omega_{0}=\left(\mathcal{T p r}_{1}, \operatorname{pr}_{1}\right)^{*} \Omega_{E}$ then, since $\operatorname{Ker} \Omega_{0}=\operatorname{span}\left\{\mathcal{V}_{A}\right\}$, the primary constraint submanifold $W_{1}$ is locally characterized by

$$
p_{A}=\frac{\partial L}{\partial y^{A}}
$$

which are the definition of the momenta. $W_{1}$ is isomorphic to $E$ and the vakonomic equations reduce to

$$
\left\{\begin{array}{l}
\dot{x}^{i}=y^{A} \rho_{A}^{i}, \\
\frac{d}{d t}\left(\frac{\partial L}{\partial y^{A}}\right)=\rho_{A}^{i} \frac{\partial L}{\partial x^{i}}-\mathcal{C}_{A B}^{C} y^{B} p_{C} .
\end{array}\right.
$$

Using (5.11), it follows that Equations (5.12) are just the Euler-Lagrange equations for $L$ (see (2.9)). We remark that if $E=T Q$, this procedure is the Skinner-Rusk formulation of Lagrangian Mechanics (see [32, 33]). On the other hand, using Proposition 5.3. we deduce that the vakonomic system is regular if and only if the Lagrangian function $L$ is regular.

5.3.2. The tangent bundle. Let $E$ be the standard Lie algebroid $\tau_{T Q}: T Q \rightarrow Q$, $M \subseteq T Q$ be the constraint submanifold such that $\tau_{T Q \mid M}: M \rightarrow Q$ is a surjective submersion and $L: T Q \rightarrow \mathbb{R}$ be a standard Lagrangian function. Suppose that $\left(q^{A}, \dot{q}^{A}\right)=\left(q^{A}, \dot{q}^{a}, \dot{q}^{\alpha}\right)$ are local fibred coordinates on $T Q$ and that the submanifold $M$ is locally described by equations of the form

$$
\dot{q}^{\alpha}=\Psi^{\alpha}\left(q^{A}, \dot{q}^{a}\right) .
$$

As we know, the local structure functions of the Lie algebroid $\tau_{T Q}: T Q \rightarrow Q$ with respect to the local coordinates $\left(q^{A}, \dot{q}^{A}\right)$ are

$$
\rho_{A}^{B}=\delta_{A}^{B} \quad \text { and } \quad \mathcal{C}_{A B}^{C}=0 .
$$

Thus, if we apply the results of Sections 5.1 and 5.2 to this particular case we recover the geometric formulation of vakonomic mechanics developed in 9]. In particular, the vakonomic equations reduce to

$$
\left\{\begin{array}{l}
\dot{q}^{\alpha}=\Psi^{\alpha} \\
\frac{d}{d t}\left(\frac{\partial \tilde{L}}{\partial \dot{q}^{a}}\right)-\frac{\partial \tilde{L}}{\partial q^{a}}=\dot{p}_{\alpha} \frac{\partial \Psi^{\alpha}}{\partial \dot{q}^{a}}+p_{\alpha}\left[\frac{d}{d t}\left(\frac{\partial \Psi^{\alpha}}{\partial \dot{q}^{a}}\right)-\frac{\partial \Psi^{\alpha}}{\partial q^{a}}\right] \\
\dot{p}_{\alpha}=\frac{\partial \tilde{L}}{\partial q^{\alpha}}-p_{\beta} \frac{\partial \Psi^{\beta}}{\partial q^{\alpha}} .
\end{array}\right.
$$


5.3.3. Lie algebras. Let $\mathfrak{g}$ be a real Lie algebra of dimension $n$. Then, $\mathfrak{g}$ is a Lie algebroid over a single point.

Now, suppose that $\mathfrak{C}$ is an affine subspace of $\mathfrak{g}$ modelled over the vector space $C$ of dimension $n-\bar{m}$ and that $e_{0} \in \mathfrak{C}, e_{0} \neq 0$. We consider a basis $\left\{e_{A}\right\}=$ $\left\{e_{a}, e_{0}, e_{\bar{a}}\right\}=\left\{e_{a}, e_{\alpha}\right\}$ of $\mathfrak{g}$ such that $\left\{e_{a}\right\}$ is a basis of $C$ and

$$
\left[e_{A}, e_{B}\right]=\mathrm{C}_{A B}^{C} e_{C} .
$$

Denote by $\left(y^{a}, y^{0}, y^{\bar{a}}\right)=\left(y^{a}, y^{\alpha}\right)$ the linear coordinates on $\mathfrak{g}$ induced by the basis $\left\{e_{a}, e_{0}, e_{\bar{a}}\right\}=\left\{e_{a}, e_{\alpha}\right\}$. Then, $\mathfrak{C}$ is given by the equations

$$
y^{0}=1, \quad y^{\bar{a}}=0 .
$$

Next, assume that $L: \mathfrak{g} \rightarrow \mathbb{R}$ is a Lagrangian function and denote by $\tilde{L}: \mathfrak{C} \rightarrow \mathbb{R}$ the restriction of $L$ to $\mathfrak{C}$. Then, a curve

$$
\sigma: t \mapsto\left(y^{a}(t), y^{0}(t), y^{\bar{a}}(t)\right)=\left(y^{a}(t), 1,0, \ldots, 0\right)
$$

in $\mathfrak{C}$ is a solution of the vakonomic equations for the constrained system $(L, \mathfrak{C})$ if and only if

$$
\left\{\begin{array}{l}
\frac{d}{d t}\left(\frac{\partial \tilde{L}}{\partial y^{a}}\right)=-\frac{\partial \tilde{L}}{\partial y^{c}}\left(y^{b} \mathcal{C}_{a b}^{c}+\mathcal{C}_{a 0}^{c}\right)-p_{\beta}\left(y^{b} \mathcal{C}_{a b}^{\beta}+\mathcal{C}_{a 0}^{\beta}\right), \\
\dot{p}_{\alpha}=-\frac{\partial \tilde{L}}{\partial y^{c}}\left(y^{b} \mathcal{C}_{\alpha b}^{c}+\mathcal{C}_{\alpha 0}^{c}\right)-p_{\beta}\left(y^{b} \mathcal{C}_{\alpha b}^{\beta}+\mathcal{C}_{\alpha 0}^{\beta}\right) .
\end{array}\right.
$$

Now, we consider the curve $\gamma$ in $\mathfrak{g}^{*}$ whose components with respect to the dual basis $\left\{e^{A}\right\}$ are

$$
\gamma(t)=\left(\frac{\partial \tilde{L}}{\partial y^{a}} \underset{\mid \sigma(t)}{,}, p_{\alpha}(t)\right),
$$

that is, $\gamma$ is the sum of the curve $\frac{\partial \tilde{L}}{\partial y}$ and the curve

$$
t \mapsto \lambda(t) \in C^{o}
$$

whose components are $\lambda(t)=\left(0, p_{\alpha}(t)\right)$. Here, $C^{o} \subseteq \mathfrak{g}^{*}$ is the annihilator of the subspace $C$. Then, a direct computation, using (5.13), proves that $\gamma$ satisfies the Euler-Poincaré equations

$$
\frac{d}{d t}\left(\frac{\partial \tilde{L}}{\partial y}+\lambda\right)=a d_{\sigma}^{*}\left(\frac{\partial \tilde{L}}{\partial y}+\lambda\right) .
$$

This result is just the "Optimization Theorem for Nonholonomic Systems on Lie groups" which was proved in [21] (see Theorem 5.1 in [21]).

5.3.4. Atiyah algebroids and reduction in subriemannian geometry. Let $\pi: P \rightarrow Q$ be a principal bundle with structural group $G$. The dimension of $P$ (respectively, $Q$ ) is $n$ (respectively, $m$ ).

Suppose that $D$ is a distribution on $P$ such that

$$
T_{p} P=D_{p}+V_{p} \pi, \quad \text { for all } p \in P,
$$

where $V \pi$ is the vertical bundle to the principal bundle projection $\pi$.

We also suppose that $D$ is equipped with a bundle metric $\langle,\rangle_{D}$. Assuming that both $D$ and $\langle,\rangle_{D}$ are $G$-invariant, then one may construct a nonholonomic connection as follows (see [4]).

We will suppose that the space

$$
S_{p}=D_{p} \cap V_{p} \pi
$$

has constant dimension $r$, for all $p \in P$. Under this condition, the horizontal space of the nonholonomic connection at the point $p$ is $S_{p}^{\perp}$, where $S_{p}^{\perp}$ is the orthogonal 
complement of $S_{p}$ on $D$ with respect to the bundle metric $\langle,\rangle_{D}$. We will denote by $\omega^{n h}: T P \rightarrow \mathfrak{g}$ the corresponding Lie algebra-valued 1-form.

Define a Lagrangian function on $D$ by $L\left(v_{q}\right)=\frac{1}{2}\left\langle v_{q}, v_{q}\right\rangle_{D}$ where $v_{q} \in D_{q}$.

Now, we consider the Atiyah algebroid $\tau_{P} \mid G: T P / G \rightarrow Q=P / G$ associated with the principal bundle $\pi: P \rightarrow Q=P / G$. Note that the space of orbits $D / G$ of the action of $G$ on $D$ is a vector subbundle (over $Q$ ) of the Atiyah algebroid.

Next, we will obtain a local basis of $\Gamma(T P / G)$ adapted to the vector subbundle $D / G$.

For this purpose, we choose a local trivialization of the principal bundle $\pi: P \rightarrow$ $Q=P / G$ to be $U \times G$, where $U$ is an open subset of $Q$. Let $e$ be the identity element of $G$ and assume that there are local coordinates $\left(x^{i}\right)$ on $U$. If $\left(\frac{\partial}{\partial x^{i}}\right)^{h}$ is the horizontal lift of the vector field $\frac{\partial}{\partial x^{i}}$ on $U$ then $\left(\frac{\partial}{\partial x^{i}}\right)^{h}$ is a $G$-invariant vector field and $\left\{\left(\frac{\partial}{\partial x^{i}}\right)^{h}\right\}_{i=1, \ldots, m}$ is a local basis of $S^{\perp}$.

On the other hand, we consider a family of smooth maps

$$
\tilde{e}_{A}: U \rightarrow \mathfrak{g}, \quad A=1, \ldots, n-m,
$$

such that, for every $q \in U,\left\{\tilde{e}_{A}(q)\right\}$ is a basis of $\mathfrak{g}$ and $\left\{\tilde{e}_{a}(q)\right\}_{a=1, \ldots, r}$ is a basis of the vector space

$$
\mathfrak{g}^{(q, e)}=\left\{\xi \in \mathfrak{g} \mid \xi_{P}(q, e) \in D_{(q, e)}\right\} .
$$

Here, $\xi_{P}$ is the infinitesimal generator of the free action of $G$ on $P$ associated with $\xi \in \mathfrak{g}$.

Now, we introduce the vertical vector fields on $U \times G$ given by

$$
\begin{aligned}
V_{A}: U \times G & \rightarrow T(U \times G) \cong T U \times T G \\
(q, g) & \mapsto \overleftarrow{\tilde{e}_{A}(q)}(g)
\end{aligned}
$$

$\overleftarrow{\overleftarrow{e}_{A}(q)}$ is the left-invariant vector field on $G$ induced by the element $\tilde{e}_{A}(q)$ of $\mathfrak{g}$. Then, it is clear that

$$
\left\{\left(\frac{\partial}{\partial x^{i}}\right)^{h}, V_{A}\right\}=\left\{\left(\frac{\partial}{\partial x^{i}}\right)^{h}, V_{a}, V_{\alpha}\right\}
$$

is a local basis of $G$-invariant vector fields on $P$ and

$$
\left\{\left(\frac{\partial}{\partial x^{i}}\right)^{h}, V_{a}\right\}
$$

is a local basis of the space of sections of the vector subbundle $D \rightarrow P$. Thus, we have the corresponding local basis of sections

$$
\left\{e_{i}, e_{A}\right\}=\left\{e_{i}, e_{a}, e_{\alpha}\right\}
$$

of the Atiyah algebroid $\tau_{P} \mid G: T P / G \rightarrow Q=P / G$ which is adapted to the vector subbundle $D / G \rightarrow Q=P / G$.

Denote by $\left(x^{i}, \dot{x}^{i}, y^{a}, y^{\alpha}\right)$ the local coordinates on $T P / G$ induced by the basis $\left\{e_{i}, e_{a}, e_{\alpha}\right\}$. Then $D / G$ is locally characterized by the equations

$$
y^{\alpha}=0 \text {. }
$$

On the other hand, if

$$
\begin{aligned}
& \omega^{n h}\left(\frac{\partial}{\partial x^{i}}\right)=\Gamma_{i}^{A}(q) e_{A}(q), \\
& {\frac{\partial \tilde{e}_{B}}{\partial x^{i}}{ }_{\mid q}}=\chi_{i B}^{C}(q) \tilde{e}_{C}(q), \quad\left[\tilde{e}_{A}(q), \tilde{e}_{B}(q)\right]=\mathcal{C}_{A B}^{C} \tilde{e}_{C}(q),
\end{aligned}
$$

for all $q \in U$, it follows that

$$
\left[e_{i}, e_{j}\right]=\mathcal{B}_{i j}^{C} e_{C}, \quad\left[e_{i}, e_{B}\right]=\mu_{i B}^{C} e_{C}, \quad\left[e_{A}, e_{B}\right]=\mathcal{C}_{A B}^{C} e_{C},
$$


where

$$
\begin{aligned}
\mathcal{B}_{i j}^{C} & =\frac{\partial \Gamma_{i}^{C}}{\partial x^{j}}-\frac{\partial \Gamma_{j}^{C}}{\partial x^{i}}+\Gamma_{i}^{A} \Gamma_{j}^{B} \mathcal{C}_{A B}^{C}+\Gamma_{i}^{A} \chi_{j A}^{C}-\Gamma_{j}^{A} \chi_{i A}^{C}, \\
\mu_{i B}^{C} & =\chi_{i B}^{C}-\Gamma_{i}^{A} \mathcal{C}_{A B}^{C} .
\end{aligned}
$$

Moreover, if $\rho: T P / G \rightarrow T Q$ is the anchor map of the Atiyah algebroid $\tau_{P} \mid G$ : $T P / G \rightarrow Q=P / G$, we have that

$$
\rho\left(e_{i}\right)=\frac{\partial}{\partial x^{i}}, \quad \rho\left(e_{A}\right)=0 .
$$

Since the bundle metric $\langle,\rangle_{D}$ is $G$-invariant, it induces a bundle metric $\langle,\rangle_{D / G}$ on $D / G$, with associated lagrangian function $l: D / G \rightarrow \mathbb{R}$. Therefore, we deduce that a curve

$$
\sigma: t \mapsto\left(x^{i}(t), \dot{x}^{i}(t), y^{a}(t), y^{\alpha}(t)\right)=\left(x^{i}(t), \dot{x}^{i}(t), y^{a}(t), 0\right)
$$

in $D / G$ is a solution of the vakonomic equations for the constrained system $(l, D / G)$ if and only if

$$
\left\{\begin{array}{l}
\frac{d}{d t}\left(\frac{\partial l}{\partial \dot{x}^{i}}\right)=\frac{\partial l}{\partial x^{i}}-\left(\dot{x}^{j} \mathcal{B}_{i j}^{a}+y^{b} \mu_{i b}^{a}\right) \frac{\partial l}{\partial y^{a}}-\left(\dot{x}^{j} \mathcal{B}_{i j}^{\alpha}+y^{b} \mu_{i b}^{\alpha}\right) p_{\alpha} \\
\frac{d}{d t}\left(\frac{\partial l}{\partial y^{a}}\right)=\left(\dot{x}^{j} \mu_{j a}^{c}-y^{b} \mathcal{C}_{a b}^{c}\right) \frac{\partial l}{\partial y^{c}}+\left(\dot{x}^{j} \mu_{j a}^{\alpha}-y^{b} \mathcal{C}_{a b}^{\alpha}\right) p_{\alpha} \\
\dot{p}_{\alpha}=\left(\dot{x}^{i} \mu_{i \alpha}^{b}-y^{a} \mathcal{C}_{\alpha a}^{b}\right) \frac{\partial l}{\partial y^{b}}+\left(\dot{x}^{i} \mu_{i \alpha}^{\beta}-y^{a} \mathcal{C}_{\alpha a}^{\beta}\right) p_{\beta}
\end{array}\right.
$$

Observe that if the basis of sections of $D / G,\left\{e_{i}, e_{a}\right\}$, is orthonormal then

$$
l\left(x^{i}, \dot{x}^{i}, y^{a}\right)=\frac{1}{2}\left(\sum_{i}\left(\dot{x}^{i}\right)^{2}+\sum_{a}\left(y^{a}\right)^{2}\right)
$$

and the vakonomic equations are:

$$
\left\{\begin{array}{l}
\ddot{x}^{i}=-\sum_{a}\left(\dot{x}^{j} \mathcal{B}_{i j}^{a}+y^{b} \mu_{i b}^{a}\right) y^{a}-\left(\dot{x}^{j} \mathcal{B}_{i j}^{\alpha}+y^{b} \mu_{i b}^{\alpha}\right) p_{\alpha} \\
\dot{y}^{a}=\sum_{c}\left(\dot{x}^{j} \mu_{j a}^{c}-y^{b} \mathcal{C}_{a b}^{c}\right) y^{c}+\left(\dot{x}^{j} \mu_{j a}^{\alpha}-y^{b} \mathcal{C}_{a b}^{\alpha}\right) p_{\alpha} \\
\dot{p}_{\alpha}=\sum_{b}\left(\dot{x}^{i} \mu_{i \alpha}^{b}-y^{a} \mathcal{C}_{\alpha a}^{b}\right) y^{b}+\left(\dot{x}^{i} \mu_{i \alpha}^{\beta}-y^{a} \mathcal{C}_{\alpha a}^{\beta}\right) p_{\beta} .
\end{array}\right.
$$

Of course, it is also possible to study the abnormal solutions taking the lagrangian $l \equiv 0$.

Example 5.11. (See [19, 31]). As a simple but illustrative example, consider $\mathbb{R}^{3}$ with the distribution $D=\operatorname{ker} \omega$ where $\omega$ is the Martinet 1-form: $\omega=d x^{3}-\frac{\left(x^{1}\right)^{2}}{2} d x^{2}$. Consider the vector fields generating $D$ :

$$
\frac{\partial}{\partial x^{2}}+\frac{\left(x^{1}\right)^{2}}{2} \frac{\partial}{\partial x^{3}}, \quad \frac{\partial}{\partial x^{1}},
$$

and the bundle metric $\langle,\rangle_{D}$ which makes both vector fields orthonormal.

Take now the action by translations:

$$
\begin{aligned}
& \mathbb{R}^{2} \times \mathbb{R}^{3} \longrightarrow \mathbb{R}^{3} \\
& \left((a, b),\left(x^{1}, x^{2}, x^{3}\right)\right) \longmapsto\left(x^{1}, x^{2}+a, x^{3}+b\right) .
\end{aligned}
$$

We have a principal bundle structure $\pi: \mathbb{R}^{3} \rightarrow \mathbb{R}^{3} / \mathbb{R}^{2} \equiv \mathbb{R}$ being both $D$ and $\langle,\rangle_{D}$ $\mathbb{R}^{2}$-invariant. 
Observe that $V \pi=\operatorname{span}\left\{\frac{\partial}{\partial x^{2}}, \frac{\partial}{\partial x^{3}}\right\}, S=D \cap V \pi=\operatorname{span}\left\{V_{1}=\frac{\partial}{\partial x^{2}}+\frac{\left(x^{1}\right)^{2}}{2} \frac{\partial}{\partial x^{3}}\right\}$ and $S^{\perp}=\operatorname{span}\left\{\frac{\partial}{\partial x^{1}}\right\}$.

On the Atiyah algebroid $T \mathbb{R}^{3} / \mathbb{R}^{2} \cong \mathbb{R} \times \mathbb{R}^{3} \rightarrow \mathbb{R}$ we have the induced basis of sections $e_{i}: \mathbb{R} \rightarrow \mathbb{R} \times \mathbb{R}^{3}, i=1,2,3$ :

$$
\begin{aligned}
& e_{1}(x)=(x ;(1,0,0)), \\
& e_{2}(x)=\left(x ;\left(0,1, \frac{x^{2}}{2}\right)\right) \\
& e_{3}(x)=(x ;(0,0,1))
\end{aligned}
$$

which induces coordinates $\left(x, \dot{x}, y^{1}, y^{2}\right)$. Then, $D / \mathbb{R}^{2}$ is characterized by the equation $y^{2}=0$.

Observe that

$$
\left[e_{1}, e_{2}\right]=x e_{3}, \quad\left[e_{1}, e_{3}\right]=0, \quad\left[e_{2}, e_{3}\right]=0
$$

and

$$
l\left(x ; \dot{x}, y^{1}\right)=\frac{1}{2}\left((\dot{x})^{2}+\left(y^{1}\right)^{2}\right)
$$

Consequently the vakonomic equations are:

$$
\begin{aligned}
\dot{p}_{2} & =0 \\
\ddot{x} & =-y^{1} x p_{2} \\
\dot{y}^{1} & =\dot{x} x p_{2} .
\end{aligned}
$$

That is, $p_{2}=k$ constant, and the vakonomic equations are precisely,

$$
\begin{aligned}
\ddot{x} & =-k y^{1} x \\
\dot{y}^{1} & =k \dot{x} x .
\end{aligned}
$$

Since $\frac{1}{2}\left(\dot{x}^{2}+\left(y^{1}\right)^{2}\right)$ is a constant of motion then we can take $\dot{x}(t)=r \sin \theta(t)$ and $y^{1}(t)=r \cos \theta(t)$ where $\theta(t)$ verifies the equation of pendulum $\ddot{\theta}(t)=-k r \sin \theta(t)$ (see also Example 5.12).

5.3.5. Optimal Control on Lie algebroids as vakonomic systems. (See [10, 29]). Let $\tau: E \rightarrow Q$ be a Lie algebroid and $C$ be a manifold fibred over the state manifold $\pi: C \rightarrow Q$. We also consider a section $\sigma: C \rightarrow E$ along $\pi$ and an index function $l: C \rightarrow \mathbb{R}$.

One important case happens when the section $\sigma: C \rightarrow E$ along $\pi$ is an embedding, in this case, the image $M=\sigma(C)$ is a submanifold of $E$. Moreover, since $\sigma: C \longrightarrow M$ is a diffeomorphism, we can define $L: M \longrightarrow \mathbb{R}$ by $L=l \circ \sigma^{-1}$. In conclusion, it is equivalent to analyze the optimal control defined by $(l, \sigma)$ (applying the Pontryaguin maximum principle) that to study the vakonomic problem on the Lie algebroid $\tau: E \rightarrow Q$ defined by $(L, M)$.

More generally (without assuming the embedding condition), we can construct the prolongation $\tau^{\pi}: \mathcal{T}^{E} C \rightarrow C$ of the Lie algebroid $\tau: E \rightarrow Q$ over the smooth map $\pi: C \rightarrow Q$, that is

$$
\mathcal{T}^{E} C=\left\{\left(e, X_{p}\right) \in E_{\pi(p)} \times T_{p} C \mid \rho(e)=T \pi\left(X_{p}\right)\right\} .
$$

Moreover, we have the constraint submanifold $M$ characterized by

$$
M=\left\{\left(e, X_{p}\right) \in \mathcal{T}_{p}^{E} C \mid \sigma(p)=e\right\}
$$

and the lagrangian function $L: \mathcal{T}^{E} C \rightarrow \mathbb{R}$ given by $L=l \circ \tau^{\pi}$. This is the vakonomic system associated with the optimal control system. If $E=T Q$ is the tangent bundle of the state space $Q$, it is not difficult to show that the prolongation of $T Q$ along 
$\pi: C \rightarrow Q$ is just the tangent bundle $T C$. Under this isomorphism, the constraint submanifold is

$$
M=\left\{X \in T C \mid T \pi(X)=\sigma\left(\tau_{C}(X)\right)\right\} .
$$

Thus, we recover the construction in [10], Section 4.

Example 5.12. Consider the following mechanical problem [8, 20, 21]. A (homogeneous) sphere of radius $r=1$, mass $m$ and inertia about any axis $k^{2}$, rolls without sliding on a horizontal table which rotates with constant angular velocity $\Omega$ about the $x^{3}$-axis. The coordinates of the point of contact of the sphere with the plane are $\left(x^{1}, x^{2}\right)$. The configuration space of the sphere is $Q=\mathbb{R}^{2} \times S O(3)$ and the Lagrangian of the system corresponds to the kinetic energy

$$
K\left(x^{1}, x^{2} ; \dot{x}^{1}, \dot{x}^{2}, \omega_{x^{1}}, \omega_{x^{2}}, \omega_{x^{3}}\right)=\frac{1}{2}\left(m\left(\dot{x}^{1}\right)^{2}+m\left(\dot{x}^{2}\right)^{2}+m k^{2}\left(\omega_{x^{1}}^{2}+\omega_{x^{2}}^{2}+\omega_{x^{3}}^{2}\right)\right),
$$

where $\left(\omega_{x^{1}}, \omega_{x^{2}}, \omega_{x^{3}}\right)$ are the components of the angular velocity of the sphere.

Since the ball is rolling without sliding on a rotating table then the system is subjected to the affine constraints:

$$
\begin{aligned}
& \dot{x}^{1}-\omega_{x^{2}}=-\Omega x^{2}, \\
& \dot{x}^{2}+\omega_{x^{1}}=\Omega x^{1},
\end{aligned}
$$

where $\Omega$ is constant. Moreover, it is clear that $Q=\mathbb{R}^{2} \times S O(3)$ is the total space of a trivial principal $S O(3)$-bundle over $\mathbb{R}^{2}$ and the bundle projection $\phi: Q \rightarrow \mathbb{R}^{2}$ is just the canonical projection on the first factor. Therefore, we may consider the corresponding Atiyah algebroid $T Q / S O(3)$ over $\mathbb{R}^{2}$.

Since the Atiyah algebroid $T Q / S O(3)$ is isomorphic to the product manifold $T \mathbb{R}^{2} \times \mathfrak{s o}(3) \cong T \mathbb{R}^{2} \times \mathbb{R}^{3}$, then a section of $T Q / S O(3) \cong T \mathbb{R}^{2} \times \mathbb{R}^{3} \rightarrow \mathbb{R}^{2}$ is a pair $(X, u)$, where $X$ is a vector field on $\mathbb{R}^{2}$ and $u: \mathbb{R}^{2} \rightarrow \mathbb{R}^{3}$ is a smooth map. Therefore, a global basis of sections of $T \mathbb{R}^{2} \times \mathbb{R}^{3} \rightarrow \mathbb{R}^{2}$ is

$$
e_{1}=\left(\frac{\partial}{\partial x^{1}}, 0\right), e_{2}=\left(\frac{\partial}{\partial x^{2}}, 0\right), e_{3}=\left(0, E_{1}\right), e_{4}=\left(0, E_{2}\right), e_{5}=\left(0, E_{3}\right) .
$$

where $\left\{E_{1}, E_{2}, E_{3}\right\}$ is the canonical basis on $\mathbb{R}^{3}$.

The anchor map $\rho: T \mathbb{R}^{2} \times \mathbb{R}^{3} \rightarrow T \mathbb{R}^{2}$ is the projection over the first factor and if $\llbracket \cdot, \cdot \rrbracket$ is the Lie bracket on the space $\operatorname{Sec}(T Q / S O(3))$ then the only non-zero fundamental Lie brackets are

$$
\llbracket e_{3}, e_{4} \rrbracket=e_{5}, \quad \llbracket e_{4}, e_{5} \rrbracket=e_{3}, \quad \llbracket e_{5}, e_{3} \rrbracket=e_{4} .
$$

It is clear that the Lagrangian and the nonholonomic constraints are defined on the Atiyah algebroid $T Q / S O(3)$ (since the system is $S O(3)$-invariant). In fact, we have a nonholonomic system on the Atiyah algebroid $T Q / S O(3) \cong T \mathbb{R}^{2} \times \mathbb{R}^{3}$. This kind of systems was recently analyzed by J. Cortés et al [8] (in particular, this example was carefully studied).

After some computations the equations of motion for this nonholonomic system are precisely

$$
\left.\begin{array}{rl}
\dot{x}^{1}-\omega_{x^{2}} & =-\Omega x^{2}, \\
\dot{x}^{2}+\omega_{x^{1}} & =\Omega x^{1}, \\
\omega_{x^{3}} & =c
\end{array}\right\}
$$

where $c$ is a constant, together with

$$
\begin{aligned}
& \ddot{x}^{1}+\frac{k^{2} \Omega}{1+k^{2}} \dot{x}^{2}=0 \\
& \ddot{x}^{2}-\frac{k^{2} \Omega}{1+k^{2}} \dot{x}^{1}=0
\end{aligned}
$$


Now, we pass to an optimization problem. Assume full control over the motion of the center of the ball (the shape variables) and consider the cost function

$$
L\left(x^{1}, x^{2} ; \dot{x}^{1}, \dot{x}^{2}, \omega_{x^{1}}, \omega_{x^{2}}, \omega_{x^{3}}\right)=\frac{1}{2}\left(\left(\dot{x}^{1}\right)^{2}+\left(\dot{x}^{2}\right)^{2}\right),
$$

and the following optimal control problem:

PLATE-BALL PROBLEM [21]. Given points $q_{0}, q_{1} \in Q$, find an optimal control curve $\left(x^{1}(t), x^{2}(t)\right)$ on the reduced space that steer the system from $q_{0}$ to $q_{1}$, minimizes $\int_{0}^{1} \frac{1}{2}\left(\left(\dot{x}^{1}\right)^{2}+\left(\dot{x}^{2}\right)^{2}\right) d t$, subject to the constraints defined by Equations (5.14).

Observe that the Plate-Ball problem is equivalent to the optimal control problem given by the section $\sigma: \mathbb{R}^{2} \times \mathbb{R}^{2} \rightarrow T \mathbb{R}^{2} \times \mathbb{R}^{3}$ along $T \mathbb{R}^{2} \times \mathbb{R}^{3} \rightarrow \mathbb{R}^{2}$ given by

$$
\sigma\left(x^{1}, x^{2} ; u^{1}, u^{2}\right)=\left(x^{1}, x^{2} ; u^{1}, u^{2},-u^{2}+\Omega x^{1}, u^{1}+\Omega x^{2}, c\right) .
$$

and index function $l\left(x^{1}, x^{2} ; u^{1}, u^{2}\right)=\frac{1}{2}\left(\left(u^{1}\right)^{2}+\left(u^{2}\right)^{2}\right)$. Since $\sigma$ is obviously an embedding this is equivalent to the proposed Plate-Ball problem.

A necessary condition for optimality of the Plate-Ball problem is given by the corresponding vakonomic equations. For coherence with the notation introduced along this paper, denote by

$$
y^{1}=\dot{x}^{1}, y^{2}=\dot{x}^{2}, y^{3}=\omega_{x^{1}}, y^{4}=\omega_{x^{2}}, y^{5}=\omega_{x^{3}}
$$

Therefore, the vakonomic problem is given by the Lagrangian $L=\frac{1}{2}\left(\left(y^{1}\right)^{2}+\left(y^{2}\right)^{2}\right)$ and the submanifold $M$ defined by the constraints:

$$
\begin{aligned}
& y^{3}=\Psi^{3}\left(x^{1}, x^{2}, y^{1}, y^{2}\right)=-y^{2}+\Omega x^{1} \\
& y^{4}=\Psi^{4}\left(x^{1}, x^{2}, y^{1}, y^{2}\right)=y^{1}+\Omega x^{2} \\
& y^{5}=\Psi^{5}\left(x^{1}, x^{2}, y^{1}, y^{2}\right)=c
\end{aligned}
$$

After simple computations we obtain that the vakonomic equations are:

$$
\begin{aligned}
\dot{p}_{3} & =c p_{4}-\left(y^{1}+\Omega x^{2}\right) p_{5} \\
\dot{p}_{4} & =-c p_{3}-\left(y^{2}-\Omega x^{1}\right) p_{5} \\
\dot{p}_{5} & =\left(y^{1}+\Omega x^{2}\right) p_{3}+\left(y^{2}-\Omega x^{1}\right) p_{4} \\
\frac{d}{d t}\left(y^{1}-p_{4}\right) & =-\Omega p_{3} \\
\frac{d}{d t}\left(y^{2}+p_{3}\right) & =-\Omega p_{4} \\
y^{1}=\dot{x}^{1}, & y^{2}=\dot{x}^{2} .
\end{aligned}
$$

The system is obviously regular since the matrix

$$
\left(\frac{\partial^{2} L}{\partial y^{a} \partial y^{b}}-\sum_{\alpha=3}^{5} p_{\alpha} \frac{\partial^{2} \Psi^{\alpha}}{\partial y^{a} \partial y^{b}}\right)_{1 \leq a, b \leq 2}=\left(\begin{array}{ll}
1 & 0 \\
0 & 1
\end{array}\right)
$$

is non-singular. Therefore, there exists a unique solution of the vakonomic equations on $W_{1}$, determined by the conditions:

$$
\begin{aligned}
& p_{1}=\frac{\partial L}{\partial y^{1}}-p_{\alpha} \frac{\partial \Psi^{\alpha}}{\partial y^{1}}=y^{1}-p_{4} \\
& p_{2}=\frac{\partial L}{\partial y^{2}}-p_{\alpha} \frac{\partial \Psi^{\alpha}}{\partial y^{2}}=y^{2}+p_{3}
\end{aligned}
$$

Therefore, it follows that $\left(x^{1}, x^{2}, p_{1}, p_{2}, p_{3}, p_{4}, p_{5}\right)$ are local coordinates on $W_{1}$ (or, from Corollary 5.9 , on $E^{*}$, if you prefer). 
Moreover, on $W_{1}$ we have a well-defined Poisson bracket $\{,\}_{(L, M)}$ whose nonvanishing terms are:

$$
\begin{gathered}
\left\{x^{1}, p_{1}\right\}_{(L, M)}=1,\left\{x^{2}, p_{2}\right\}_{(L, M)}=1 \\
\left\{p_{3}, p_{4}\right\}_{(L, M)}=-p_{5},\left\{p_{3}, p_{5}\right\}_{(L, M)}=p_{4},\left\{p_{4}, p_{5}\right\}_{(L, M)}=-p_{3}
\end{gathered}
$$

In these coordinates the Hamiltonian $H_{W_{1}}$ is:

$$
H_{W_{1}}\left(x^{1}, x^{2}, p_{1}, p_{2}, p_{3}, p_{4}, p_{5}\right)=\frac{1}{2}\left(p_{1}+p_{4}\right)^{2}+\frac{1}{2}\left(p_{2}-p_{3}\right)^{2}+c p_{5}+p_{3} \Omega x^{1}+p_{4} \Omega x^{2}
$$

and the vakonomic equations are

$$
\begin{aligned}
& \dot{p}_{1}=\left\{p_{1}, H_{W_{1}}\right\}_{(L, M)}=-\Omega p_{3} \\
& \dot{p}_{2}=\left\{p_{2}, H_{W_{1}}\right\}_{(L, M)}=-\Omega p_{4} \\
& \dot{p}_{3}=\left\{p_{3}, H_{W_{1}}\right\}_{(L, M)}=c p_{4}-\left(p_{1}+p_{4}+\Omega x^{2}\right) p_{5} \\
& \dot{p}_{4}=\left\{p_{4}, H_{W_{1}}\right\}_{(L, M)}=-c p_{3}-\left(p_{2}-p_{3}-\Omega x^{1}\right) p_{5} \\
& \dot{p}_{5}=\left\{p_{5}, H_{W_{1}}\right\}_{(L, M)}=\left(p_{1}+p_{4}+\Omega x^{2}\right) p_{3}+\left(p_{2}-p_{3}-\Omega x^{1}\right) p_{4} \\
& \dot{x}^{1}=\left\{x^{1}, H_{W_{1}}\right\}_{(L, M)}=p_{1}+p_{4} \\
& \dot{x}^{2}=\left\{x^{2}, H_{W_{1}}\right\}_{(L, M)}=p_{2}-p_{3}
\end{aligned}
$$

One of the most studied cases is when $c=0$ (the sphere is rolled that its angular velocity is always parallel to the horizontal plane) and $\Omega=0$ (not rotation of the plane). In this case the equations reduced to

$$
\begin{gathered}
\dot{p}_{3}=-y^{1} p_{5}, \dot{p}_{4}=-y^{2} p_{5}, \dot{p}_{5}=y^{1} p_{3}+y^{2} p_{4} \\
\frac{d}{d t}\left(y^{1}-p_{4}\right)=0, \frac{d}{d t}\left(y^{2}+p_{3}\right)=0 \\
y^{1}=\dot{x}^{1}, y^{2}=\dot{x}^{2} .
\end{gathered}
$$

From these equations it is easy to deduce that

$$
\frac{d}{d t}\left(\left(y^{1}\right)^{2}+\left(y^{2}\right)^{2}\right)=0
$$

Therefore $y^{1}(t)=\cos \theta(t)$ and $y^{2}(t)=\sin \theta(t)$, when $1=\sqrt{\left(y^{1}\right)^{2}+\left(y^{2}\right)^{2}}$. Moreover, it is easy to deduce that $p_{5}=\dot{\theta}$.

Now, since $y^{1}=p_{4}+k_{1}$ and $y^{2}=-p_{3}+k_{2}$ with $k_{1}, k_{2} \in \mathbb{R}$ constants, taking $k_{1}=r \cos \varphi$ and $k_{2}=r \sin \varphi$ then from Equation $\dot{p}_{5}=y^{1} p_{3}+y^{2} p_{4}$ we deduce that

$$
\ddot{\theta}=r \cos \theta \sin \varphi-r \sin \theta \cos \varphi=-r \sin (\theta-\varphi)
$$

that is, the angle $\theta$ satisfies the equation of pendulum, while the coordinates of the contact point satisfy the ODEs: $\dot{x}^{1}=\cos \theta$ and $\dot{x}^{2}=\sin \theta$. The remarkable result is that these equations say that the contact point of the sphere rolling optimally traces an Euler elastica (see [18).

\section{Conclusions And future WORK}

We have developed a general geometrical setting for constrained mechanical systems in the context of Lie algebroids. We list the main results obtained in this paper:

- We develop a constraint algorithm for presymplectic Lie algebroids and discuss the reduction of presymplectic Lie algebroids. 
- For a singular Lagrangian function on a Lie algebroid, we look for solutions of the corresponding dynamical equation, applying the previously introduced constraint algorithm. In addition, we find the submanifolds where the solution is a SODE (a second order differential equation). The theory is illustrated with an example.

- We study vakonomic mechanics on Lie algebroids. As for singular Lagrangian systems, we deduce the vakonomic equations by means of a constraint algorithm. We define the vakonomic bracket in this setting. Furthermore, the variational point of view and some explicit examples are discussed. We remark that we just look for the so-called normal solutions. We postpone for a future work a detailed variational analysis of vakonomic mechanics on Lie algebroids, including abnormal solutions... We will illustrate our theory with an example related with Optimal Control Theory, but our approach may cover other interesting examples (see [3, 15]) and admits, in a standard way, variational discretizations (see [2, 17]).

\section{ACKNOWLEDGMENTS}

This work has been partially supported by MEC (Spain) Grants MTM 200603322, MTM 2007-62478, project "Ingenio Mathematica" (i-MATH) No. CSD 2006-00032 (Consolider-Ingenio 2010) and S-0505/ESP/0158 of the CAM. D. Iglesias wants to thank MEC for a Research Contract "Juan de la Cierva".

\section{REFERENCES}

[1] V.I. Arnold: Dynamical Systems, Vol. III, Springer-Verlag, New York, Heidelberg, Berlin, 1998.

[2] R. Benito, D. Martín de Diego: Discrete vakonomic Mechanics, J. Math. Phys., 46 (8) (2005), 083521-083538.

[3] A.M. Bloch : Nonholonomic Mechanics and Control, Interdisciplinary Applied Mathematics Series 24, Springer-Verlag New-York, 2003.

[4] A. M. Bloch, P.S. Krishnaprasad, J. E. Marsden, R. M. Murray: Nonholonomic Mechanical Systems with Symmetry, Arch. Rational Mech. Anal. 136 (1996) 21-99.

[5] M. Crainic, R.L. Fernandes: Integrability of Lie brackets, Ann. of Math. 157 (2003) 575-620.

[6] A. Capri, M. Kobayashi: A mechanical model with constraints, J. Math. Phys. 23 (1982), 736-741.

[7] A. Capri, M. Kobayashi: The first-rank tensor field coupled to an electromagnetic field, $J$. Phys. A Math. Gen. 26 (1987) 6101-6112.

[8] J. Cortés, M. de León, J.C. Marrero, E. Martínez: Nonholonomic Lagrangian systems on Lie algebroids, Preprint (2005), math-ph/0512003

[9] J. Cortés, M. de León, D. Martín de Diego, S. Martínez: Geometric description of vakonomic and nonholonomic dynamics. Comparison of solutions, SIAM J. Control Optim. 41 (2002) $1389-1412$.

[10] J. Cortés, M. de León, D. Martín de Diego, S. Martínez: General symmetries in optimal control. Rep. Math. Phys. 53 (2004) 55-78.

[11] M.J. Gotay, Presymplectic manifolds, geometric constraint theory and the Dirac-Bergmann theory of constraints, Dissertation, Center for Theoretical Physics, University of Maryland, 1979.

[12] M.J. Gotay, J. Nester, Presymplectic Lagrangian systems II: the second order differential equation problem. Ann. Inst. Henri Poincaré A 32 (1980), 1-13.

[13] M. Gotay, J. M. Nester, G. Hinds: Presymplectic manifolds and the Dirac-Bergmann theory of constraints. J. Math. Phys. 19 (1978), no. 11, 2388-2399.

[14] K. Grabowska, P. Urbański, J. Grabowski: Geometrical mechanics on algebroids. Int. J. Geom. Methods Mod. Phys. 3 (3) (2006), 559-575.

[15] I. Hussein, A. M. Bloch: Optimal Control of Underactuated Nonholonomic Mechanical Systems. Proceedings of the 2006 American Control Conference, Minneapolis, Minnesota, USA, (2006), 5590-5595.

[16] L.A. Ibort, C. López Lacasta: On the existence of local and global Lagrangians for ordinary differential equations. J. Phys. A 23 (1990), no. 21, 4779-4792. 
[17] D. Iglesias-Ponte, J.C. Marrero, D. Martín de Diego, E. Martínez: Discrete Nonholonomic Lagrangian Systems on Lie Groupoids, to appear in Journal of Nonlinear Science, arXiv:0704.1543v1 [math.DG].

[18] V. Jurdjevic: Geometric Control Theory, Cambridge Studie in Advances Mathematics 51, Cambridge University Press 1997.

[19] V. Jurdjevic. R.W. Sharpe (Editors). Geometric Control and Non-holonomic Mechanics, Conference on Geometric Control and Non-holonomic Mechanics, June 19-21, 1996, Mexico City, CMS Conference Proceedings 25, American Mathematical Society, 1998.

[20] W-S. Koon: Reduction, Reconstruction and Optimal Control for Nonholonomic Mechanical Systems with Symmetry, PhD thesis, University of California, Berkeley 1997.

[21] W-S. Koon, J.E. Marsden: Optimal control for holonomic and nonholonomic mechanical systems with symmetry and Lagrangian reduction, SIAM J. Control Optim. 35 (1997) 901929 .

[22] P.S. Krishnaprasad: Optimal control and Poisson reduction, Institute for System Research Technical Report 93-87 (1994) 16 pages.

[23] M. de León, J.C. Marrero, E. Martínez: Lagrangian submanifolds and dynamics on Lie algebroids, J. Phys. A: Math. Gen. 38 (2005) R241-R308.

[24] M. de León, P.R. Rodrigues: Methods of Differential Geometry in Analytical Mechanics, North-Holland Math. Ser., 152. Amsterdam, 1989.

[25] K.C.H. Mackenzie: General theory of Lie groupoids and Lie algebroids, London Mathematical Society Lecture Note Series, 213. Cambridge University Press, Cambridge, 2005.

[26] K.C.H. Mackenzie, P. Xu: Lie bialgebroids and Poisson grupoids, Duke Math. J. 73 (1994), 415-452.

[27] E. Martínez: Lagrangian Mechanics on Lie Algebroids, Acta Appl. Math. 67 (2001), 295-320.

[28] E. Martínez: Geometric formulation of Mechanics on Lie algebroids, In Proceedings of the VIII Fall Workshop on Geometry and Physics, Medina del Campo, 1999, Publicaciones de la RSME, 2 (2001), 209-222.

[29] E. Martínez: Reduction in optimal control theory. Rep. Math. Phys. 53 (1) (2004), 79-90.

[30] E. Martínez: Variational calculus on Lie algebroids, preprint (2006), arXiv:math-ph/0603028 To appear in ESAIM (2007).

[31] R. Montgomery: A Tour of Subriemannian Geometries, Their Geodesics and Applications, Mathematical Surveys and Monographs, 91, Americal Mathematical Society, 2002.

[32] R. Skinner, R. Rusk: Generalized Hamiltonian dynamics. I. Formulation on $T^{*} Q \oplus T Q, J$. Math. Phys., 24 (11) (1983), 2589-2594.

[33] R. Skinner, R. Rusk: Generalized Hamiltonian dynamics. II. Gauge transformations, J. Math. Phys., 24 (11) (1983), 2595-2601.

[34] A. Weinstein: Lagrangian Mechanics and groupoids, Fields Inst. Comm. 7 (1996), 207-231.

D. Iglesias: Instituto de Matemáticas y Física Fundamental, Consejo Superior de Investigaciones Científicas, Serrano 123, 28006 Madrid, Spain

E-mail address: iglesias@imaff.cfmac.csic.es

J. C. Marrero: Departamento de Matemática Fundamental, Facultad de Matemáticas, Universidad de la Laguna, La Laguna, Tenerife, Canary Islands, Spain

E-mail address: jcmarrer@ull.es

D. Martín de Diego: Instituto de Matemáticas y Física Fundamental, Consejo SupeRior De Investigaciones Científicas, Serrano 123, 28006 Madrid, Spain

E-mail address: d.martin@imaff.cfmac.csic.es

D. Sosa: Departamento de Economía Aplicada, Facultad de CC. EE. y Empresariales, Universidad de La Laguna, La Laguna, Tenerife, Canary Islands, Spain

E-mail address: dnsosa@ull.es 